ACOUSTIC SOURCE LOCALIZATION EXPLORING THEORY AND PRACTICE

Jelmer Wind 
Dit poefschrift is mede tot stand gekomen door financiële bijdrage van Stichting Technische Wetenschappen (STW).

De promotiecommissie is als volgt samengesteld

Voorzitter en secretaris:

Prof.dr.ir. F. Eising Universiteit Twente

Promotor:

Prof.dr.ir. A. de Boer

Universiteit Twente

Assistent promotor:

Dr. ir. M.H.M. Ellenbroek

Universiteit Twente

Leden:

Dr.ir. A.P. Berkhoff

Universiteit Twente

Prof.dr.ir. A. Gisolf

Technische Universiteit Delft

Prof.dr.ir. E.W.C. van Groesen

Univeristeit Twente

Prof.dr.ir. H.W.M. Hoeijmakers

Universiteit Twente

Dr.ir. I. Lopez Arteaga

Technische Universiteit Eindhoven

Deskundige en reserve:

Prof.dr.ir. W.F. Druyvesteyn

Universiteit Twente

J.W. Wind Acoustic Source Localization, Exploring Theory and Practice. PhD Thesis, University of Twente, Enschede, The Netherlands, 2009. 


\title{
ACOUSTIC SOURCE LOCALIZATION EXPLORING THEORY AND PRACTICE
}

\author{
PROEFSCHRIFT
}

ter verkrijging van

de graad van doctor aan de Universiteit Twente, op gezag van de rector magnificus,

Prof.dr. H. Brinksma,

volgens het besluit van het College voor Promoties

in het openbaar te verdedigen

op woensdag 18 november 2009 om 16.30 uur

door

Jelmer Wilco Wind

Geboren op 14 december 1979

te Zwolle 
Dit proefschrift is goedgekeurd door de promotor

Prof.dr.ir. A. de Boer

en de assistent promotor

Dr.ir. M.H.M. Ellenbroek 


\section{Summary}

Over the past few decades, noise pollution became an important issue in modern society. This has led to an increased effort in the industry to reduce noise. Acoustic source localization methods determine the location and strength of the vibrations which are the cause of sound based on measurements of the sound field.

This thesis describes a theoretical study of many facets of the acoustic source localization problem as well as the development, implementation and validation of new source localization methods. The main objective is to increase the range of applications of inverse acoustics and to develop accurate and computationally efficient methods for each of these applications. Four applications are considered.

Firstly, the inverse acoustic problem is considered where the source and the measurement points are located on two parallel planes. A new fast method to solve this problem is developed and it is compared to the existing method planar nearfield acoustic holography (PNAH) from a theoretical point of view, as well as by means of simulations and experiments. Both methods are fast but the new method yields more robust and accurate results.

Secondly, measurements in inverse acoustics are often point-by-point or full array measurements. However a straightforward and cost-effective alternative to these approaches is a sensor or array which moves through the sound field during the measurement to gather sound field information. The same numerical techniques make it possible to apply inverse acoustics to the case where the source moves and the sensors are fixed in space. It is shown that the inverse methods such as the inverse boundary element method (IBEM) can be applied to this problem. To arrive at an accurate representation of the sound field, an optimized signal processing method is applied and it is shown experimentally that this method leads to accurate results.

Thirdly, a theoretical framework is established for the inverse acoustical problem where the sound field and the source are represented by a cross-spectral matrix. This problem is important in inverse acoustics because it occurs in the inverse calculation of sound intensity. The existing methods for this problem are analyzed from a theoretical point of view using this framework and a new method is derived 
from it. A simulation study indicates that the new method improves the results by $30 \%$ in some cases and the results are similar otherwise.

Finally, the localization of point sources in the acoustic near field is considered. MUltiple SIgnal Classification (MUSIC) is newly applied to the Boundary element method (BEM) for this purpose. It is shown that this approach makes it possible to localize point sources accurately even if the noise level is extremely high or if the number of sensors is low. 


\section{Samenvatting}

Geluidsoverlast is de laatste decennia een belangrijk maatschappelijk probleem geworden. Daarom wordt er in de industrie steeds meer aandacht besteed aan het verminderen van geluid. Akoestische bronlokalisatiemethoden bepalen de locatie en sterkte van de trillingen die het geluid veroorzaken, op basis van metingen van het geluidsveld.

Dit proefschrift beschrijft een theoretische studie van de vele facetten van het akoestische bronlokalisatieprobleem en ook de ontwikkeling, implementatie en validatie van nieuwe bronlokalisatiemethoden. Het hoofddoel is het verbreden van de toepassingsmogelijkheden van bronlokalisatiemethoden en het ontwikkelen van nauwkeurige en efficiënte methoden voor de nieuwe toepassingen. Er worden vier toepassingen beschouwd.

Ten eerste wordt het probleem beschouwd waar de bron en de meetpunten op twee parallelle vlakken liggen. Een nieuwe, snelle methode wordt ontwikkeld en vergeleken met de bestaande methode PNAH. Deze vergelijking vindt plaats door middel van theorie, simulaties en experimenten. Beide methoden zijn snel maar de nieuwe methode levert meer betrouwbare en nauwkeurige resultaten.

Ten tweede, metingen in de inverse akoestiek worden vaak uitgevoerd als puntvoor-punt metingen of als volledige arraymetingen. Een eenvoudig en goedkoop alternatief is een sensor of array die tijdens de meting door het geluidsveld beweegt om data over het geluidsveld te verzamelen. De numerieke technieken die voor dit probleem geschikt zijn kunnen ook worden gebruikt voor meetopstellingen waar de bron beweegt en de sensoren stilstaan. In dit proefschrift wordt aangetoond dat inverse methoden zoals de inverse randelementenmethode (IBEM) kan worden toegepast voor dit probleem. Om te komen tot een nauwkeurige beschrijving van het geluidsveld wordt een geoptimaliseerde singaalbewerkingstechniek toegepast. Met behulp van experimenten wordt aangetoond dat deze signaalbewerkingsaanpak tot nauwkeurige resultaten leidt.

Ten derde wordt een theoretisch kader ontwikkeld voor het inverse akoestische probleem waar het geluidsveld en de bron worden uitgedrukt als een kruisspectrummatrix. Dit probleem is belangrijk in de inverse akoestiek omdat het voorkomt 
bij het inverse berekenen van de geluidsintensiteit. De bestaande methoden voor dit probleem worden theoretisch geanalyseerd met behulp van dit kader en een nieuwe methode wordt aan de hand van dit kader afgeleid. Een simulatiestudie toont aan dat de nieuwe methode de resultaten in sommige gevallen $30 \%$ verbetert en dat de resultaten in de andere gevallen vergelijkbaar zijn.

Als laatste wordt de lokalisatie van puntbronnen in het nabijheidsveld van de bron beschouwd. De MUSIC methode wordt toegepast op de randelementenmethode (BEM). Deze nieuwe aanpak maakt het mogelijk om zelfs puntbronnen te lokaliseren als het ruisniveau extreem hoog of het aantal sensoren laag is. 


\section{Contents}

1 Introduction 1

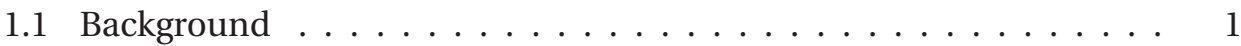

1.2 The physics of sound $\ldots \ldots \ldots \ldots \ldots \ldots \ldots \ldots$

1.3 Inverse acoustic problems . . . . . . . . . . . . . . . 2

1.4 Direct measurement of acoustic sources . . . . . . . . . . . 4

1.5 Previous research in inverse acoustics . . . . . . . . . . . 6

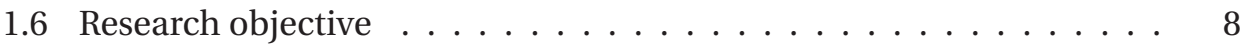

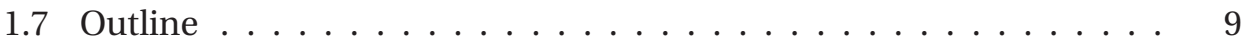

2 Acoustic modeling 11

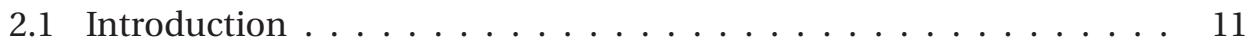

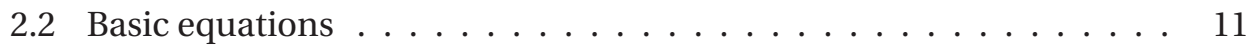

2.3 The Helmholtz integral equation . . . . . . . . . . . . . . . . 13

2.4 Fourier acoustics . . . . . . . . . . . . . . . . . 15

2.5 Numerical acoustic modeling . . . . . . . . . . . . . . . 17

2.6 Summary . . . . . . . . . . . . . . . . . . . 19

3 Regularization 21

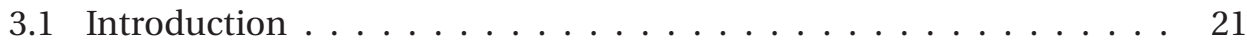

3.2 The SVD applied to regularization problems . . . . . . . . . . . 22

3.3 Alternative SVDs . . . . . . . . . . . . . . . . . . . . . . . 24

3.4 Examples . . . . . . . . . . . . . . . . . . . . 27

3.5 Numerical implementation . . . . . . . . . . . . . . . . . . . . . . . . . . 32

3.6 Summary ....................... 36

4 Planar inverse acoustics $\quad 37$

4.1 Introduction . . . . . . . . . . . . . . . 37

4.2 Planar nearfield acoustic holography . . . . . . . . . . . . . 38

4.3 Toeplitz Rayleigh integral method . . . . . . . . . . . . . . . . 46

4.4 Comparative study . . . . . . . . . . . . . . . . . . . . 52 


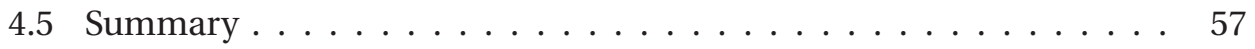

5 Statistical signal processing applied to moving sensors 61

5.1 Introduction . . . . . . . . . . . . . . . . . 61

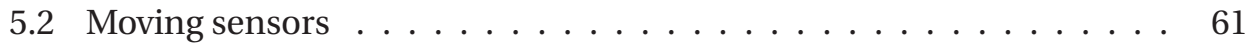

5.3 Random vibrations . . . . . . . . . . . . . . . . . . . . 66

5.4 Fourier analysis . . . . . . . . . . . . . . . . . 71

5.5 Transfer function estimation . . . . . . . . . . . . . . . 79

5.6 Experimental validation using moving sensors . . . . . . . . . . . 82

5.7 Summary . . . . . . . . . . . . . . . . . . 87

6 Inverse acoustics using cross-spectral matrices $\quad 89$

6.1 Introduction . . . . . . . . . . . . . . . . . . . . . 89

6.2 Cross-spectral modeling . . . . . . . . . . . . . . . . . . 90

6.3 Regularization . . . . . . . . . . . . . . . . . . . . 95

6.4 Case study . . . . . . . . . . . . . . . . . . . . . . . . 98

6.5 Point-to-surface cross spectra . . . . . . . . . . . . . . . . . . 104

6.6 DAMAS . . . . . . . . . . . . . . . . . . . 106

6.7 Rank of the calculated cross-spectral matrix . . . . . . . . . . . 107

6.8 Summary . . . . . . . . . . . . . . . . . . . . 108

7 Two BEM-based point-source localization methods 109

7.1 Introduction . . . . . . . . . . . . . . . . . . . . 109

7.2 MUSIC . . . . . . . . . . . . . . . . . . . 109

7.3 A least squares approach . . . . . . . . . . . . . . . . . . . 112

7.4 Relation to regularization . . . . . . . . . . . . . . . . 113

7.5 Case study . . . . . . . . . . . . . . . . . . . . . . . . . . . . . 114

7.6 Summary . . . . . . . . . . . . . . . . . . . 114

8 Conclusions and Recommendations 119

8.1 Conclusions . . . . . . . . . . . . . . . . . . . . . . . 119

8.2 Recommendations . . . . . . . . . . . . . . . 120

A An exact description of the sound at the field points 123

B Total least squares $\quad 127$

C Rank of the source matrix 129

D DAMAS 133

$\begin{array}{ll}\text { Bibliography } & 134\end{array}$ 




\section{Chapter 1}

\section{Introduction}

\subsection{Background}

Sound plays an important role in our daily lives. It helps us make sense of our environment, warns us of approaching danger and - from person to person - it carries our communication.

In modern society, technical devices such as personal audio systems, cars and computers make sound that we hear almost everywhere. There is an increasing tendency in the industry to improve the sound quality of their products. Often, the goal is to make the sound softer or less unpleasant, but sometimes, the goal is to convey a positive product identity. For example, a sports car should sound tough, a quality saloon car should sound luxurious and the computerized voice of a GPS system should be natural and comprehensible. To make it possible to improve the sound quality of a product, a solid understanding of the causes of sound is essential.

To gain insight in these causes of sound, this thesis focuses on methods to calculate the location and strength of the sound sources by combining experimental data with theoretical knowledge of the behavior of sound.

\subsection{The physics of sound}

The sensation of sound is caused by varying pressures in the ear. This pressure variation, which is called the sound pressure, is much smaller than the constant ambient pressure and travels through the medium (air) in the form of waves.

The human ear is impressively sensitive to these pressure variations. The softest audible tone of $1 \mathrm{kHz}$ has a sound pressure of $20 \mu \mathrm{Pa}(0 \mathrm{~dB})$ whereas the threshold of pain is as much as $20 \mathrm{~Pa}(120 \mathrm{~dB})$. Although this loud tone has a million times the 
amplitude of the threshold of hearing, it is still a small fraction of the ambient pressure, which is $10^{5} \mathrm{~Pa}$. The audible frequency range varies from one individual to the next. For individuals with good hearing, audible sound is between $20 \mathrm{~Hz}$ and $20 \mathrm{kHz}$, spanning 10 octaves.

There are many physical processes that can cause sound. A rough classification of sound sources is as follows. Firstly, Structure induced sound is sound caused by mechanical vibrations such as the sound generated by a loudspeaker. Secondly, Flow induced sound is sound induced by a disturbed flow. An example is noise of a turbulent flow around an aircraft. Thirdly, Thermal induced sound is sound caused by local variations in the temperature of the fluid. An example is lightning, where the electric discharge produces a large change in the temperature, causing a sudden expansion of the air.

Acoustic theory describes the propagation of sound through air or any other medium. In an acoustic model, the sources are often characterized by the velocity at all points of a surface. This boundary condition fully determines the sound field.

\subsection{Inverse acoustic problems}

A distinction can be made between forward acoustic problems and inverse acoustic problems (see figure 1.1). In a forward acoustic problem, a complete description of the acoustic sources of sound is known and the solution process requires finding a description of the sound field. An example is the problem of calculating the sound field caused by a loudspeaker based on known velocities at the surface of the loudspeaker. In the inverse problem, the sound field is known and the acoustic sources must be calculated. A typical inverse acoustic method aims to find the velocities or pressures at the source surface based on sound field measurements near the source

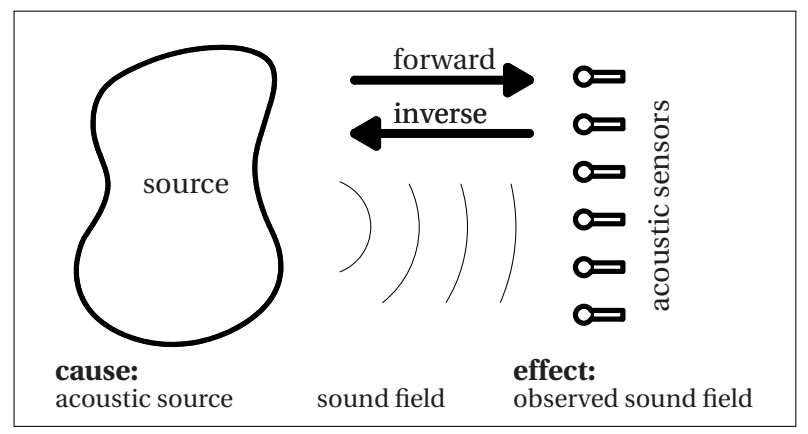

Figure 1.1: Illustration of the forward and inverse problem (image from Visser [89]). 


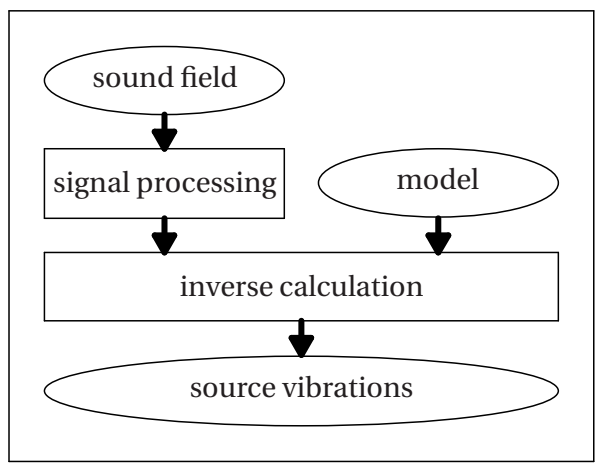

Figure 1.2: The steps required in inverse acoustics
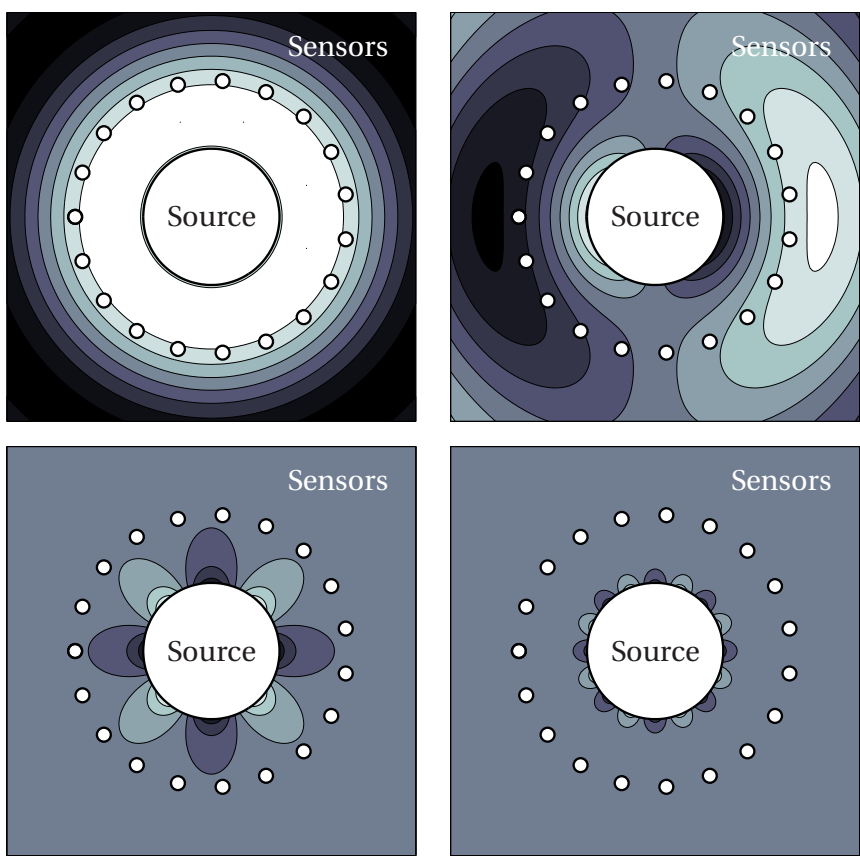

Figure 1.3: The pressure field caused by a circular source in 2D which exhibits source vibrations that are smooth (top) and oscillatory (bottom). The real part is depicted. White $(+)$ and Black (-) are scaled to the extremal values of the pressure field. 
surface. The main focus of this study is on the inverse problem.

Inverse acoustic methods consist of the following steps (see figure 1.2). The acoustic pressure or particle velocity of the sound field is measured at a large number of field points close to the source surface. Signal processing techniques are applied to the measurement data to represent the sound field as statistical quantities such as cross spectra in the frequency domain. Furthermore, a forward model is determined. This model describes the relation between the source vibrations and the field data at some frequency and it is represented by a system of equations. To arrive at an approximation of the source vibrations, an inverse calculation is performed.

The inverse calculation is a discrete ill-posed problem. From an algebraical point of view, the system of equations which must be solved to calculate the source vibrations is inherently ill-conditioned. Hence, the direct solution of the system yields unphysical results if measurement noise is present. Contrary to this algebraical point of view, the problem can also be considered from a physical point of view. The source vibration consists of both smooth and oscillatory components. Smooth components decay slowly with the distance to the source and oscillatory components decay fast (see figure 1.3). If the inverse problem is solved directly, the oscillatory components are multiplied by a large factor to reverse their attenuation, causing any noise to be multiplied by the same factor. This causes the calculated source vibrations to be overshadowed by noise. It is impossible to determine the strength of the highly oscillatory components from the available measurement data because their contribution is overshadowed by noise. However, it is possible to calculate the strength of the smooth components. To find a meaningful approximation of the source vibration, the amplitude and phase of the source components which decay slowly enough are calculated and the the other components are set to zero. This is the essence of regularization of inverse problems. It is discussed in chapter 3.

An alternative way to localize acoustic sources is to perform measurements at the source, instead of close to the source. This is discussed in the next section. Inverse acoustic methods have the ability to localize sources even if the direct measurement methods do not apply. Successful applications of inverse acoustic methods include tire-road noise, car interior noise, various applications in aviation and consumer products such as a hairdryer.

\subsection{Direct measurement of acoustic sources}

An important disadvantage of inverse acoustic methods is the fact that the accuracy which can be achieved does not only depend on the noise level of the sen- 
sors but also on the unknown source vibration. Furthermore, a good agreement between model and experiment is necessary to arrive at accurate results. These inherent disadvantages of inverse acoustic methods are avoided by measuring the acoustic sources directly, such that the inverse acoustic problem does not need to be solved. This section briefly summarizes the advantages and disadvantages of a few direct measurement methods.

For structure induced sound, it is sufficient to measure the vibration of the source surface itself. A few methods to identify structural sources are as follows [16].

- Accelerometers are sensors which can be fixed to the structure surface to measure its acceleration. An advantage of this approach is its comparatively low cost. A disadvantage is the fact that mass is added to the structure.

- Laser Doppler Velocimeters are devices which are capable of measuring the instantaneous velocity of the surface of a structure. The velocity is measured by directing a beam of laser light at the target point and measuring the Doppler-shifted wavelength of the reflected light which is returned from the moving surface, using an interferometer. A disadvantage of this approach is the requirement to measure a clear reflection from the laser. An important advantage is that the measurement location can be controlled by positioning the laser device, or by means of positioning mirrors.

- Acoustic particle velocity sensors can be placed so close to the source that there is a negligible difference between the velocity of the structure and the acoustic particle velocity. These measurements are known as very nearfield particle velocity measurements $[7,65,6,101]$. This approach is comparatively new and less accepted in the scientific community than the other two approaches. Disadvantages of this approach are the fact that the sensors must be placed very close to the surface the placement of a sensor in the very near field of a source can change the sound field in some applications. For example, a particle velocity sensor placed in the flow of a wind tunnel also measures the turbulent flow which the sensor causes. An advantage is the fact that the method is not limited exclusively to structure induced sound.

Measurements of the structure surface are insufficient if the sources are aeroacoustic, thermal or a combination of the three basic types. A non-intrusive alternative is laser photon correlation spectroscopy, which measures the acoustic flows at the location where two laser beams coincide [65]. Due to the extreme technical complexity of this technique, its use has been limited mainly to the academical world until the current time. 
In summary, direct measurement techniques are attractive because they do not require an inverse calculation. Inverse acoustic methods are attractive if the direct measurement methods are expensive, inapplicable or difficult to use.

\subsection{Previous research in inverse acoustics}

Inverse acoustic methods can be roughly divided into two classes: farfield methods and nearfield methods.

The far-field methods are widely used in aeroacoustic studies. Before the mid 1980s, the processing of array microphone signals for aeroacoustic studies involved time delay shifting of signals and summing in order to strengthen their contribution from, and thus 'focus' on, chosen locations over the surfaces or positions in the flow field [8]. Over the years, with great advances in computers, this basic 'delay and sum' processing has gained many descendants, some of which are known as beamforming methods. Many of these methods are only applicable if the distance between the source and the sensors is more than a few wavelengths. Hence, the term far-field methods is used.

A more accurate characterization of the source vibration can be achieved if the sensors are placed close to the source. Near-field methods are necessary to model and solve the inverse problem in this case. In 1985, Maynard, Williams and Veronesi proposed a method known as nearfield acoustic holography (NAH) based on early research by Graham $[45,85]$. Contrary to earlier approaches in acoustic holography, their model accurately describes the sound field close to the source. This model is used in the inverse calculation, making it possible to visualize features of the source which are smaller than the acoustic wavelength, if the sensors are placed close to the source. The main focus of their work lies of the planar inverse acoustic problem, where the source and field are parallel planes and their method is termed Planar Nearfield Acoustic Holography (PNAH). In 2001, Steiner and Hald proposed an alternative to PNAH which is known as statistically optimized nearfield acoustical holography (SONAH) [79]. The goal of their study was to achieve a higher accuracy by using a model which is not based on the discrete Fourier transform (see also chapter 4). The limitation in geometries was removed in 1989, when Maynard and Veronesi proposed the inverse boundary element method (IBEM). The boundary element method (BEM) is used to arrive at a forward model and this model is subsequently inverted using numerical techniques [86].

The main focus of the current thesis lies on inverse BEM (IBEM) but many of the principles can be applied to other methods. Three noteworthy alternatives are HELS, ESM and IPTF. The Helmholtz equation-least-squares (HELS) method ap- 
plies an inverse acoustic method for spherical sources to sources which are not necessarily spherical. This method was proposed by Wang in the mid 1990s [92]. A similar method is termed Equivalent source method (ESM) and aims to represent the sound field by a number of monopole sources located at the source. This equivalent source representation of the source is then used to calculate the sound field at points where no measurements are performed. The method was proposed by Sarkissian in 2004 [70, 71]. The third method is termed the inverse patch transfer function (IPTF) method. It uses an acoustic finite element model of a finite volume, completely enclosed by the source and the field points. By measuring both pressure and particle velocity at the field points, the source velocity and pressure can be calculated without the need for a model of the sound outside the finite volume enclosed by the source and the field points. The method was proposed in 2008 by Totaro et al. [83].

The advances in inverse modeling have been accompanied by great advances in measurement and signal processing. The early experimental setups made use of a full array of microphones to measure the sound field at all field points simultaneously. To increase the number of field points at a low cost, Hald introduced the concept of a reference sensor in 1989, making point-by-point measurements a viable alternative to full array measurements $[22,48]$. Furthermore, acoustic particle velocity sensors have been introduced to inverse acoustics as an alternative to pressure microphones. In 2002, Visser showed by means of simulations that measurements of the velocity normal to the surface lead to more accurate results than measurements of the pressure $[87,88]$. The problem was later studied by Jacobsen and co-workers [35, 34].

The mathematical study of inverse problems started long before the 1980s. During the beginning of the 20th century, Hadamard defined the concept of well-posed and ill-posed problems. In essence, a problem is defined to be ill-posed if the solution is not unique or if an arbitrarily small perturbation of the data can cause an arbitrarily large perturbation in the solution [28]. Ill-posed problems were ignored for the most part until the 1940s and 1950s at which time Tikhonov began his investigation. His study lead to the famous Tikhonov regularization method. The use of the Singular Value Decomposition in inverse problems goes back to Hanson [31] who, in 1971, proposed the Truncated Singular Value Decomposition (TSVD). From the 1980s to the present day, the study of inverse problems has been one of the fastest growing areas in applied mathematics. Many algorithms have been proposed and different types of inverse problems and their solutions have been studied.

A more fundamental aspect of the mathematical research on inverse problems is the study of regularization itself. Some researchers study the convergence rate of the inverse solution as the noise tends to zero [21]. Since the optimal convergence 
rate is achieved by many different methods, the results of this field do not help to choose a suitable regularization method. Other researchers approach the regularization problem from a statistical point of view (see for example Tarantola [80]). A weakness of this approach is the fact that it relies on assumed probabilities which cannot be validated (the so-called prior probability density function). Tikhonov regularization follows from this statistical framework under certain assumptions and definitions.

Given this summary of the literature, it is concluded that since the 1980s inverse acoustics has progressed to a large field of engineering science. Many ideas and methods have been proposed and applied to engineering problems in acoustic source localization.

\subsection{Research objective}

This study takes place within the project Inverse Acoustics (TWO 6618) of the Dutch Technology Foundation (STW). The objectives of the project are outlined in the following section of the project proposal [69].

"Current methods for source localization are seriously hampered by the fact that they are costly with respect to hardware and computational effort and - even more important - they can only be applied to a limited class of problems. Moreover, closely spaced sources, as frequently encountered in practice, cannot be distinguished. To overcome these shortcomings a new project is proposed in which three, closely coordinated lines of improvement are sought.

(1) improved numerical modeling and validation,

(2) improved measurement techniques and signal processing,

(3) fast and user-friendly data acquisition and data reduction methods."

The research on the topic improved measurement techniques and signal processing has been carried out by the Dynamics and Control group of the Eindhoven University of Technology (D\&A-TU/e) and it has focused on the improvement of PNAH [73]. The developed methods are compared to various other methods in chapter 4.

The topic fast and user-friendly data acquisition and data reduction methods has been carried out by the Signals and Systems group of the University of Twente. It has consisted of the development, production and validation of parallel hardware to process measurement data from a microphone array. The inverse techniques of chapters 6 and 7 can be applied directly to the new hardware.

The current study considers the topic improved numerical modeling and validation. Given the goals outlined in the project proposal, the general goals of this 
line of research are

- the development of efficient computational methods,

- the improvement of the accuracy.

The central theme of this thesis is the development and validation of computational methods for four different topics in inverse acoustics. To develop accurate and efficient methods for each of these topics, it is necessary to gain a deeper understanding of them. For this purpose, insights from acoustics and applied mathematics are combined. The following topics are considered in detail: planar inverse acoustics, the use of moving sensors, inverse acoustics using cross-spectral matrices and point-source localization methods.

\subsection{Outline}

This chapter has given an overview of the field of inverse acoustics and this introduction is continued in chapters 2 and 3 . Chapter 2 presents a brief review of the acoustic theory and the methods to solve forward acoustic problems. In chapter 3 , a survey of SVD-based regularization methods and its generalizations is given. The emphasis lies on the application of regularization to the boundary element method (BEM).

The planar inverse acoustic problem is studied in chapter 4 . A theoretical and practical comparison between PNAH and SONAH is discussed. Furthermore, a new fast algorithm to solve the inverse acoustic problem of SONAH is presented.

The problem of moving sensors in inverse acoustics is considered in chapter 5 . The basics of statistical signal processing and nonparametric spectral estimation are introduced and it is shown that conventional spectral estimation techniques do not give satisfactory results. To solve this problem, the use of transfer functions and the multi-taper method is proposed. It is shown that this approach leads to accurate results.

The inverse acoustic problem where the source and the field are represented by cross-spectral matrices is studied in chapter 6 . An important application of this problem is the inverse calculation of sound intensity. A theoretical framework is presented in which the existing methods for this purpose have a place. Furthermore, a new method follows naturally from this framework. The new and existing methods are compared and it is shown that the DAMAS method in aeroacoustics can be derived from the same framework.

The localization of acoustic point sources is considered in chapter 7. The MUSIC method, which is widely used to identify the direction of arrival of plane waves, 
is applied to BEM. In this new application, the MUSIC method can acurately localize multiple point sources on a surface close to the source, using arbitrary geometries. The method is compared to a straightforward least squares method.

Finally, chapter 8 presents the conclusions and recommendations of this study. 


\section{Chapter 2}

\section{Acoustic modeling}

\subsection{Introduction}

This chapter introduces the basic equations of acoustics and discusses a number of methods to perform acoustic calculations on a computer. Based on these computer models, the inverse solution can be calculated using techniques discussed in chapter 3 . For a more extensive introduction to the theory of acoustics, the reader is referred to the textbooks $[5,38,59]$.

The outline of this chapter is as follows. Section 2.2 introduces the basic equations and section 2.3 considers the Helmholtz integral equation. Section 2.4 considers the case where the source is an infinite plane and explains the application of the 2D spatial Fourier transform to solve the acoustic equations in the wavenumber domain. Discretization in the spatial domain is considered in section 2.5. This spatial discretization forms the foundation of the boundary element method, which is used throughout this study. A summary is given in section 2.6.

\subsection{Basic equations}

Lord Rayleigh was among the first to mathematically formulate the principle of sound propagation. In 1877, he published his major work on acoustics The Theory of Sound $[67,68]$. One of the most important results is the acoustic wave equation which describes acoustic wave propagation of a fluid at rest. It is given by

$$
\nabla^{2} \hat{p}+\frac{1}{c_{0}} \frac{\partial^{2} \hat{p}}{\partial t^{2}}=0
$$

where $\hat{p}(\vec{x}, t)$ and $c_{0}$ are the acoustic pressure and the speed of sound respectively. The vector $\vec{x}$ denotes an arbitrary point in the fluid. Furthermore, the accent ${ }^{\wedge}$ is 
used for functions of time and it is removed for functions of the angular frequency. To keep the notation brief, the function $\hat{p}(\vec{x}, t)$ is denoted as $\hat{p}(\vec{x})$ or simply $\hat{p}$ where it improves the clarity.

The acoustic equations are represented in the frequency domain by means of the following definition of the Fourier transform

$$
p(\omega, \vec{x})=\int_{-\infty}^{\infty} \hat{p}(t, \vec{x}) e^{-\mathrm{i} \omega t} d t
$$

where i and $\omega$ are the imaginary unit and the angular frequency respectively. Furthermore, $p(\vec{x}, \omega)$ is the Fourier transform of $\hat{p}(\vec{x}, t)$.

The frequency domain representation of the wave equation is the Helmholtz differential equation

$$
\nabla^{2} p+k^{2} p=0
$$

where $k=\omega / c_{0}$ is the acoustic wave number. The acoustic fluid velocity is related to the acoustic pressure by Euler's equation of motion. In the frequency domain, it is given by

$$
-\mathrm{i} \omega \rho_{0} \vec{v}=\nabla p
$$

where $\rho_{0}$ and $\vec{v}(\vec{x}, \omega)$ are the density of the fluid at rest and the Fourier transform of the particle velocity vector respectively.

The acoustic equations even apply if the size of the acoustic domain is unbounded but it is necessary to add the assumption that no sound arrives from infinity. This condition, which is essentially the assumption that all sources lie within a bounded region, leads to the Sommerfeld radiation condition [59].

$$
\lim _{r \rightarrow \infty} r\left(\frac{\partial p}{\partial r}+i k p\right)=0
$$

where $r$ is the radius in a fixed spherical coordinate system.

The sound intensity is the third and last acoustic quantity that will be introduced. It is the acoustic power flux per unit of area, which is a vectorial quantity indicating the direction of the power flow. The instantaneous sound intensity $\vec{I}$ is given by [17]

$$
\overrightarrow{\hat{I}}(t)=\hat{p}(t) \overrightarrow{\hat{v}}(t)
$$

The time average of the instantaneous sound intensity is termed the mean intensity, which is called the active intensity in the frequency domain. For harmonic 
sound waves the active sound intensity is ${ }^{1}$

$$
\vec{I}_{a}=\frac{1}{2} \operatorname{Re}(P \overrightarrow{\vec{V}}) \quad \text { with } \quad\left\{\begin{array}{l}
\hat{p}(t)=\operatorname{Re}\left(P e^{\mathrm{i} \omega t}\right) \\
\hat{\vec{v}}(t)=\operatorname{Re}\left(\vec{V} e^{\mathrm{i} \omega t}\right)
\end{array}\right.
$$

where $P$ and $\vec{V}$ are complex constants and Re and $\overline{(\cdot)}$ denotes the real part and the complex conjugate respectively. The reactive sound intensity is half the imaginary part of $P \overrightarrow{\vec{V}}$ [33]. Sound intensity is purely active in propagating waves and purely reactive in standing waves. The complex sound intensity $\frac{1}{2} P \overrightarrow{\vec{V}}$ contains both the real and imaginary parts.

This study considers broadband sound in the frequency domain, contrary to harmonic waves. Depending on the definition of the Fourier transform, the resulting expression of the sound intensity can change by a scalar constant. To conform to other literature on the subject, the active sound intensity $I_{A}$ for broadband sound is defined here to be

$$
\vec{I}_{A}(\omega)=\frac{1}{2} \operatorname{Re}(p(\omega) \overline{\vec{v}(\omega)})
$$

\subsection{The Helmholtz integral equation}

To find a solution of the acoustic equations, the source is often modeled to be a vibrating surface which has known surface velocities or pressures. Although the sound field can be calculated directly using the Helmholtz differential equation, a useful alternative is the Helmholtz integral equation (HIE) which forms the foundation of the boundary element method (BEM). The HIE is briefly discussed in this section and more information can be found in [59].

The Helmholtz integral equation relates the pressure at an arbitrary point $p(\vec{x})$ to the pressure $p(\vec{y})$ and the normal velocity $v_{n}(\vec{y})$ at a closed surface surface $S$, such that $\vec{y} \in S$. This surface can be the boundary of an object or any other surface in space, provided that all sources lie in the interior of $S$. The Helmholtz integral equation is given by (see also figure 2.1)

$$
\alpha(\vec{x}) p(\vec{x})=\oint_{S}\left(\frac{\partial G(|\vec{y}-\vec{x}|)}{\partial \vec{n}(\vec{y})} p(\vec{y})+\mathrm{i} \omega \rho_{0} G(|\vec{y}-\vec{x}|) v_{n}(\vec{y})\right) d S
$$

Where $\alpha$ is a space angle, which equals one if $\vec{x}$ is located in the acoustic field and it is smaller than one if $\vec{x}$ is located at the surface. The function $G$ is Green's function.

\footnotetext{
${ }^{1}$ The real part of the expressions $p(t)$ and $v(t)$ has been taken to correct an omission in the book Sound intensity by FJ. Fahy (Section 4.5 page 48, line 5-7) [17].
} 


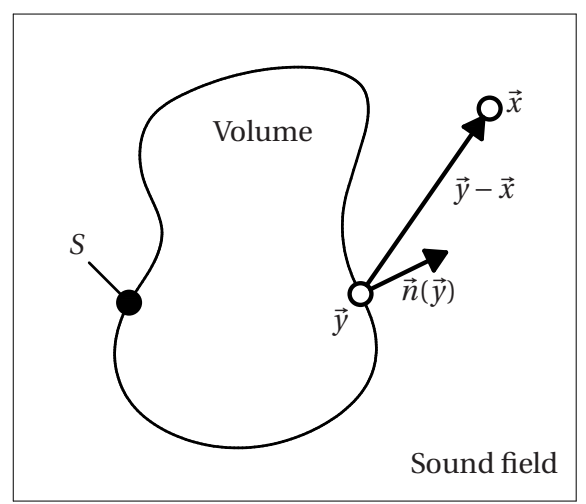

Figure 2.1: Nomenclature in the Helmholtz integral equation in accordance with equation 2.9

It is the response of the inhomogenious Helmholtz differential equation to a Dirac delta distribution in space that also satisfies the Sommerfeld radiation condition. In three-dimensional space, it is given by the following equation.

$$
G(|\vec{y}-\vec{x}|)=\frac{e^{-\mathrm{i} k|\vec{y}-\vec{x}|}}{4 \pi|\vec{y}-\vec{x}|}
$$

The notation $\frac{\partial G}{\partial \vec{n}}$ should be interpreted as follows.

$$
\frac{\partial G(\vec{x})}{\partial \vec{n}(\vec{y})}=\nabla G(\vec{x}) \cdot \vec{n}(\vec{y}) \quad \text { where } \quad \vec{y} \in S
$$

The Helmholtz integral equation can also be derived for the case where the sound field lies inside the surface as well as for scattering problems, where a rigid object is subjected to an incident sound field. Many other cases have also been researched. For more information, the reader is referred to [90] and references therein.

For a flat panel in an infinite baffle, the Helmholtz integral equation reduces to Rayleigh's second integral, also known as the Rayleigh integral [5]. It is given by (see figure 2.2)

$$
p(\vec{x})=\frac{\mathrm{i} \omega \rho_{0}}{2 \pi} \int_{S} v_{n}(\vec{y}) \frac{e^{-\mathrm{i} k|\vec{y}-\vec{x}|}}{|\vec{y}-\vec{x}|} d S
$$

Where $S$ denotes an area on the baffle surface. 


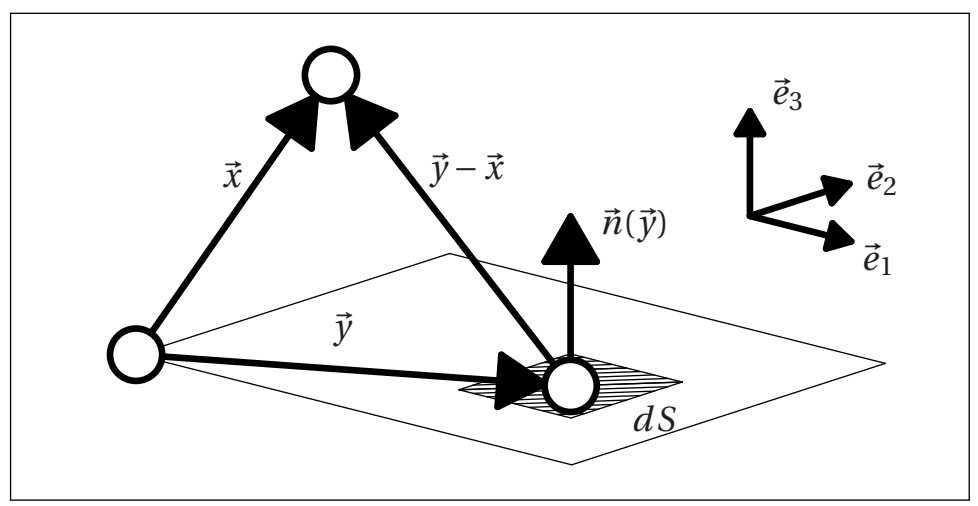

Figure 2.2: Nomenclature in Rayleigh's second integral in accordance with equation 2.12. Coordinate system as used in section 2.4

\subsection{Fourier acoustics}

Planar nearfield acoustic holography (PNAH) is an inverse acoustic method which is based on the 2D spatial Fourier transform of the sound field. Contrary to the methods used in optical holography, it can localize acoustic sources which are smaller than a wavelength because it uses a forward model which describes the evanescent waves, which have a short wavelength and decay exponentially with the distance to the source. As stated by Williams [97], the method was originally proposed by Graham in 1969. It has been further developed by Williams, Maynard and others, starting in 1980 [97, 45]. Similar methods have been developed for cylindrical and spherical coordinate systems and this large body of work was summarized by Williams in 1999 in the reference work Fourier Acoustics [94].

This section explains the forward model of PNAH and the inverse calculation is considered in chapter 4 . The equations derived here give a straightforward description of the relation between the acoustic vibrations at an infinite source plane and an infinite field plane, located some distance $x_{3}$ above the source. To derive the relations, a Cartesian coordinate system $\vec{x}=\left\{x_{1}, x_{2}, x_{3}\right\}^{T}$ is introduced. The Rayleigh integral (equation 2.12) can now be written as follows.

$$
p\left(x_{1}, x_{2}, x_{3}\right)=\frac{\mathrm{i} \omega \rho_{0}}{2 \pi} \cdot \int_{-\infty}^{\infty} \int_{-\infty}^{\infty} v_{n}\left(y_{1}, y_{2}\right) \frac{e^{-\mathrm{i} k} \sqrt{\left(y_{1}-x_{1}\right)^{2}+\left(y_{2}-x_{2}\right)^{2}+x_{3}^{2}}}{\sqrt{\left(y_{1}-x_{1}\right)^{2}+\left(y_{2}-x_{2}\right)^{2}+x_{3}^{2}}} d y_{1} d y_{2}
$$

This equation can be recognized as a 2D spatial convolution. By the convolution theorem, the Fourier transform of a convolution is an ordinary product of the Fourier transforms. The 2D Fourier transform of a function $f\left(x_{1}, x_{2}\right)$ and its inverse 
are defined as follows.

$$
\begin{aligned}
& \tilde{f}\left(k_{1}, k_{2}\right)=\mathscr{F}(f)=\int_{-\infty}^{\infty} \int_{-\infty}^{\infty} f\left(x_{1}, x_{2}\right) e^{-\mathrm{i} k_{1} x_{1}-\mathrm{i} k_{2} x_{2}} d x_{1} d x_{2} \\
& f\left(x_{1}, x_{2}\right)=\mathscr{F}^{-1}(\tilde{f})=\frac{1}{4 \pi^{2}} \int_{-\infty}^{\infty} \int_{-\infty}^{\infty} \tilde{f}\left(k_{1}, k_{2}\right) e^{\mathrm{i} k_{1} x_{1}+\mathrm{i} k_{2} x_{2}} d k_{1} d k_{2}
\end{aligned}
$$

The Fourier transform of a function of the source coordinates $f\left(y_{1}, y_{2}\right)$ is defined in the same way. It can be shown that the Fourier transform of Green's kernel (equation 2.10) has the following analytical form [94].

$$
\begin{aligned}
\mathscr{F}\left(\frac{\mathrm{i} \rho_{0}}{2 \pi} \frac{e^{-\mathrm{i} k} \sqrt{x_{1}^{2}+x_{2}^{2}+x_{3}^{2}}}{\sqrt{x_{1}^{2}+x_{2}^{2}+x_{3}^{2}}}\right)=\overline{\rho_{0} c_{0} \frac{k}{k_{3}} e^{\mathrm{i} k_{3} x_{3}}} & \text { where } \\
& k_{3}=\sqrt{k^{2}-k_{1}^{2}-k_{2}^{2}}
\end{aligned}
$$

This expression is the complex conjugate of the expression in referred book [94] because the angular frequency $\omega$ has the opposite sign in this dissertation. The Rayleigh integral (equation 2.13) simplifies to

$$
\tilde{p}=\overline{\rho_{0} c_{0} \frac{k}{k_{3}} e^{\mathrm{i} k_{3} x_{3}}} \cdot \tilde{v}_{n}
$$

where $\tilde{p}\left(k_{1}, k_{2}\right)$ and $\tilde{v}\left(k_{1}, k_{2}\right)$ are the 2D Fourier transforms of the field pressure and source velocity respectively.

The behavior of the waves in this straightforward equation can help to understand the behavior of complicated sound fields. The wave numbers $k_{1}$ and $k_{2}$ can take any real value because they are free parameters in the Fourier transform (equation 2.14). The square root in equation 2.16 can therefore be either real or imaginary. If $\left\{k_{1}, k_{2}\right\}$ lies in the radiation circle defined by $k_{1}^{2}+k_{2}^{2}<k^{2}$, then $k_{3}$ is real, leading to so-called propagating waves which do not decay. If $\left\{k_{1}, k_{2}\right\}$ lies outside the radiation circle then $k_{3}$ lies on the positive imaginary axis such that the pressure decays as a real exponential. These evanescent waves are depicted in figure 2.3(b).

The Fourier model is an exact representation of the Helmholtz equation in the wave number domain. Due to its simplicity, many important ideas were discovered first in the Fourier model. However, it should be noted that a source of finite size cannot generate purely propagating or evanescent waves. Acoustic waves always decay with the distance to the source.

The next section introduces numerical techniques to solve acoustic radiation problems without the Fourier transform. Fourier-based methods to solve forward and inverse acoustic problems are discussed in section 4.2. 


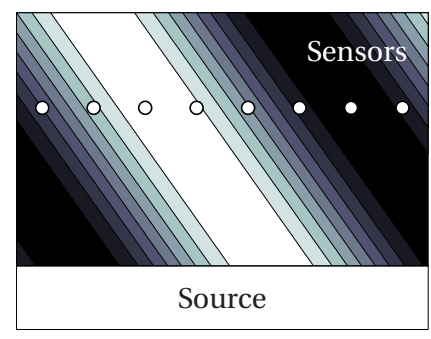

(a) A propagating wave

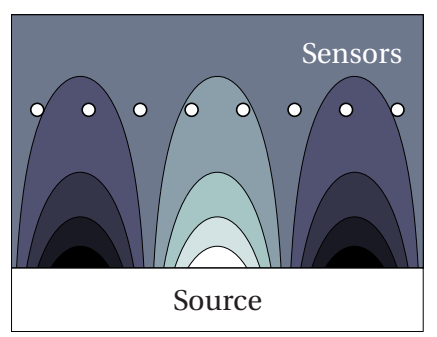

(b) An evanescent wave

Figure 2.3: The pressure field caused by a planar source in 3D (real part, cross section along a vertical plane). White (+) and Black (-) are scaled to the extremal values of the pressure field.

\subsection{Numerical acoustic modeling}

\subsubsection{Introduction}

Contrary to Fourier Acoustics, the boundary element method (BEM) is suitable to calculate sound fields if the normal velocity is prescribed at an arbitrary surface. This section introduces the discretization used in BEM. To explain the method in a straightforward way, the spatial discretization of the Rayleigh integral is considered here and not the discretization of the Helmholtz integral equation (HIE). The discretization can be extended to the HIE with minor modifications [89].

Discretization is the process of approximating a continuum problem by a discrete and finite set of equations. This can be achieved by introducing a mesh of quadrilateral or triangular elements with nodes at their corners. The normal velocity is then approximated in terms of shape functions (see figure 2.4). In BEM, the source geometry is also represented using a mesh. If the same shape functions are used for the geometry and the velocities, then the elements are said to be isoparametric.

\subsubsection{Discretization}

The Rayleigh integral can be discretized as follows. The normal velocity $v_{n}(\vec{y})$ at the source is approximated using a basis of shape functions $N_{j}(\vec{y})$ where $j \in\{1,2, \cdots, n\}$ (see also figure 2.4).

$$
v_{n}(\vec{y})=\sum_{j=1}^{n} N_{j}(\vec{y}) v_{j}
$$

Here $N_{j}(\vec{y})$ and $v_{j}$ are the shape function of degree of freedom $j$, and its contri- 


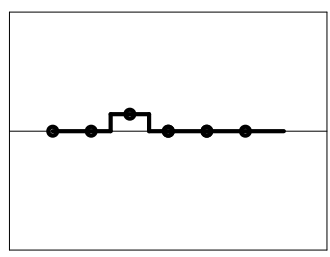

(a) Constant

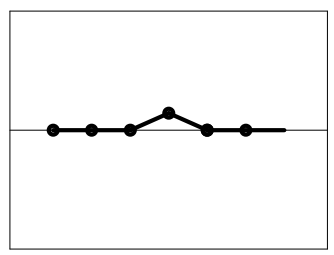

(b) Linear

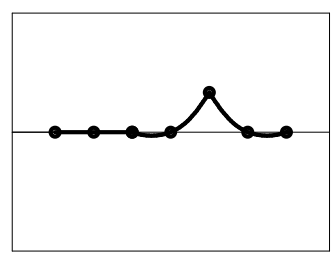

(c) Quadratic

Figure 2.4: Three examples of piecewise polynomial shape functions

bution to the normal velocity function $v_{n}(\vec{y})$. For the shape functions which are commonly used in practice, $v_{j}$ is the normal velocity at node $j$. The surface velocity can usually not be described exactly by the shape functions, which means 2.18 is usually an approximation. The Rayleigh integral (equation 2.12) is discretized as follows.

$$
\begin{aligned}
p(\vec{x}) & =\sum_{j} h_{j}(\vec{x}) v_{j} \quad \text { where } \\
h_{j}(\vec{x}) & =\frac{\mathrm{i} \omega \rho_{0}}{2 \pi} \int_{S} N_{j}(\vec{y}) \frac{e^{-\mathrm{i} k|\vec{y}-\vec{x}|}}{|\vec{y}-\vec{x}|} d S
\end{aligned}
$$

The integral in equation 2.20 can be evaluated numerically such that $p(\vec{x})$ can be calculated for any point $\vec{x}$ if the source velocity is known. However, the number of equations is still infinite if the pressure must be calculated over an entire area or volume. This part of the equation can be discretized by solving the equations at a finite set of points $\vec{x}_{i}, i \in\{1,2, \cdots, m\}$.

$$
p\left(\vec{x}_{i}\right)=\sum_{j} h_{j}\left(\vec{x}_{i}\right) v_{j}
$$

This is known as collocation. The location of the collocation points is best chosen in such a way that the gaps in the grid are as small as possible. In BEM, it is necessary to calculate the pressure at the source surface $S$. The collocation points $\vec{x}_{i}$ are often chosen as the source nodes in this case, because it leads to well-conditioned square matrices. The reader is referred to [12] for more details on collocation and other methods. In the rest of this dissertation, equation 2.21 will be written in matrixvector notation as follows

$$
\mathbf{f}=\mathbf{H}_{\mathbf{f s}} \mathbf{s} \text { where } \begin{cases}\mathbf{f}_{i} & =p\left(\vec{x}_{i}\right) \\ \left(\mathbf{H}_{\mathbf{f s}}\right)_{i j} & =h_{j}\left(\vec{x}_{i}\right) \\ \mathbf{s}_{j} & =v_{j}\end{cases}
$$


where $\mathbf{H}_{\mathbf{f s}} \in \mathbb{C}^{m \times n}$ is the transfer matrix, also known as the transfer function matrix [62] or frequency response function. The vectors $\mathbf{s}$ and $\mathbf{f}$ are termed the source and field vector respectively. In the inverse acoustic models which are based on the Rayleigh integral, the points $\vec{x}_{i}$ are the sensor locations. Since there is only a finite number $m$ of sensors, equation 2.19 simplifies to equation 2.21, where the nonphysical collocation points become physical sensor locations.

The Rayleigh integral and many other acoustic equations can be discretized and solved using a computer using the approach to discretization which has been outlined in this section. The discretization error tends to zero as the number of shape functions increases for many sets of shape functions. Hence, practically useful sets of shape functions strike a balance between good convergence properties and a small amount of time and memory necessary to solve the problem using a computer. It is also important that the shape functions are far from linearly dependent. Element methods are popular in numerical acoustics because they have these favourable properties.

In appendix A, a finite set of shape functions is derived which theoretically yields a discretization error of zero at the field points. Although this property is interesting from an academical point of view, the piecewise polynomial shape functions are considered to be more useful because in practice, these methods lead to faster calculations.

\subsection{Summary}

This chapter has introduced the theory of sound and vibration. The Helmholtz integral equation has been presented to give the relation between the vibrations or pressures at a source and the sound field that the source generates. This equation serves as the basis for the boundary element method (BEM) which is used in the next chapters.

The Rayleigh integral can be solved by applying the 2D spatial Fourier transform. The application of this equation to inverse problems will be discussed and analyzed in section 4.2.

Finally, the foundations of discretization have been discussed and applied to the Rayleigh integral. The discrete equations will be used throughout this thesis and efficient inverse techniques based on an element discretization of the Rayleigh integral are presented in section 4.3. 
CHAPTER 2 


\section{Chapter 3}

\section{Regularization}

\subsection{Introduction}

An inverse acoustic method uses acoustic measurement data at many points of the sound field to localize the acoustic sources. These sources are represented by vibrations at a predefined surface. Since inverse acoustic methods do not require the presence of a structure, the methods can localize aeroacoustic, thermal and structural sources as well as combinations of these three types. This can make the methods a vital tool in sound and vibration studies.

To calculate the source vibrations, the acoustic pressure or velocity is measured at the field points. The measurement data is processed and the inverse calculation is performed. This step consists of first calculating a forward model - which is the transfer matrix from the noise source to the sound field - and then inverting this matrix, such that the source vibrations are obtained by multiplying the processed

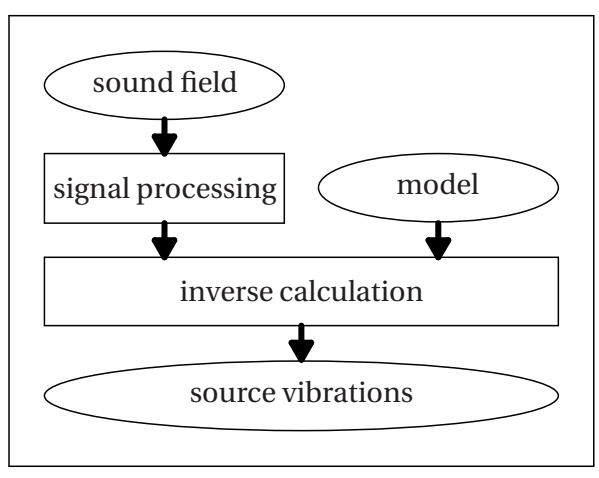

Figure 3.1: The steps required in inverse acoustics 
measurement data with the inverse transfer matrix. The result of the inverse calculation is the amplitude and phase at all points of the source surface. This general scheme has been depicted in figure 1.2. It is repeated in figure 3.1.

The forward model is the following matrix-vector equation in the frequency domain (see also equation 2.22)

$$
\mathbf{H}_{\mathbf{f s}} \mathbf{s}=\mathbf{f}
$$

where $\mathbf{H}_{\mathbf{f s}}(\omega) \in \mathbb{C}^{m \times n}$ is the transfer matrix and $\mathbf{f}(\omega) \in \mathbb{C}^{m}$ is the field vector containing acoustic measurement data. This vector can contain the pressure, the velocity in any direction or combinations of these quantities. The source vector $\mathbf{s}(\omega) \in \mathbb{C}^{n}$ contains the velocity or pressure for each degree of freedom at the source. Theoretically, it can also contain combinations of source quantities but this is unusual in practice.

The direct solution of equation 3.1 for the source vector $\mathbf{s}$ leads to unphysical results because the transfer matrix is badly ill-conditioned. Moreover, the conditioning of the matrix worsens as the number of source and field points increases. In the limit case where the source and field vectors become functions on a continuous surface the problem is even ill-posed. Hence the inverse acoustic problem is a discretized ill-posed problem, also referred to as a discrete ill-posed problem [28].

Although discrete ill-posed problems cannot be solved exactly, a practically useful approximation can be calculated. Methods that calculate these solutions are called regularization methods. The singular value decomposition (SVD) is a useful mathematical tool to analyze inverse problems and to develop regularization methods.

This chapter introduces SVD-based techniques to study and solve inverse problems. Section 3.2 introduces the basics of SVD-based regularization and section 3.3 generalizes the SVD to alleviate some of its limitations. The theoretical framework is applied to an example in section 3.4 and fast numerical algorithms for regularization problems are discussed in section 3.5.

\subsection{The SVD applied to regularization problems}

The SVD gives insight into the ill-conditioned nature of discrete ill-posed problems because it makes every matrix diagonal by using the proper orthogonal bases for the source and field vectors [84]. The SVD of the transfer matrix $\mathbf{H}_{\mathbf{f s}} \in \mathbb{C}^{m \times n}$ is given the following expression.

$$
\mathbf{H}_{\mathbf{f s}}=\mathbf{U} \boldsymbol{\Sigma} \mathbf{V}^{H} \text { with }\left\{\begin{array}{l}
\mathbf{U}^{H} \mathbf{U}=\mathbf{I} \\
\mathbf{V}^{H} \mathbf{V}=\mathbf{I}
\end{array}\right.
$$


The number of singular values is $k=\min (m, n)$. Furthermore, $\mathbf{U} \in \mathbb{C}^{m \times k}$ and $\mathbf{V} \in$ $\mathbb{C}^{n \times k}$ are the matrices with the left and right singular vectors respectively. $\Sigma \in \mathbb{R}^{k \times k}$ is a diagonal matrix containing singular values and $\mathbf{I} \in \mathbb{R}^{k \times k}$ is the identity matrix of size $k$. A useful consequence of equation 3.2 is the fact that the eigenvalue decompositions of the Hermitian matrices $\mathbf{H}_{\mathbf{f s}} \mathbf{H}_{\mathbf{f s}}^{H}$ and $\mathbf{H}_{\mathbf{f s}}^{H} \mathbf{H}_{\mathbf{f s}}$ are

$$
\begin{aligned}
& \mathbf{H}_{\mathbf{f s}} \mathbf{H}_{\mathbf{f s}}^{H}=\mathbf{U} \boldsymbol{\Sigma} \boldsymbol{\Sigma}^{H} \mathbf{U}^{H} \\
& \mathbf{H}_{\mathbf{f s}}^{H} \mathbf{H}_{\mathbf{f s}}=\mathbf{V} \boldsymbol{\Sigma}^{H} \boldsymbol{\Sigma} \mathbf{V}^{H}
\end{aligned}
$$

where $\mathbf{U}$ and $\mathbf{V}$ are the matrices containing eigenvectors and $\Sigma \Sigma^{H}=\Sigma^{H} \boldsymbol{\Sigma} \in \mathbb{C}^{k \times k}$ is a diagonal matrix containing the eigenvalues.

Equation 3.2 makes it possible to rewrite equation 3.1 as

$$
\begin{aligned}
\mathbf{U} \Sigma \mathbf{V}^{H} \mathbf{s} & =\mathbf{f} \\
\Sigma \mathbf{s}^{\prime} & =\mathbf{f}^{\prime} \text { with }\left\{\begin{array}{l}
\mathbf{U} \mathbf{f}^{\prime}=\mathbf{f} \\
\mathbf{V \mathbf { s } ^ { \prime }}=\mathbf{s}
\end{array}\right. \\
\sigma_{i} \mathbf{s}_{i}^{\prime} & =\mathbf{f}_{i}^{\prime}
\end{aligned}
$$

Equation 3.6 shows the change of basis that makes the transfer matrix diagonal and equation 3.7 can be solved for $\mathbf{s}_{i}^{\prime}$ one variable at a time. In practice, a vector of noisy measurement data $\widehat{\mathbf{f}}$ is known instead of the exact vector $\mathbf{f}$. In inverse acoustics, the singular values $\sigma_{i}$ decay to zero with increasing $i$. Hence, any noise in $\widehat{\mathbf{f}}_{i}^{\prime}$ is amplified many times in the approximate solution $\widehat{\mathbf{s}}_{i}^{\prime}$. Although there is no way to find an accurate estimate for this value $\widehat{\mathbf{s}}_{i}^{\prime}$ based on the measurement data, these components may be filtered out such that the solution is not overshadowed by noise. This is achieved by applying a regularization filter to the equation

$$
\widehat{\mathbf{s}}_{i}^{\prime \alpha}=\frac{\mathscr{F}_{\alpha}\left(\sigma_{i}\right)}{\sigma_{i}} \widehat{\mathbf{f}}_{i}^{\prime}
$$

where $\alpha$ is the regularization parameter, which depends upon the amount of noise in the measurement data. The filter function $\mathscr{F}_{\alpha}\left(\sigma_{i}\right)$ is chosen such that it is close to one for large $\sigma_{i}$ and smaller than $\sigma_{i}$ for small $\sigma_{i}$. Two filters which are commonly used in practice are

$$
\begin{array}{ll}
\text { TSVD } & \mathscr{F}_{\alpha}\left(\sigma_{i}\right)=\left\{\begin{array}{lll}
1 & \text { if } & \sigma_{i} \leq \alpha \\
0 & \text { if } & \sigma_{i}>\alpha
\end{array}\right. \\
\text { Tikhonov } & \mathscr{F}_{\alpha}\left(\sigma_{i}\right)=\frac{\sigma_{i}^{2}}{\sigma_{i}^{2}+\alpha^{2}}
\end{array}
$$

TSVD stands for Truncated Singular Value Decomposition. It is also known as singular value discarding. Both methods have been studied intensively by mathematicians and engineers. An extensive survey of the mathematical properties of the various filter functions is given in [21]. 
The inverse solution can be found by multiplying the source vector $\widehat{\mathbf{s}}^{\prime \alpha}$ by the matrix of right singular vectors $\mathbf{V}$. The entire operation of transforming the matrix to the basis of singular vectors, calculating the regularized solution and transforming back to the spatial domain can be expressed as a multiplication by the regularized inverse matrix.

$$
\begin{aligned}
\widehat{\mathbf{s}}^{\alpha} & =\mathbf{H}_{\mathbf{f s}}^{\dagger \alpha} \widehat{\mathbf{f}} \quad \text { with } \\
\mathbf{H}_{\mathbf{f s}}^{\dagger \alpha} & =\mathbf{V} \boldsymbol{\Sigma}^{\dagger \alpha} \mathbf{U}^{H}
\end{aligned}
$$

Where $\Sigma^{\dagger \alpha} \in \mathbb{C}^{k \times k}$ is a diagonal matrix with diagonal elements $\mathscr{F}_{\alpha}\left(\sigma_{i}\right) / \sigma_{i}$.

\subsection{Alternative SVDs}

The pursuit of better results has been an important driving force in the field of inverse problems. There are some applications where filters applied to the conventional SVD do not provide the best known results. This section describes the limitations of the SVD and generalizes the SVD to deal with some of these limitations.

To derive alternative SVDs, consider the following expression for the first singular value, which is equal to the first singular value in equation 3.2 [39].

$$
\sigma_{1}=\sup \frac{\left\|\mathbf{H}_{\mathbf{f s}} \mathbf{s}\right\|_{2}}{\|\mathbf{s}\|_{2}} \quad \text { where }\left\{\begin{array}{l}
\|\mathbf{s}\|_{2}^{2}=\sum_{j=1}^{n}\left|s_{j}\right|^{2} \\
\|\mathbf{f}\|_{2}^{2}=\sum_{i=1}^{m}\left|f_{i}\right|^{2}
\end{array}\right.
$$

Where sup denotes the supremum, which is equal to the maximum if the maximum exists. The vector $\mathbf{s}$ which achieves this maximum is the first right singular vector. As can be seen in equation 3.12, it radiates sound most efficiently to the field points, where the efficiency is measured as the ratio of the 2-norm $\mathbf{f}$ compared to the 2norm of $\mathbf{s}$. The second singular vector radiates sound most effectively, but it is orthogonal to the first. The third is the most efficient radiator orthogonal to the first two, and so on. Applying a filter to the SVD is therefore the same as filtering out those components of the source vibrations that radiate sound so inefficiently that the measurement data are likely to be dominated by noise.

The limitation of the SVD is that the radiation efficiency is measured using the 2 -norms of $\mathbf{s}$ and $\mathbf{f}$. If these norms are unphysical then the regularization results are unphysical as well. More meaningful results can be achieved by changing the norm of the source vector or the norm of the field vector. There is no universally accepted way to derive a suitable norm for a specific problem. Instead, it is necessary to compare simulation results of various norms and to determine which norms lead to the most accurate and useful results. Clearly, there are infinitely many norms and only a few can be compared. This chapter uses some straightforward norms 


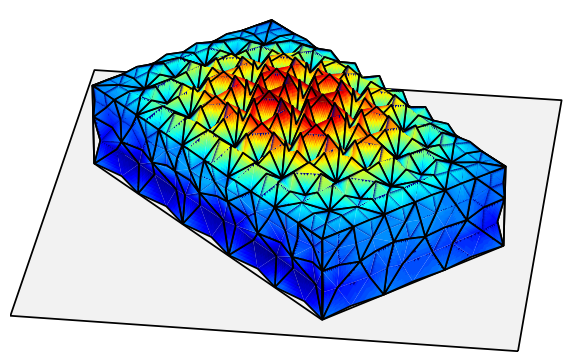

(a) Singular vector without weighting

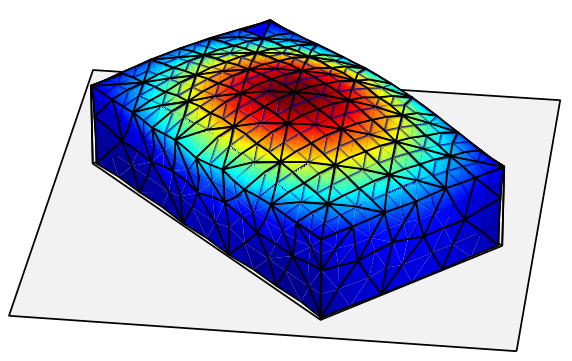

(b) Singular vector with weighting

Figure 3.2: Real part of the first (right) singular vector based on quadratic elements. Images from Visser [89].

from functional analysis, but statistical arguments can also be used to determine which norms are suitable [80].

The generalized singular value decomposition (GSVD) can be used to change one of these norms but a further generalization of the SVD is necessary to change both norms.

A practical example of a regularization problem where the 2-norm is not applicable is IBEM using quadratic elements (see figure 3.2). In that case, a unit displacement of some specific degrees of freedom represents a larger displacement than on other degrees of freedom, such that the basic SVD yields a first right singular vector where the degrees of freedom that bring about small displacements are suppressed. This causes unphysical inverse results. Visser suggests that a more applicable norm is the mean squared normal velocity of the surface [91, 90]. This norm is used in section 3.4. In some inverse problems, the relevant norm involves a discrete derivative or differential equation. Section 3.4 describes the case where the source norm involves a derivative such that the SVD is based on how efficiently pressure is radiated to the field nodes compared to the smoothness of the source vibrations.

Inaccurate results can also be caused by the fact that the field norm $\|\mathbf{f}\|_{2}$ is not an applicable measure of the measurement noise of the field data. For example, if both pressure and velocity have been measured then a noise level of $10^{-5} \mathrm{~Pa}$ and $10^{-5} \mathrm{~m} / \mathrm{s}$ are not necessarily the same. There may also be some correlation between the measurement noise at different points.

An applicable SVD can be defined in all of these cases. The definitions are as follows

$$
\sigma_{1}=\sup \frac{\left\|\mathbf{H}_{\mathbf{f s}} \mathbf{s}\right\|_{\mathbf{f}}}{\|\mathbf{s}\|_{\mathbf{s}}} \text { where }\left\{\begin{aligned}
\|\mathbf{s}\|_{\mathbf{s}}^{2} & =\mathbf{s}^{H} \mathbf{W}_{\mathbf{s s}} \mathbf{s} \\
\|\mathbf{f}\|_{\mathbf{f}}^{2} & =\mathbf{f}^{H} \mathbf{W}_{\mathbf{f f}} \mathbf{f}
\end{aligned}\right.
$$


where it is necessary to choose the weighting matrices $\mathbf{W}_{\mathbf{s s}}$ and $\mathbf{W}_{\mathbf{f f}}$. Although it is difficult to determine which is the best weighting matrix, they must be square, Hermitian and positive definite. Examples of specific weighting matrices are given in section 3.4.

A straightforward way to solve the SVD of equation 3.13 is to transform it to standard form. The transformation based on two weighting matrices is not wellknown in the literature but it it is implied by Hansen [28] (sections 2.3 and 5.1.1). A linear transform is applied to the source and field vectors such that the SVD in equation 3.13 can be solved using 2-norms. The transformed source and field vectors $\mathbf{s}_{*}$ and $\mathbf{f}_{*}$ are written as:

$$
\begin{array}{lllllll}
\mathbf{s}_{*} & =\mathbf{B}_{\mathbf{s}} \mathbf{s} & \text { such that } & \left\|\mathbf{s}_{*}\right\|_{2} & =\|\mathbf{s}\|_{\mathbf{s}} & \forall & \mathbf{s} \\
\mathbf{f}_{*} & =\mathbf{B}_{\mathbf{f}} \mathbf{f} & \text { such that } & \left\|\mathbf{f}_{*}\right\|_{2} & =\|\mathbf{f}\|_{\mathbf{f}} & \forall & \mathbf{f}
\end{array}
$$

Combining equations 3.12 through 3.14, the following equations must hold for the transform matrices $\mathbf{B}_{\mathbf{s}}$ and $\mathbf{B}_{\mathbf{f}}$

$$
\mathbf{W}_{\mathbf{s s}}=\mathbf{B}_{\mathbf{s}}^{H} \mathbf{B}_{\mathbf{s}} \quad \mathbf{W}_{\mathbf{f f}}=\mathbf{B}_{\mathbf{f}}^{H} \mathbf{B}_{\mathbf{f}}
$$

The matrices $\mathbf{B}_{\mathbf{f}}$ and $\mathbf{B}_{\mathbf{s}}$ are not defined uniquely by equation 3.15 but all decompositions of this form lead to the same regularized solution. Examples of such decompositions are the Cholesky decomposition and the matrix exponential $\mathbf{B}_{\mathbf{s s}}^{1 / 2}$ (see e.g. [57]).

Next, the transfer matrix between the two transformed vectors is calculated. The forward model (equation 3.1) becomes

$$
\begin{aligned}
\mathbf{H}_{*} \mathbf{s}_{*} & =\mathbf{f}_{*} \quad \text { where } \\
\mathbf{H}_{*} & =\mathbf{B}_{\mathbf{f}} \mathbf{H}_{\mathbf{f s}} \mathbf{B}_{\mathbf{s}}^{-1}
\end{aligned}
$$

Using equation 3.2, the ordinary SVD of the transformed matrix $\mathbf{H}_{*}$ is

$$
\mathbf{H}_{*}=\mathbf{U}_{*} \boldsymbol{\Sigma}_{*} \mathbf{V}_{*}^{H} \text { with } \begin{cases}\mathbf{U}_{*}^{H} \mathbf{U}_{*}=\mathbf{I} \\ \mathbf{V}_{*}^{H} \mathbf{V}_{*}=\mathbf{I}\end{cases}
$$

The inverse solution can be calculated using the techniques of section 3.2.

This concludes a summary of the theoretical basis of regularization. The next section compares two different source norms. In the other IBEM results in this thesis, the source norm is the mean-squared normal velocity norm and the field norm is the 2-norm. Although various other norms have been implemented and tested for each of the topics in this study, these norms have proven to give the most reliable results. 


\subsection{Examples}

\subsubsection{Problem description}

The next example will be used throughout this thesis. The source represents a hard disk drive. Experimental data and inverse solutions of this source are considered in chapter 4 .

The source is a rectangular box of $147 \times 102 \times 26 \mathrm{~mm}$ (see figure 3.3). Pressure is measured at a distance of $40 \mathrm{~mm}$ from the source surface and the field grid spans a rectangular area of $160 \times 200 \mathrm{~mm}$ with a grid spacing of $10 \mathrm{~mm}$. The number of degrees of freedom in the boundary element model is 2106 and the number of field points is 357. The transfer matrices have been calculated using an in-house BEM code [90]. The used frequency is $5 \mathrm{kHz}$. The acoustic wavelength is $68 \mathrm{~mm}$ at this frequency, which is approximately half the length of the source.

\subsubsection{Norms and singular vectors}

To give examples of the singular vectors and the effect of regularization, the weighting matrices must be chosen. The field weighting matrix $\mathbf{W}_{\mathbf{f f}}$ is chosen to be the identity matrix. Two source norms are compared. The norm $A$ is the mean squared velocity norm, which is simply the $L_{2}(S)$ norm of the source velocity. It is defined to be:

$$
\|x\|_{A}^{2} \equiv \frac{1}{|S|} \int_{S}\left|v_{n}(\vec{y})\right|^{2} d S
$$

where $S$ is the source surface and $|S|$ is its area. In the discrete case, the source weighting matrix $\mathbf{W}_{\mathbf{s s}}$ can be expressed in terms of shape functions (see also equation 2.18). The source weighting matrix becomes

$$
\left(\mathbf{W}_{A}\right)_{i j}=\frac{1}{|S|} \int_{S} N_{i}(\vec{y}) N_{j}(\vec{y}) d S
$$

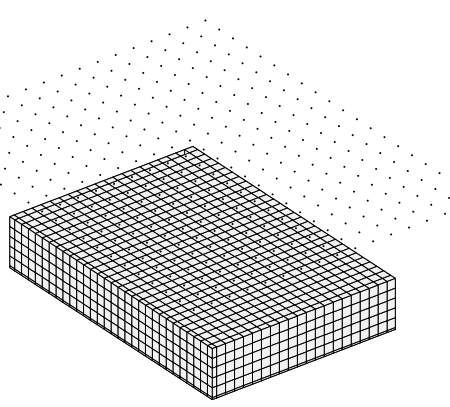

Figure 3.3: Geometry of the boundary element model with source elements and field points 
where $N_{i}(\vec{y})$ is the shape function of degree of freedom $i$. This norm will be used in the rest of this dissertation.

Norm B is based on the mean squared derivative of the normal velocity.

$$
\|x\|_{B}^{2} \equiv \frac{1}{|S|} \int_{S}\left|\frac{\partial v_{n}(\vec{y})}{\partial \vec{y}_{1}}\right|^{2}+\left|\frac{\partial v_{n}(\vec{y})}{\partial \vec{y}_{2}}\right|^{2} d S
$$

Where $\vec{y}_{1}$ and $\vec{y}_{2}$ are perpendicular coordinate axes along the surface. At the edges of the structure, the normal velocity is constrained to zero to make sure that a solution with a norm of zero also has a velocity vector of zero. The discrete form of 3.20 is as follows.

$$
\left(\mathbf{W}_{B}\right)_{i j}=\frac{1}{|S|} \int_{S} \frac{\partial N_{i}(\vec{y})}{\partial \vec{y}_{1}} \frac{\partial N_{j}(\vec{y})}{\partial \vec{y}_{1}}+\frac{\partial N_{i}(\vec{y})}{\partial \vec{y}_{2}} \frac{\partial N_{j}(\vec{y})}{\partial \vec{y}_{2}} d S
$$

Gaussian integration is applied to evaluate the integrals on a computer. The numerical procedures are considered well-known because the same integrals follow from the Finite Element Method applied to the 2D Laplace problem [12].

The singular vectors for the two norms are depicted in figures 3.4 and 3.5. Although the singular vectors have an arbitrary complex angle, they are nearly real except for a single complex constant for all nodes. Each of the singular vectors has therefore been multiplied by a constant that maximizes the real part and that real part has been plotted. The singular vectors in figure 3.4 radiate sound most efficiently to the sensors in terms of the mean squared velocity. The singular vectors in figure 3.5 radiate sound most efficiently in terms of the mean squared derivative of the velocity. Since the efficiency is measured based on the derivative, the normal velocity has a much smoother shape.

\subsubsection{The inverse acoustic calculation}

To illustrate the inverse acoustic calculation, a simulation is performed using a point source at the center of the front surface of the hard disk drive. Figure 3.6(a) shows the transformed field data ( $f_{i}^{\prime}$ in equation 3.7) for the mean squared velocity norm. In the absence of noise, the values decay steadily for higher singular values but they reach a noise floor if $1 \%$ of noise is added. The source solution is found by dividing the field data by the singular values depicted in figure 3.6(b). Although the contributions of the first singular vectors are accurate, the contributions of the later singular vectors rise steadily because they are the result of a constant noise floor divided by the decreasing singular values. A good compromise is made by using the first 60 out of the total 357 singular values. The other contributions are dominated by noise. 


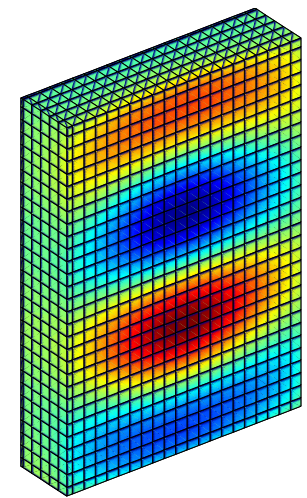

(a) first

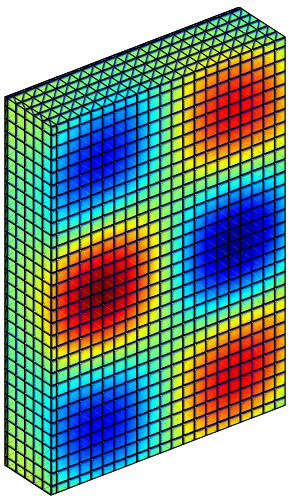

(b) second

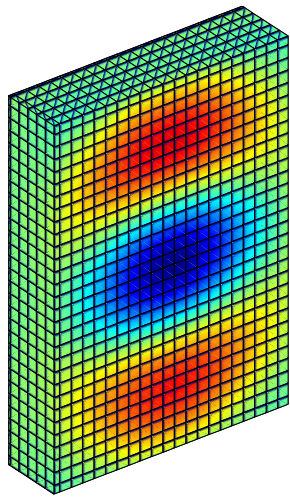

(c) third

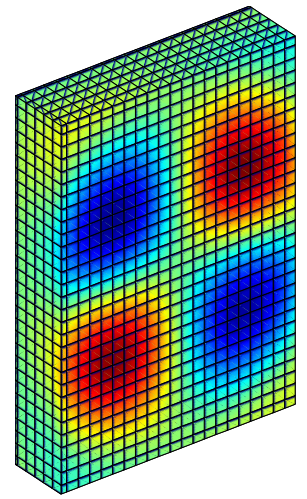

(d) fourth

Figure 3.4: First four (right) singular vectors using norm A in accordance to equation 3.18 (Normal velocity, $5 \mathrm{kHz}$ ).

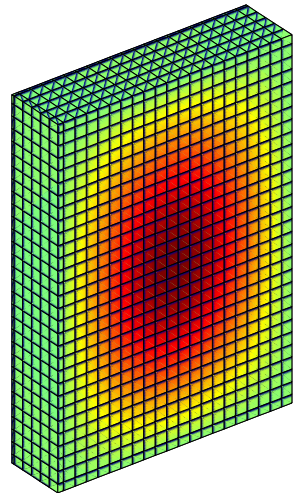

(a) first

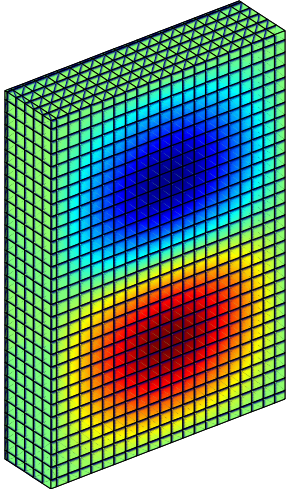

(b) second

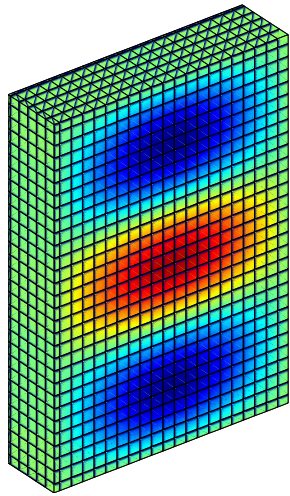

(c) third

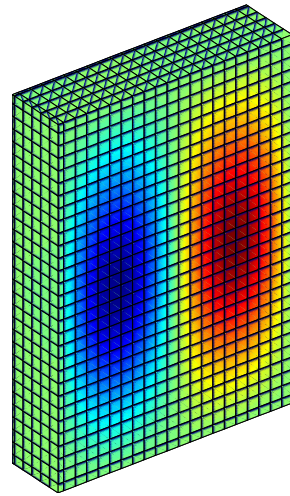

(d) fourth

Figure 3.5: First four (right) singular vectors using norm B in accordance to equation 3.20. (Normal velocity, 5kHz) 

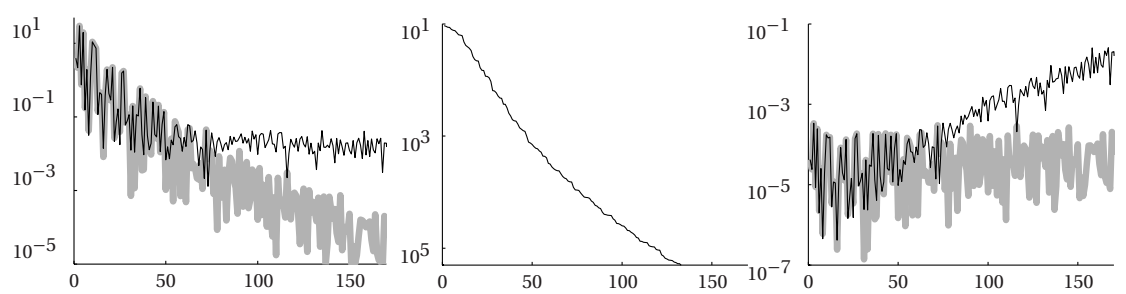
(a) field data. Exact
(b) Singular values
(c) Source data. Exact $(-)$ and with noise
$(-$ )and with noise

Figure 3.6: The inverse acoustic process in terms of singular values. (Norm A in accordance to equation 3.18)
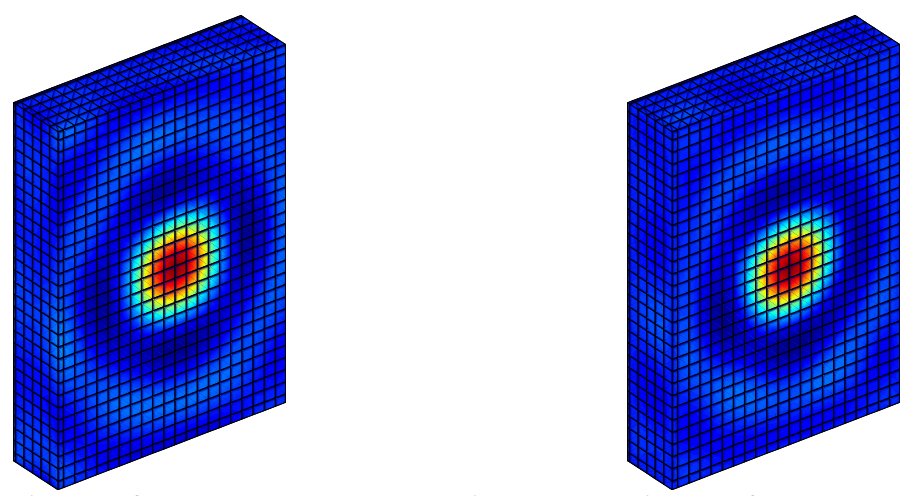

(a) Inverse solution of a point source (TSVD). (b) Inverse solution of a point source (TSVD). Norm A, in accordance to equation 3.18 (Nor- Norm B, in accordance to equation 3.20 (Normal velocity, $5 \mathrm{kHz}$ ) mal velocity, $5 \mathrm{kHz}$ )

Figure 3.7: Inverse solution of a point source using different norms 


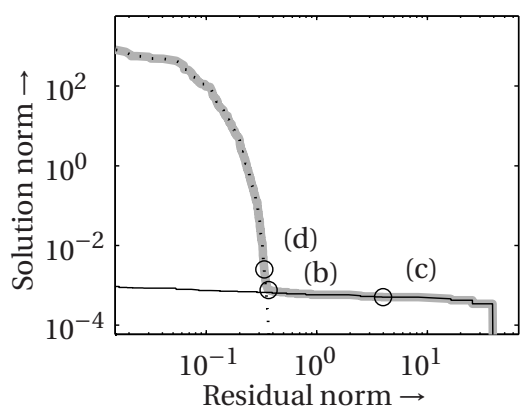

Figure 3.8: L-curve of measurement data with noise ( $\square)$, without noise ( - ) and only noise $(\cdots)$.

The result is depicted in figure 3.7(a). The same simulation is also performed using norm B (equation 3.20). It is depicted in figure 3.7(b). The results are similar, which is surprising at first glance because the singular vectors of the two norms do not look alike (compare figures 3.4 and 3.5). Nevertheless, regularization removes the highest singular vectors in both cases. Since the first singular vectors are smooth and the last singular vectors are oscillatory for both norms, the subspace spanned by the singular vectors, and hence the calculated result, is similar. The oscillatory components are removed, leading to a smoothed, or spatially bandlimited, version of the original point source.

\subsubsection{The L-curve}

The L-curve is a graphical aid to select the regularization parameter which was introduced by Hansen in $1992[27,30]$. It is a plot of the solution norm $\left\|\widehat{\mathbf{s}}^{\alpha}\right\|_{\mathbf{s}}$ versus the residual norm $\left\|\mathbf{H}_{\mathbf{f s}} \widehat{\mathbf{s}}^{\alpha}-\widehat{\mathbf{f}}\right\|_{\mathbf{f}}$. When plotted on a log-log scale, the graph tends to have a characteristic L-shaped appearance (see figure 3.8). On the horizontal part, the solutions tend to be over-smoothed: too many components are filtered out such that the filter itself causes inaccuracies. This part of the curve is close to the L-curve of the measurement data without noise because the components that are not removed have a good signal to noise ratio. The vertical part solutions are under-smoothed, not enough components are filtered out such that the solution is dominated by noise. This part of the curve is close to the L-curve of the noise. A good compromise between undersmoothing and oversmoothing can be found in the corner of the graph (see figure 3.9).

The trends of the L-curve can be seen in almost any inverse problem and they have been studied extensively in the field of inverse problems, but they are no more than trends [21]. The best solution can theoretically lie far away from the corner 


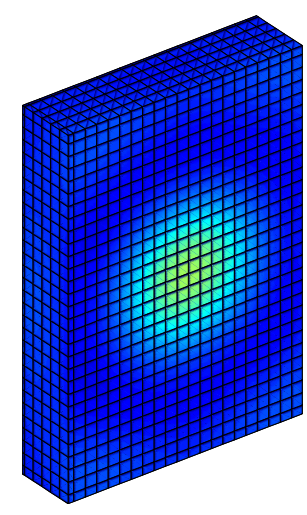

(a) 13 vectors

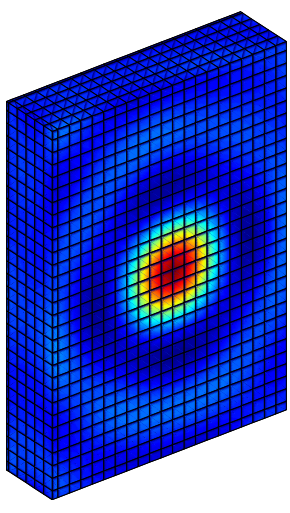

(b) 60 vectors

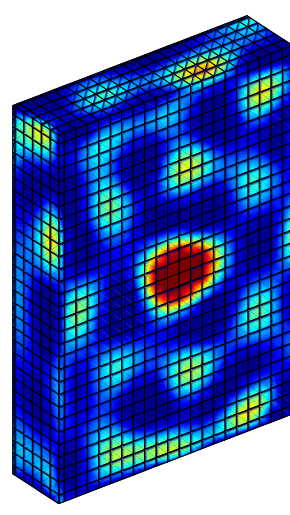

(c) 100 vectors

Figure 3.9: Three inverse solutions (real part). Figure 3.9(b) is the same as figure 3.7(a). The color scaling used in the plots is the same 3.7(a).

of the L-curve and a regularization method that produces the lowest L-curve does not necessarily provide the most accurate results. Many authors advocate methods that select the corner automatically based on a more detailed analysis of the trends (see [30, 21] for surveys of these methods). These automatic methods have been studied extensively, both from a theoretical and practical point of view. Here, the L-curve is used as a graphical aid only. The regularization parameter is selected using a point-and-click interface, such that the user has the opportunity to judge the quality of several solutions.

This concludes the examples of the inverse acoustic calculations. The next section introduces a class of fast algorithms for inverse acoustic calculations.

\subsection{Numerical implementation}

\subsubsection{Introduction}

Software packages to calculate the SVD are widely available. It is supported by the numerical library LAPACK [1] which can be accessed from many programming languages including MATLAB. The numerical implementation of regularization software is therefore straightforward once the transfer matrix and the applicable matrices for the source and field norm are available.

The class of Krylov subspace iteration methods is considered in this section. This class consists of methods for least squares problems as well as methods to determine various factorizations such as the SVD. Contrary to most SVD algorithms, Krylov subspace SVD algorithms approximate a few of the first singular values and 
-vectors, whereas other SVD algorithms estimate all of the singular values and vectors. Only the largest singular vectors are necessary for inverse acoustics such that these methods are more efficient.

A second attractive property of Krylov subspace methods is the fact that they use matrix-vector multiplications to determine the solution instead of the matrix coefficients themselves. Since the many matrix-vector multiplications dominate the amount of computational effort required for these methods, they are extremely efficient if a fast algorithm is available for the matrix-vector multiplications. One such algorithm is discussed in section 4.3.3. A comparison of the computation times of various regularization algorithms is given in section 4.4.2.

This section considers the application of Krylov subspace methods to IBEM. Methods for least squares problems are discussed in section 3.5.2 and methods to calculate the SVD are discussed in section 3.5.3.

\subsubsection{Least squares and Krylov subspace}

The class of Krylov subspace iteration methods is widely used to solve least squares problems and other linear problems. Two examples of these algorithms are the conjugate gradients least squares algorithm (CGLS) and the more stable least squares QR (LSQR) algorithm [4].

As the name suggests, Krylov subspace iteration methods construct a basis for Krylov subspace. In the first iteration, this subspace is one dimensional and in each of the following iterations, a dimension is added to this subspace and the problem is projected onto a new, larger subspace. At each iteration $k$, the iterands $\mathbf{s}_{k}$ are in accordance to the Galerkin condition, which states that the residuals $\mathbf{r}_{k}$ are orthogonal to Krylov subspace [75].

$$
\mathbf{r}_{k} \perp \mathscr{K}_{k}\left(\mathbf{H}_{\mathbf{s f}}^{H} \mathbf{H}_{\mathbf{f s}}, \mathbf{H}_{\mathbf{f s}}^{H} \widehat{\mathbf{f}}\right) \quad \text { with } \quad \mathbf{r}_{k}=\mathbf{H}_{\mathbf{s f}}^{H} \mathbf{H}_{\mathbf{f s}} \mathbf{s}_{k}-\mathbf{H}_{\mathbf{f s}} \widehat{\mathbf{f}}
$$

where $\mathscr{K}_{k}\left(\mathbf{H}_{\mathbf{s f}}^{H} \mathbf{H}_{\mathbf{f s}}, \mathbf{H}_{\mathbf{f s}}^{H} \widehat{\mathbf{f}}\right)$ is Krylov subspace at iteration $k$. It is defined to be [28]

$$
\begin{aligned}
& \mathscr{K}_{k}\left(\mathbf{H}_{\mathbf{s f}}^{H} \mathbf{H}_{\mathbf{f s}}, \mathbf{H}_{\mathbf{f s}}^{H} \widehat{\mathbf{f}}\right) \equiv \\
& \quad \operatorname{span}\left\{\mathbf{H}_{\mathbf{f s}}^{H} \widehat{\mathbf{f}},\left(\mathbf{H}_{\mathbf{f s}}^{H} \mathbf{H}_{\mathbf{f s}}\right) \mathbf{H}_{\mathbf{f s}}^{H} \widehat{\mathbf{f}}, \ldots,\left(\mathbf{H}_{\mathbf{f s}}^{H} \mathbf{H}_{\mathbf{f s}}\right)^{k-1} \mathbf{H}_{\mathbf{f s}}^{H} \widehat{\mathbf{f}}\right\}
\end{aligned}
$$

This problem can be solved efficiently using stright-forward algorithms. In the current context, the most important aspect of the Krylov subspace solvers is the fact that they have an inherent regularizing property. Each iterand is a regularized solution and as the iterations continue, the smoothing decreases. Theoretically, the iterands converge to the unsmoothed least squares solution as the process continues but in practice the finite precision of computer hardware can cause the solution 


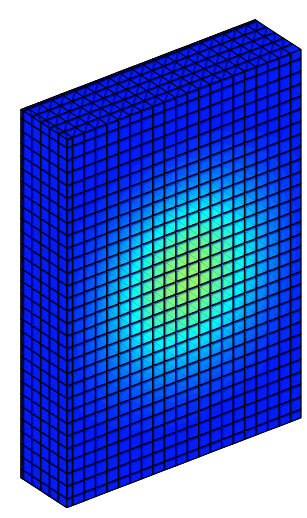

(a) one iteration

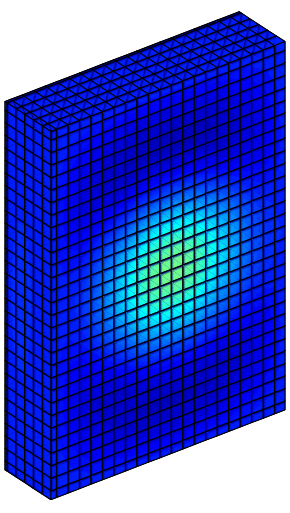

(b) two iterations

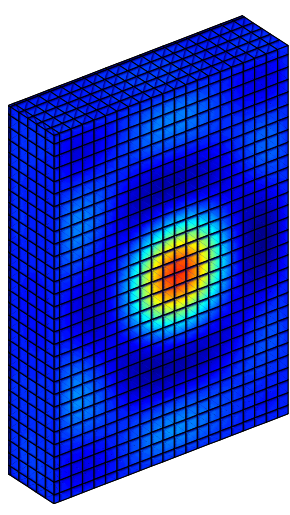

(c) 20 iterations

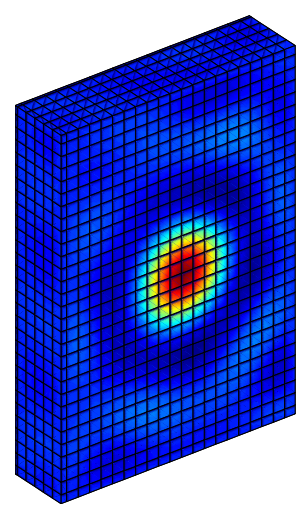

(d) 70 iterations, TSVD using 60 vectors

Figure 3.10: Inverse solutions from a Krylov subspace solver (LSQR). (normal velocity, $5 \mathrm{kHz}$, Colors are scaled as in figure 3.7). The derivative norm (equation 3.20) has been used.

process to stagnate. In other fields of research, this problem is often solved by reenforcing the orthogonality relations between the iterands, but this is usually not necessary in inverse acoustics.

The results of all iterations are used to plot the L-curve or to select a suitable regularization parameter by any other means. Since all regularized results are calculated by one run of the algorithm, the methods are faster than most other methods. In practice, the results are as good as the results achieved by TSVD and they are often almost indistinguishable to the naked eye [90]. The reader is referred to [21] for a detailed mathematical study of regularizing property of the Krylov subspace methods.

In figure 3.10, the iterands of LSQR are depicted for the point source problem of figure 3.7. The results of iterations 1 and 2 are clearly oversmoothed and a good approximation is achieved at iteration 20. The source depicted in figure 3.10(d) is calculated using the Lanczos SVD algorithm which is discussed in the next section.

\subsubsection{The SVD and Krylov subspace}

The SVD is indispensible for some problems. This decomposition makes it possible to apply any filter to the singular values without any further theoretical developments. The SVD is also necessary to efficiently implement the regularization methods for cross spectra discussed in chapter 6. 


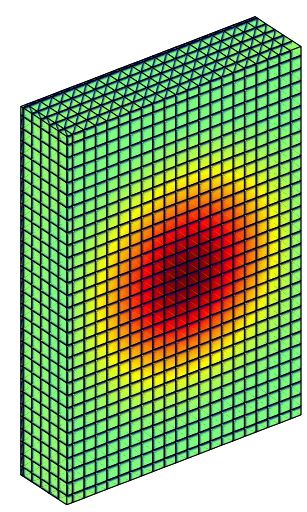

(a) one iteration

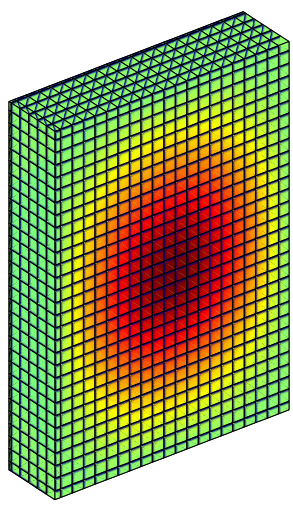

(b) two iterations

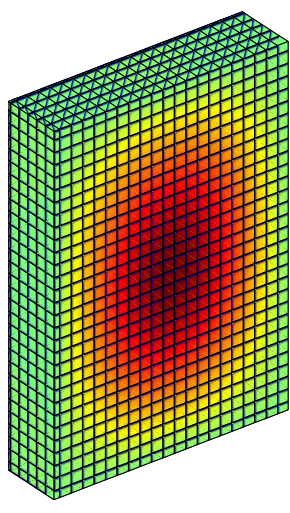

(c) three iterations

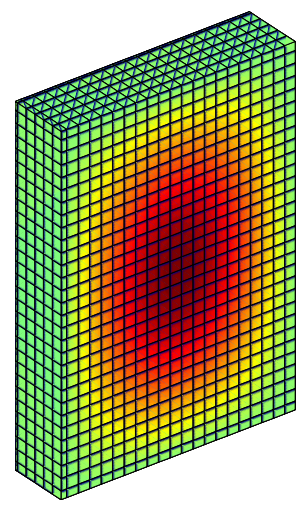

(d) exact solution

Figure 3.11: Approximations of the first singular vector using a Krylov subspace solver using the norm of equation 3.20 .

Since only the largest singular values, and the corresponding singular vectors are necessary for inverse acoustics, a Krylov solver is a computationally attractive alternative to other SVD methods. An applicable Krylov solver for this problem is the Lanczos SVD [26]. The method is by no means new in applied mathematics and it is even mentioned in the influential reference work by Hansen [28] but to the author's knowledge, it is new in inverse acoustics.

The Lanczos SVD consists of the Lanczos bidiagonalization algorithm followed by the SVD of the resulting bidiagonal matrix. The QR SVD algorithm can be used for this second problem. The number of singular vectors which is calculated is equal to the number of iterations $k$, but the smaller singular values and their corresponding vectors tend to be inaccurate. The number of accurate singular values and -vectors increases with the number of iterations. The singular values all become exact when the iteration number is equal to the rank of the matrix, but these solvers are truly competitive when the process is terminated earlier, yielding a good approximation rather than an exact solution.

To show the effectiveness of the Lanczos-SVD, the first three approximations of the largest singular vector according to norm B (the derivative norm) are depicted in figure 3.11. The first singular vector of norm A (the $L_{2}$ norm) requires more iterations to converge because it is skew-symmetric and the sound field is almost symmetric.

If the velocities at the source are not all correlated to each other, as discussed in section 5.3.3, then the right-hand side $\mathbf{f}$ can be represented as a matrix which contains a column for each of the uncorrelated sources. If only one of these columns is used, the SVD represents the inverse solution of the other columns inaccurately. 
If its number of columns is small compared to its number of rows, then the SVD can be solved efficiently using the block Lanczos SVD, which uses all columns of the right-hand side [20].

The Lanczos-SVD (s well as the block-Lanczos-SVD have been implemented and tested in MATLAB and many of the images in this thesis have been made using that software.

\subsection{Summary}

This chapter has introduced the singular value decomposition (SVD) as a way to analyze and solve inverse problems. Although the inverse acoustic problem cannot be solved exactly, a practically useful solution can be calculated using SVD-based regularization.

Although every matrix has an SVD, this decomposition does not necessarily lead to meaningful results. The regularization results can sometimes be improved by choosing a meaningful inner product and norm for the source and field vectors. A new transfer matrix with a meaningful SVD can then be derived.

The class of Krylov subspace methods is useful to calculate a regularized inverse solution. These methods have been used to solve systems of equations and least squares problems in acoustics. A new aspect of this dissertation is the application of the Lanczos SVD to the inverse acoustical problem. The algorithm is often faster than other regularization methods in general and it is extremely efficient if a fast algorithm is available for matrix-vector multiplication. One such algorithm is introduced in section 4.3.3.

The Krylov subspace method LSQR and the Lanczos SVD have been implemented and tested and a few results are presented in this chapter. The methods are used in chapter 4, where an efficient planar inverse acoustic method is presented and in the case study of chapter 5 (see section 5.6), where that method is applied to a large-scale experiment. 


\section{Chapter 4}

\section{Planar inverse acoustics}

\subsection{Introduction}

This chapter studies inverse acoustic problems where the source and the field are modeled as parallel planes. The techniques can be applied to vibrating plates but they can also be applied to structures that are only approximately planar using conformal mapping. This technique has been applied successfully to the front side window of a car in a wind tunnel [25]. Planar inverse acoustics can even be applied to sources that are not nearly planar but the interpretation of the results becomes more difficult. Given a source radiating sound to a number of field sensors (see figure 4.1(a)), a virtual plane can be defined between the source and the field. It is now possible to regard the virtual plane, rather than the physical source as the radiator of sound. This change of boundary conditions removes the complex source geometry from the model but it does not change the sound field above the plane. The velocities on the virtual plane can now be identified using the Rayleigh integral (see figure 4.1(b)). If the vibrations of the surface itself are necessary for further analysis,

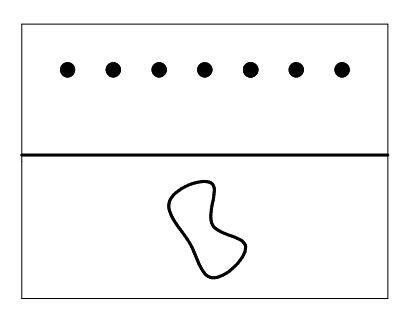

(a) source and virtual plane

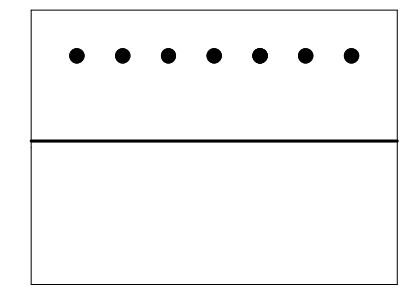

(b) reconstruction

Figure 4.1: Inverse acoustics using the Rayleigh integral 
a more general technique such as IBEM is required.

The practical importance of planar inverse acoustic methods is the fact that efficient calculation techniques are available for them such that problems which are too large for BEM can still be solved using planar techniques. Although computers become more powerful with time, the number of points that can be measured in a reasonable amount of time increases as well. Hence, efficient calculation techniques for inverse acoustic methods may well be important for many years to come.

Two planar inverse acoustic methods will be analyzed and compared in this chapter. Firstly, the well-known method termed Planar Nearfield Acoustic Holography (PNAH) is studied. It is based on the Fourier model explained in section 2.4. Although this approach leads to efficient computer calculations, it is necessary to extrapolate the field data to achieve an inverse solution which is useful in practice. Secondly, a new method is presented. It is based on the discretized form of the Rayleigh integral which has been discussed in section 2.5. A fast algorithm is proposed to solve the inverse problem. Both methods require considerably less time and computer memory than IBEM and the new method does not require any modification or extrapolation of the field data.

This chapter is built up as follows. In section 4.2 the theoretical foundation of PNAH is studied and sound field extrapolation methods are discussed. Section 4.3 proposes the new method. The methods are compared in a simulation study (section 4.4) as well as an experimental study (section 4.4). Conclusions are drawn in section 4.5.

\subsection{Planar nearfield acoustic holography}

\subsubsection{Introduction}

Planar nearfield acoustic holography (PNAH) is founded on the Fourier model of section 2.4, which describes the transfer from an infinitely large source plane to an infinitely large field plane. In practice, the field grid consists of discrete points and it has a finite area. Since the continuous Fourier transform (CFT) does not apply to this case, the CFT is approximated by the discrete Fourier transform (DFT).

PNAH consists of the following steps (see also figure 4.2). Firstly, the DFT is applied to the sound field. In practice, the sound field has been extrapolated as a pre-processing step (see section 4.2.4). Secondly, a regularized inverse solution is calculated in the wave number domain, where the forward model consists of the propagators of the Fourier model. Thirdly, the inverse DFT is applied to the inverse solution, to arrive at a spatial representation of the vibrations at the source. This approach is analogous to SVD-based regularization but the DFT takes the place 


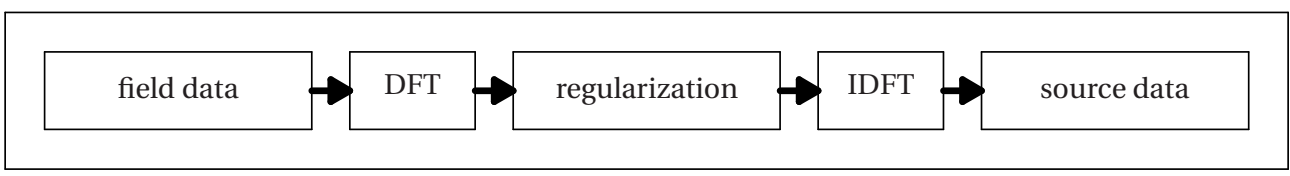

Figure 4.2: Steps involved in PNAH

of the singular vectors. The relation between the DFT and the singular vectors is explained in section 4.2.3.

To analyze quality of PNAH results, it is necessary to gain a detailed understanding of the errors that are introduced by replacing the CFT with the DFT. In the literature, the discrete model is often derived from the point of view of the wavenumber domain and the errors are explained from that point of view as well [94, 73]. This approach is common in signal processing and it is used in chapter 5 . However, section 4.2.2 derives the discrete model by introducing a set of shape functions in the spatial domain. Although the model which follows from this derivation is the same as the model which follows from the wave-number domain approach, the difference between the discrete and continuous case can be understood more easily from this point of view. Section 4.2.3 derives the relation between the DFT and the singular vectors and methods to attenuate the errors are discussed in section 4.2.4.

\subsubsection{The forward model}

To derive the discrete model mathematically, it is necessary to define a coordinate system in relation to the sensor positions. The definition is rather elaborate to simplify the equations in the wave-number domain. The measurement points are arranged in an equally spaced, rectangular grid consisting of $N_{1} \times N_{2}$ points. The length $L_{1}$ and width $L_{2}$ of the field area are the product of the number of measurement points and the distance between the sensors in each direction. This means that a row and column of sensors are missing at the right and bottom of the grid (see figure 4.3). The field points are numbered from 0 to $N-1$ in each direction and the origin has the indices $\left\{\left\lfloor N_{1} / 2\right\rfloor,\left\lfloor N_{2} / 2\right\rfloor\right\}$, where $\lfloor\cdot\rfloor$ denotes rounding downwards to the nearest integer. It follows that the coordinates of the field points are

$$
\begin{array}{rlrl}
x_{1}^{l} & =\frac{L_{1}}{N_{1}}\left(l-\left\lfloor N_{1} / 2\right\rfloor\right) & \text { where } & l \in\left\{0,1, \cdots, N_{1}-1\right\} \\
x_{2}^{m}=\frac{L_{2}}{N_{2}}\left(m-\left\lfloor N_{1} / 2\right\rfloor\right) & \text { where } & m \in\left\{0,1, \cdots, N_{2}-1\right\}
\end{array}
$$

where $x_{1}^{l}$ and $x_{2}^{m}$ are the coordinates of the field points in 1 and 2-direction respectively. The source coordinate system $\left\{y_{1}, y_{2}\right\}^{T}$ is the same as the field coordinate 


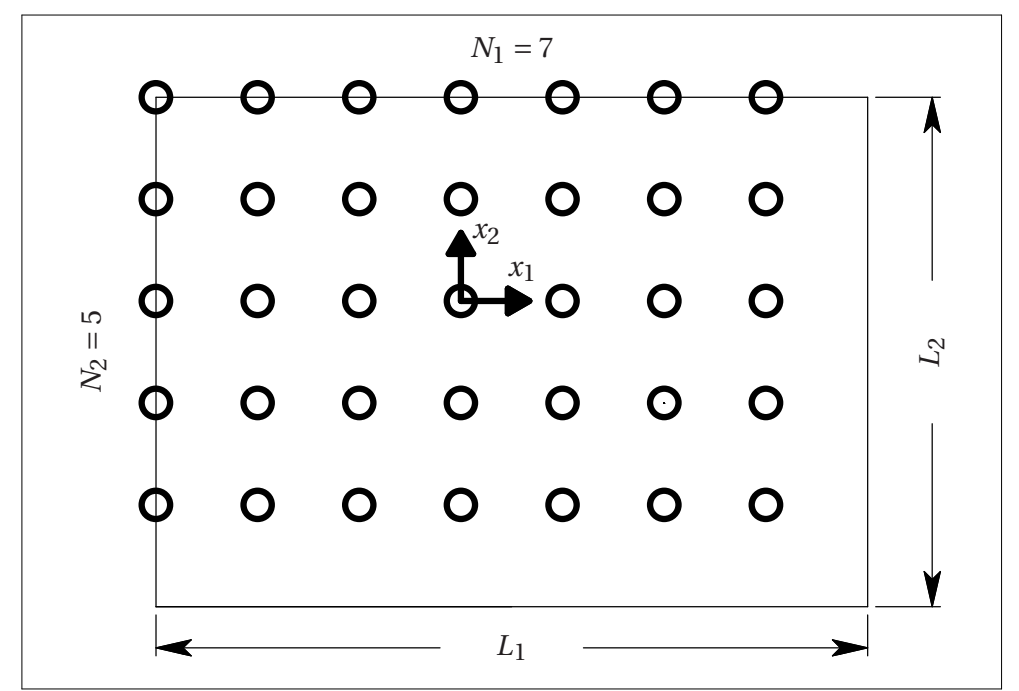

Figure 4.3: Coordinate system and definition of sizes for a rectangular field grid

system $\left\{x_{1}, x_{2}\right\}^{T}$ but there is a distance between the source and field planes in 3direction: the source plane is located at $y_{3}=0$ and the field plane is located at some distance $x_{3}$ from it. The source velocity $v(\vec{y})$ is represented by shape functions which are propagating and evanescent plane waves. The set of shape functions is chosen as follows.

$$
\begin{aligned}
v(\vec{y}) & =\sum_{i=0}^{N_{1}-1} \sum_{j=0}^{N_{2}-1} \tilde{v}_{i j} e^{-2 \pi \mathrm{i}\left(k_{1}^{i} y_{1}+k_{2}^{j} y_{2}\right) \quad \text { where }} \\
k_{1}^{i} & =\frac{1}{L_{1}}\left(i-\left\lfloor N_{1} / 2\right\rfloor\right) \\
k_{2}^{j} & =\frac{1}{L_{2}}\left(j-\left\lfloor N_{2} / 2\right\rfloor\right)
\end{aligned}
$$

where $\tilde{v}_{i j} \in \mathbb{C}$ is the contribution of the wave numbers $k_{1}^{i}$ and $k_{2}^{j}$ corresponding to direction 1 and 2 respectively. A few observations can be made. Firstly, the number of shape functions is defined to be equal to the number of measurement points. Secondly, each of the terms of the sum in equation 4.3 is periodical over a distance $L_{1}$ and $L_{2}$ in direction 1 and 2 respectively. This means that the sound field is also periodical over this distance. Errors which are caused by the fact that the model describes a periodical sound field although the actual sound field is not periodical are termed periodicity errors in this thesis.

A third observation that can be made is as follows. Since each term is a plane wave, the sound field that belongs to each term is also a plane wave with the same 
wave numbers. The sound field can therefore be calculated as (see equation 2.16):

$$
\begin{aligned}
p(\vec{x}) & =\sum_{i=0}^{N_{1}-1} \sum_{j=0}^{N_{2}-1} e^{-2 \pi \mathrm{i}\left(k_{1}^{i} x_{1}+k_{2}^{j} x_{2}\right)} \tilde{p}_{i j} \quad \text { where } \\
\tilde{p}_{i j} & =\lambda_{i j} \tilde{v}_{i j}
\end{aligned}
$$

$\lambda_{i j}$ is the propagator of the Fourier model (see also equation 2.16).

$$
\lambda_{i j}=\overline{\rho_{0} c_{0} \frac{k}{k_{3}} e^{\mathrm{i} k_{3} x_{3}}} \quad \text { with } \quad k_{3}^{i j}=\sqrt{k^{2}-\left(k_{1}^{i}\right)^{2}-\left(k_{2}^{j}\right)^{2}}
$$

Equations 4.3 through 4.8 describe the transfer from a continuous source to a continuous field exactly if the shape of vibration can be represented using the discrete wave numbers in equation 4.3. To apply the theoretical framework of regularization from chapter 3 , the equations are rewritten in terms of matrix-vector equations. The field vector contains the measured pressure at the field points. Evaluating equation 4.6 at the field points gives

$$
\begin{aligned}
p_{l m} & =p\left(\left\{\begin{array}{lll}
x_{1}^{l} & x_{2}^{m} & 0
\end{array}\right\}^{T}\right) \\
& =\sum_{i=0}^{N_{1}-1} \sum_{j=0}^{N_{2}-1} e^{-2 \pi \mathrm{i}\left(k_{1}^{i} x_{1}^{l}+k_{2}^{j} x_{2}^{m}\right)} \tilde{p}_{i j}
\end{aligned}
$$

where equation 4.10 is the discrete Fourier transform aside from the numbering of the indices. It can be represented in matrix-vector notation using the convention $p_{l m}=p_{\left(l+N_{1} m+1\right)}$ and $\tilde{p}_{i j}=\tilde{p}_{\left(i+N_{1} j+1\right)}$.

$$
\mathbf{p}=\mathbf{F} \widetilde{\mathbf{p}}
$$

The matrix F represents the two-dimensional discrete Fourier transform and it is termed the Fourier matrix. By rearranging $\tilde{v}_{i j}$ to a vector in the same way as the pressure $p_{l m}$ equation 4.7 can be expressed as

$$
\begin{aligned}
\tilde{\mathbf{p}} & =\Lambda \tilde{\mathbf{v}} \quad \text { where } \\
(\operatorname{diag}(\Lambda))_{i+N_{1} j+1} & =\lambda_{i j}
\end{aligned}
$$

where the diag operation returns a vector containing the main diagonal of a matrix and $\Lambda \in \mathbb{C}^{N_{1} \cdot N_{2} \times N_{1} \cdot N_{2}}$ is a diagonal matrix.

The source vector in the discrete spatial domain $\mathbf{v}$ is obtained using points that lie exactly underneath each field point such that the only difference is their $x_{3}$ coordinate. It follows that the spatial source vector $\mathbf{v}$ is simply

$$
\mathbf{v}=\mathbf{F} \widetilde{\mathbf{V}}
$$


The relation between the velocity at the source points and the pressure at the field points can be expressed by combining equations $4.11,4.12$ and 4.14

$$
\begin{aligned}
& \mathbf{p}=\mathbf{F} \boldsymbol{\Lambda} \mathbf{F}^{-1} \mathbf{v} \\
& \mathbf{p}=\mathbf{H}_{\mathbf{p v}}^{\mathrm{PNAH}} \mathbf{v}
\end{aligned}
$$

The framework of regularization discussed in chapter 3 can be applied directly to this problem. This framework is expressed in terms of the discrete Fourier transform in the next section.

\subsubsection{Regularization}

Since the transfer matrix of PNAH has the specific form of equation 4.15, the inverse calculation can be performed efficiently using fast Fourier transform (FFT) algorithms. It can be shown that the Fourier matrix $\mathbf{F}$ contains the eigenvectors of the transfer matrix $\mathbf{H}_{\mathrm{fs}}^{(P N A H)}$. These are orthogonal vectors, normalized to have a norm of $\sqrt{N_{1} N_{2}}$.

The SVD of this matrix is equal to its eigenvalue decomposition up to a number of details: firstly, singular values are real by definition, whereas the eigenvalues of the transfer matrix are complex. Secondly, singular vectors are scaled to have unit norm, whereas the columns of the Fourier matrix have a norm of $\sqrt{N_{1} N_{2}}$. By normalizing the vectors to unity and carrying the complex angle of the eigenvalues into the singular vectors, the following expression for the SVD is found.

$$
\begin{aligned}
\mathbf{H}_{\mathbf{p v}}^{(\mathrm{PNAH})} & =\mathbf{F} \boldsymbol{\Lambda} \mathbf{F}^{-1} \\
& =\mathbf{U} \boldsymbol{\Sigma} \mathbf{V}^{H} \quad \text { where } \quad\left\{\begin{array}{l}
\mathbf{U}=\frac{1}{\sqrt{N_{1} N_{2}}} \mathbf{F} \mathbf{\Theta} \\
\mathbf{V}=\frac{1}{\sqrt{N_{1} N_{2}}} \mathbf{F}
\end{array}\right. \\
\sigma_{i i} & =\left|\lambda_{i i}\right| \quad ; \quad \theta_{i i}=\frac{\lambda_{i i}}{\left|\lambda_{i i}\right|}
\end{aligned}
$$

Where $\Theta$ is a diagonal matrix. The singular values have not been sorted according to their value. Also note that the singular vectors of a complex matrix are defined uniquely up to a single complex angle for each pair of left and right singular vectors.

Equations 4.17 through 4.19 can be combined to the filter function $\mathscr{F}_{\alpha}\left(\sigma_{i}\right)$ of chapter 3 to arrive at the following equation

$$
\begin{aligned}
& \mathbf{H}_{\mathbf{p v}}^{\dagger \alpha\left({ }^{(\mathrm{NAH})}\right.}=\mathbf{F} \boldsymbol{\Lambda}^{\dagger \alpha} \mathbf{F}^{-1} \\
& \text { TSVD } \quad \lambda_{i i}^{\dagger \alpha}=\left\{\begin{array}{lll}
\frac{1}{\lambda_{i i}} & \text { if } & \left|\lambda_{i i}\right| \leq \alpha \\
0 & \text { if } & \left|\lambda_{i i}\right|>\alpha
\end{array}\right. \\
& \text { Tikhonov } \quad \lambda_{i i}^{\dagger \alpha}=\frac{\bar{\lambda}_{i i}}{\left|\lambda_{i i}\right|^{2}+\alpha^{2}}
\end{aligned}
$$


The discrete Fourier transforms can be carried out using FFT algorithms. PNAH is less computationally expensive than IBEM. It is faster because the transfer matrix and its SVD do not need to be calculated explicitly and because the transformation to the basis of singular vectors can be carried out using FFT algorithms. Also, it requires less memory because only the propagators need to be stored into computer memory explicitly, instead of the entire transfer matrix.

In summary, the matrices of singular vectors in PNAH are closely related to the discrete Fourier transform. SVD-based regularization can therefore take place in the discrete wave number domain. This is considerably more efficient than IBEM.

\subsubsection{Sound field extrapolation}

The main advantage of PNAH is the fact that an FFT algorithm can be used to transform the measurement data to the basis of singular vectors. This approach is only exact if the shape functions are periodical and have a period equal to the length of the field grid. Periodicity errors occur if the actual source is not periodical. A number of researchers have proposed methods to attenuate the errors. If no extrapolation is applied, PNAH extends the sound field periodically as depicted in figure 4.4(a). The inverse solution can be improved by adding fictitious field data beyond the edges of the measurement data. Although the model still has periodical shape functions, the period is increased to the combined length of the real and the fictitious data.

An intuitive approach is zero padding, where the measurement data is extended by zeros. A data-taper ${ }^{1}$ is applied to avoid discontinuities at the edges of the measurement data. This means that the measurement data are multiplied by a small value at the edges of the dataset and a value close to one at the center (see figure 4.4(b)). This approach tends to be sufficient in time-domain signal processing and it is discussed from that point of view in section 5.4. In PNAH, this approach tends to be insufficient for two reasons. Firstly, the number of points in each direction is usually in the order of magnitude of 10 , whereas the number of samples in time domain signal processing is usually in the order of magnitude of $10^{3}$ or $10^{4}$. Secondly, the results of PNAH are in the spatial domain. The edges of the calculated source velocity tend to be much smaller than they are in reality. This edge degradation is caused by the taper.

A straightforward way to extrapolate the sound field is termed border-padding which has been pioneered by Scholte and used in the current STW project [73, 74]. In this case, the dataset is extended by re-using the value at the edge for all points where the sound field is unknown. A Tukey taper is applied in such a way that all

\footnotetext{
${ }^{1}$ The term taper is used contrary to the term window to conform to the reference works [55] and [63] on spectral analysis which are used in chapter 5.
} 


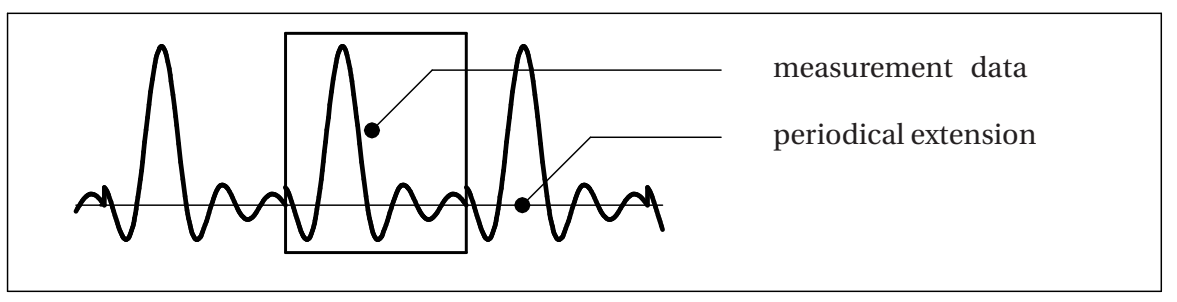

(a) Sound field and periodical extension

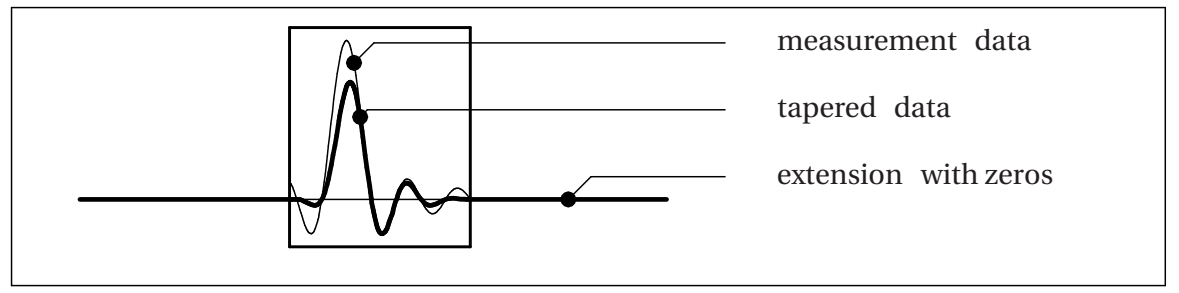

(b) Tapering and zero padding

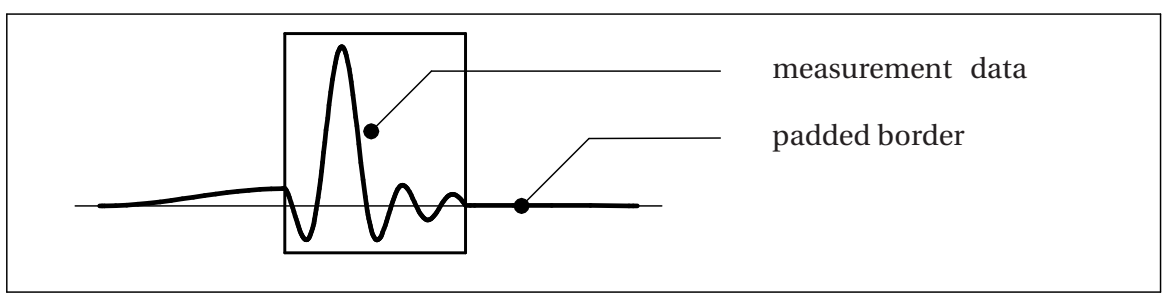

(c) Conventional border padding

Figure 4.4: Sound field extrapolation

measurement data are weighted by a factor of one and all extrapolated data are weighted by a factor smaller than one (see figure 4.4(c)). Note that this approach does not ensure the continuity of the derivatives.

A more advanced method is linear predictive border padding (LPBP), pioneered by Scholte in the current STW project [73, 74]. The Burg method is used to estimate an autoregressive (AR) system which fits the measurement data based on one row of measurement data at a time [44,32]. This filter has the form

$$
p_{l m}=\sum_{i=1}^{M} w_{i} p_{(l-i) m}
$$

Where $w_{i}, i \in\{1,2, \ldots, M\}$ denote the AR coefficients. These coefficients are used to predict the sound field at the points where no measurement data are available $\left(l>N_{1}\right)$. The sequence of field points is reversed to calculate AR coefficients which predict the sound field for $l<0$ and the same approach is also used for $m$. 
LPBP produces a high-order continuous extrapolation and the results are more accurate than zero padding or conventional border padding. Linear prediction based on the Burg method has originally been proposed for signals in the time domain and its use in the spatial domain leads to a number of theoretical peculiarities. First of all, the spatial characteristics of the sound field are assumed to be a spatially autoregressive system driven by spatial noise. In the time domain, this autoregressive system can represent a time-invariant differential equation. In the spatial domain, it means that there is a space-invariant dependence between the points, causing spatial waves. A second peculiar property is the fact that causality and stability are ensured. This means that, when predicting the values to the right of the measured sound field, the pressure at a point only depends on points to the left (the past) and not on points to the right (the future). Furthermore, vibrations tend to attenuate when going to the right (the future). Hence, the physical meaning of autoregressive systems, causality and stability is considered to be doubtful in the current context.

A final extrapolation method is based on the notion that the extrapolated sound field should comply with the acoustic equations. To achieve this, an algorithm named patch nearfield acoustic holography has been proposed by Williams and co-workers $[95,96]$. The algorithm uses the following steps. First, an inverse calculation is performed by extending the field data by zeros. Second, the sound field belonging to this source is calculated. Third, the measurement data are surrounded by the calculated sound field. Fourth, an inverse calculation is performed using the new extension of the sound field. Steps 3-4 are repeated until convergence. Promising results have been achieved. The referred author has also applied the algorithm to a model which is based on the direct spatial discretization, which is discussed in the next section, and achieved improved results. The main disadvantage of this algorithm is that the number of iterations required is so large that the required time is not competitive.

Section 4.4 demonstrates that extrapolation methods are required to achieve a practically useful Fourier-based inverse acoustic method.

\subsubsection{Summary}

Planar Nearfield Acoustic Holography is the direct application of the Fourier Acoustics to measurement data at discrete field points in the sound field. Since the forward model is analytical and the Fourier transforms can be carried out using FFT algorithms, the numerical computations require less time and computer memory than IBEM.

A disadvantage of this approach is the fact that the shape functions are fully determined by the locations of the field points. These shape functions are periodical 
which means that the method can only represent periodical sound fields. Since the sound field is rarely periodical in practice, there is an error in the forward model which leads to an error in the inverse solution. This error is termed the periodicity error in this thesis.

The periodicity errors can be reduced by means of sound field extrapolation methods but this causes extrapolation errors. The problem cannot be solved completely because the measured dataset does not fully determine the wave-number representation of the sound field.

\subsection{Toeplitz Rayleigh integral method}

\subsubsection{Introduction}

This section introduces new, fast algorithms to solve the planar inverse acoustical problem. Contrary to PNAH, the Rayleigh integral is discretized in the spatial domain. This means that the model does not contain a periodicity error such that sound field extrapolation methods are not necessary.

The approach taken is similar to a widely used method termed Statistically $\mathrm{Op}$ timised Nearfield Acoustical Holography SONAH [79, 24, 34]. Both methods use the Rayleigh integral in the spatial domain to solve the inverse problem, but there are important differences. On one hand, the new approach is more efficient, as shown in see section 4.4. On the other hand, SONAH applies to sensors which are arranged in an arbitrary pattern whereas the current method is restricted to equidistantly spaced sensors. Although nearfield source localization arrays usually consist of equidistantly spaced sensors, it does mean that the current approach does not apply directly to nearfield source localization using beamforming arrays whereas SONAH does.

A few minor differences are mentioned for completeness. Firstly, Tikhonov regularization is used in SONAH, whereas the regularization method is arbitrary for the new approach. LSQR regularization will be used for the new approach in all test cases of this chapter. Secondly, the current method uses shape functions to discretize the source vibrations whereas equivalent monopoles are used in SONAH. These differences have a negligible impact on the results.

To arrive at efficient algorithms for the current problem, it is shown that the transfer matrix has a Toeplitz structure. Because of this structure, the method is termed the Toeplitz Rayleigh Integral Method (TRIM) [103]. 


\subsubsection{The forward model}

To simplify the figures and equations, a line source is studied first and the extension to a planar source is given in section 4.3.4. The field points are assumed to be arranged on an equidistant grid and the source vector is represented using points that lie exactly underneath each point, such that the only difference is their $x_{3}$ coordinate. Some of these assumptions are abandoned in section 4.3.4, but they are necessary for a straightforward explanation.

The Rayleigh integral (equation 2.20) is discretized using arbitrary shape functions, but these shape functions are assumed to be the same for each node. The coefficients of the transfer matrix are given by

$$
\mathbf{H}_{i j}=h_{j}\left(\vec{x}_{i}\right)=\int_{S} G\left(\left|\vec{y}-\vec{x}_{i}\right|\right) N_{j}(\vec{y}) d S
$$

where $G\left(\left|\vec{y}-\vec{x}_{i}\right|\right)$ is Greens kernel in two-dimensional space and $N_{j}$ is the shape function associated with node $j$. This equation can be regarded as a discrete form of a convolution. The distances involved in the calculation of $\mathbf{H}_{32}$ and $\mathbf{H}_{43}$ as well as $\mathbf{H}_{23}$ are the same (see figure 4.5). Therefore, the transfer matrix has the following Toeplitz structure

$$
\mathbf{H}_{\mathbf{p v}}=\left[\begin{array}{ccccc}
h_{1} & h_{2} & h_{3} & \cdots & h_{n} \\
h_{2} & h_{1} & h_{2} & \cdots & h_{n-1} \\
h_{3} & h_{2} & h_{1} & \cdots & h_{n-2} \\
\vdots & \vdots & \vdots & \ddots & \vdots \\
h_{n} & h_{n-1} & h_{n-2} & \cdots & h_{1}
\end{array}\right]
$$

where $h_{i}$ is short for $h_{i}\left(\vec{x}_{1}\right)$. The same structure occurs for the case where velocities normal to the source plane are measured. If velocities parallel to the source

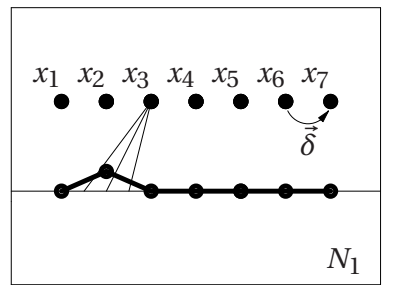

(a) Distances for $\mathbf{H}_{23}$

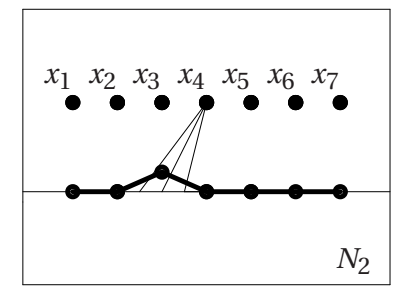

(b) Distances for $\mathbf{H}_{34}$

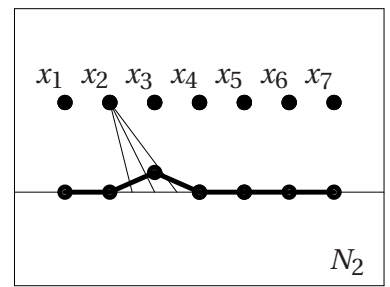

(c) Distances for $\mathbf{H}_{32}$

Figure 4.5: Graphical representation of the distances involved in three elements of the impedance matrix. Since the same distances are involved, the same value is obtained. 
plane are measured then the matrix has a similar structure but it is skew-symmetric $\left(\mathbf{H}_{i j}=-\mathbf{H}_{j i}\right)$.

Due to this Toeplitz structure, only the first column of the transfer matrix needs to be calculated and stored in computer memory. Evidently, this makes a vital difference to the computer power necessary if the problem is large. The structure is also used for faster computations.

\subsubsection{Fast evaluation of the Rayleigh integral}

This section discusses a fast matrix-vector multiplication algorithm for Toeplitz matrices. This algorithm is well known in numerical mathematics but it has not been applied in inverse acoustics. This approach is useful in regularization because a large percentage of the computational time required for Krylov subspace regularization methods consists of matrix-vector multiplications, as discussed in chapter 3.5. By improving the speed of the matrix-vector multiplications, the speed of the regularization is therefore improved as well.

The matrix-vector multiplication algorithm makes use of a circulant matrix, which is a special type of Toeplitz matrix. The transfer matrix of PNAH for a 1D source also has this structure.

$$
\mathbf{C}=\left[\begin{array}{lllll}
c_{1} & c_{N} & c_{N-1} & \cdots & c_{2} \\
c_{2} & c_{1} & c_{N} & \cdots & c_{3} \\
c_{3} & c_{2} & c_{1} & \cdots & c_{4} \\
\vdots & \vdots & \vdots & \ddots & \vdots \\
c_{N} & c_{N-1} & c_{N-2} & \cdots & c_{1}
\end{array}\right] \in \mathbb{C}^{N \times N}
$$

As in Toeplitz matrices, the matrix is structured such that $\mathbf{C}_{i j}=\hat{c}_{i-j}$ for some sequence of numbers $\hat{c}_{k}$, where $k$ can be positive or negative. Contrary to Toeplitz matrices, any point that moves out of scope on the right moves into scope on the left: $\hat{c}_{k-n}=\hat{c}_{k}$. This means that the source is periodical because a source point that lies $i$ points to the left of field point $j$, also lies $N-i$ points to the right of this field point. A sketch of the rows of this matrix is given in figure 4.6(a).

A matrix-vector multiplication with an $N \times N$ matrix commonly requires $O\left(N^{2}\right)$ operations but matrix-vector multiplication with a circulant matrix can be performed in only $O(N \log N)$ operations. All circulant matrices can be written in terms of its eigenvalue decomposition as follows

$$
\mathbf{C}=\mathbf{F} \boldsymbol{\Lambda} \mathbf{F}^{-1} \quad \text { where } \begin{cases}\operatorname{diag}(\boldsymbol{\Lambda}) & =\frac{1}{N} \mathbf{F}^{-1} \mathbf{c}_{1} \\ \mathbf{F}_{i j} & =e^{-2 \pi \mathrm{i} \frac{(i-1)(j-1)}{N}}\end{cases}
$$




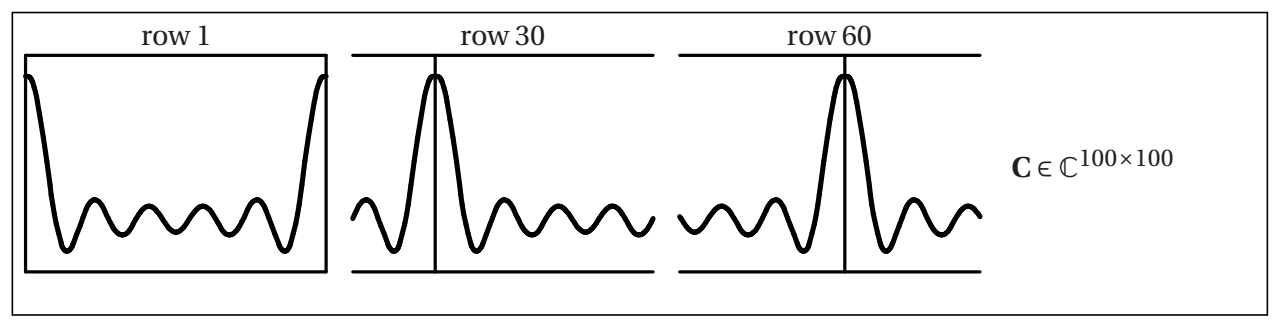

(a) Circulant transfer matrix (real part)

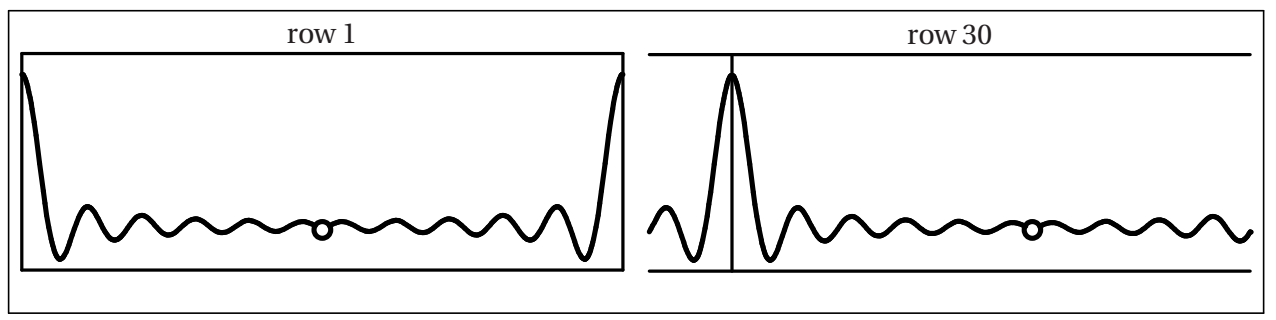

(b) Padded Toeplitz matrix (real part)

Figure 4.6: Discrete convolution

where $\mathbf{F}$ is the Fourier matrix in one dimension and $\boldsymbol{\Lambda}$ is the diagonal matrix with eigenvalues. The vector $\mathbf{c}_{1} \in \mathbb{C}^{n}$ is the first column of $\mathbf{C}$, as sketched in equation 4.25 . It can be seen that $\boldsymbol{\Lambda}$ is calculated from the first column of $\mathbf{C}$ directly.

It is emphasized that equation 4.26 is an exact algebraical identity. It does not require the DFT to converge to the CFT such that sound field extrapolation techniques are not necessary. Since fast Fourier transform (FFT) algorithms require $O(n \log n)$ calculations, multiplication with a circulant matrix can be performed efficiently by implementing the following equation.

$$
\mathbf{C x}=\mathbf{F} \boldsymbol{\Lambda} \mathbf{F}^{-1} \mathbf{x}
$$

Here, multiplication with $\mathbf{F}$ and $\mathbf{F}^{-1}$ is implemented as a fast Fourier transform and the diagonal matrix $\boldsymbol{\Lambda}$ is calculated using the first column of $\mathbf{C}$. Since only the first column of $\mathbf{C}$ is required in the calculation, the rest of the matrix does not need to be calculated or stored in computer memory. This is a dramatic reduction in memory usage.

This fast multiplication can be used for Toeplitz matrices as well. For this pur- 
pose, the Toeplitz matrix is padded to make it circulant.

$$
\begin{aligned}
\mathbf{C} & =\left[\begin{array}{cc}
\mathbf{H}_{\mathbf{p v}} & \widehat{\mathbf{H}}_{\mathbf{p v}} \\
\widehat{\mathbf{H}}_{\mathbf{p v}} & \mathbf{H}_{\mathbf{p v}}
\end{array}\right] \in \mathbb{C}^{2 n \times 2 n} \text { where } \\
\widehat{\mathbf{H}}_{\mathbf{p v}} & =\left[\begin{array}{ccccc}
0 & h_{n} & h_{n-1} & \cdots & h_{2} \\
h_{n} & 0 & h_{n} & \cdots & h_{3} \\
h_{n-1} & h_{n} & 0 & \cdots & h_{4} \\
\vdots & \vdots & \ddots & \vdots & \\
h_{2} & h_{3} & h_{4} & \cdots & 0
\end{array}\right] \in \mathbb{C}^{n \times n}
\end{aligned}
$$

Where the diagonal terms of $\widehat{\mathbf{H}}_{\mathbf{p v}}$ are undetermined. A sketch of the rows of this padded matrix is given in figure 4.6(b). The matrix-vector product $\mathbf{H}_{\mathbf{p v}} \mathbf{v}=\mathbf{p}$ can be calculated using the fast Fourier approach of equation 4.27, by writing:

$$
\left[\begin{array}{ll}
\mathbf{H}_{\mathbf{p v}} & \widehat{\mathbf{H}}_{\mathbf{p v}} \\
\widehat{\mathbf{H}}_{\mathbf{p v}} & \mathbf{H}_{\mathbf{p v}}
\end{array}\right]\left\{\begin{array}{l}
\mathbf{v} \\
0
\end{array}\right\}=\left\{\begin{array}{l}
\mathbf{p} \\
\widehat{\mathbf{p}}
\end{array}\right\}
$$

Where $\mathbf{p}$ is the required solution of the product and $\widehat{\mathbf{p}}$ is a nonphysical result that can be discarded. This extension of the matrix is well known in the mathematical literature [29] as well as the literature on signal processing [32] but the use in inverse acoustics is new to the author's knowledge.

Solving equation 4.30 for $\mathbf{v}$ is not a standard inverse problem because the righthand side contains both the known field vector $\mathbf{p}$ and the unknown vector $\widehat{\mathbf{p}}$. Furthermore, there is no attractive relation between the SVD of a Toeplitz matrix and its padded counterpart. Krylov subspace regularization methods are used instead. Section 4.3.5 considers regularization using Toeplitz matrices in more detail.

\subsubsection{Extension to practical problems}

In practice, the source is two dimensional. This case can also be handled using the matrix-vector multiplication algorithm of section 4.3.3. The source points are numbered in the same way as in PNAH (see section 4.2.2) and rearranged to have a single index. Figure 4.7(b) gives an example of the indices. The resulting transfer matrix consists of $N_{2} \times N_{2}$ blocks of dimension $N_{1} \times N_{1}$. Each of the blocks has a Toeplitz structure and the blocks themselves also form a Toeplitz structure. This structure is known as block Toeplitz with Toeplitz blocks (BTTB). Each of the blocks can be extended to form a circulant matrix and the block structure itself can be extended to form a circulant block structure such that the fully extended matrix is block circulant with circulant blocks (BCCB). The fast matrix-vector multiplication can then be performed using a 2D FFT algorithm. 


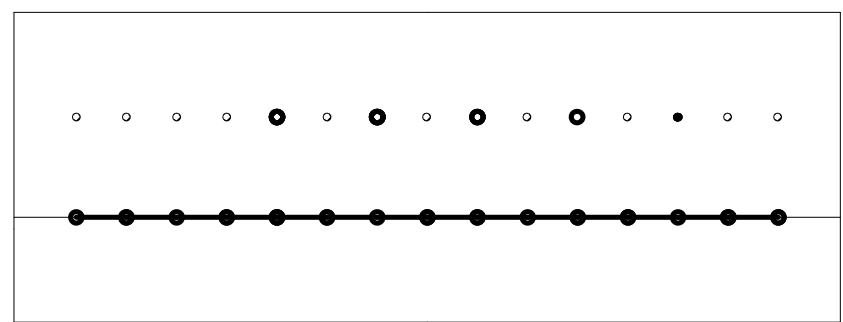

(a) field points (o), other points (.)

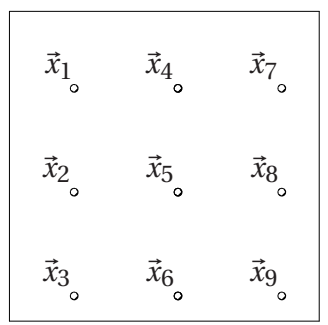

(b) grid numbering

Figure 4.7: Extension to practical problems: finer source mesh and two dimensional source

It is assumed in the previous section that a source node is located underneath each field point. This implies that the numbers of source and field nodes are equal. To reduce the discretization error in the forward model, the source points can be chosen on a grid twice as fine as the field grid. To maintain the Toeplitz structure, the field points in the model must be arranged on the same grid (see figure 4.7(a)). The algorithm for matrix-vector multiplication calculates the pressure at the fine grid of field points such that the fast matrix-vector multiplication can be used. Since the values at the points where no measurements have taken place are not relevant to the inverse calculation, these values are discarded. By choosing the source grid fine enough, the discretization error in the forward model can be reduced to an arbitrarily small value.

A different inaccuracy can be handled in the same way. The Rayleigh integral models a source in an infinite baffle, but the baffle is rarely present in acoustic experiments. This error can be alleviated by modeling the source to be slightly larger than the field. This causes the transfer matrix to be rectangular and it is treated in the same way as the case where the source grid is finer than the field grid. It is also depicted in figure 4.7(a).

\subsubsection{Regularization}

In the literature on numerical linear algebra, several fast algorithms are known to calculate the regularised inverse of a Toeplitz matrix [29, 36]. The current study focuses on the Krylov subspace methods LSQR and the Lanczos SVD (see section 3.5). Since these methods do not rely on the matrix coefficients themselves but only on matrix-vector multiplications, equation 4.30 can be used to perform these matrixvector multiplications efficiently. The methods also use a matrix-vector multiplication by the transpose of the transfer matrix, which can be implemented as a mul- 
tiplication by a cyclic matrix as follows

$$
\begin{aligned}
\mathbf{v}_{1} & =\mathbf{H}_{\mathbf{p v}}^{H} \mathbf{p} \\
\left\{\begin{array}{l}
\mathbf{v}_{1} \\
\widehat{\mathbf{v}}_{1}
\end{array}\right\} & =\left[\begin{array}{ll}
\mathbf{H}_{\mathbf{p v}} & \widehat{\mathbf{H}}_{\mathbf{p v}} \\
\widehat{\mathbf{H}}_{\mathbf{p v}} & \mathbf{H}_{\mathbf{p v}}
\end{array}\right]^{H}\left\{\begin{array}{l}
\mathbf{p} \\
0
\end{array}\right\}
\end{aligned}
$$

A number of fast algorithms for Toeplitz matrices do not belong to the class of Krylov subspace methods. In recent years, researchers in applied mathematics and image processing have developed fast regularization methods based on the displacement rank [36]. Although these methods are numerically efficient if the regularization parameter is known, the methods are not developed to solve the inverse problem for many regularization parameters.

Given the large number of articles on regularization based on Toeplitz matrices, a comparison of various regularization techniques is an attractive opportunity for research. The main goal of this chapter is to show that the Toeplitz structure is useful to calculate inverse solutions fast and accurately. For this purpose, the new method using the Krylov subspace solver LSQR is compared to methods which are known in inverse acoustics.

\subsubsection{Conclusion}

This section has proposed an inverse acoustic technique named TRIM, which is an efficient way to solve the equations of SONAH if the field points are an equidistant grid. It uses a discretization based on piecewise polynomial shape functions contrary to the plane waves used in PNAH. Sound field extrapolation methods are not necessary in this case because the model does not exhibit periodicity errors.

The approach is similar to SONAH, but the efficiency is improved by using the Toeplitz structure of the transfer matrix which occurs if the field consists of an equidistant grid. The use of this property is new in inverse acoustics to the author's knowlegde.

\subsection{Comparative study}

\subsubsection{Introduction}

TRIM has been developed to be more accurate than PNAH and faster than SONAH. The improved efficiency is especially important if the inverse calculation is performed for many frequencies and if the number source and field points is large. This section compares the speed and accuracy of various planar inverse acoustic methods. Section 4.4.2 compares the computation times and section 4.4.3 compares the accuracy of these methods. 


\subsubsection{Computation time}

The computation times are compared using the model used in the experimental validation. It is also shown that the advantages are more pronounced for grids which have a number of source points of the form $2^{n}$, where $n$ is an integer and that the difference is larger in large-scale problems than in small-scale problems.

The source which is used in this section as well as in the experimental validation is the hard disk drive which is also used for numerical examples in section 3.4. Pressure is measured at a distance of $40 \mathrm{~mm}$ from the source surface and the field grid spans a rectangular area of $160 \times 200 \mathrm{~mm}$ with a grid spacing of $10 \mathrm{~mm}$. The source grid extends $0.1 \mathrm{~m}$ beyond the edge of the source field grid at all sides and bilinear quadrilateral elements of $0.5 \times 0.5 \mathrm{~mm}$ are used such that the source grid consists of $73 \times 81=5913$ points. The distance between the source and the field is $40 \mathrm{~mm}$. Although this has no impact on the computation times, it does influence the inverse results. In the applications of PNAH, the sound field is extended to a grid of $128 \times 128$ points. The computer used to compare the computation times has a single 32bit processor which has a clock speed of $3 \mathrm{GHz}$ and it has $1 \mathrm{~GB}$ of RAM.

The time required for numerical integration is compared first. The integration scheme which is used is an adaptive Gauss-Legendre quadrature [89]. This accurate but comparatively time-intensive integration scheme requires $75 \mathrm{~ms}$ to calculate the first column of the transfer matrix. The time required to calculate all elements of the transfer matrix directly is 26.8 seconds. In PNAH, the model is analytical and it is calculated in $10 \mathrm{~ms}$.

The computation time of the PNAH methods is as follows. Patch holography is the slowest of all tested methods. Seven minutes and thirty seconds are necessary calculate the source for 90 regularization parameters, using 500 iterations of the patch holography algorithm. PNAH based on linear predictive border padding (LPBP) using an order 4 autoregressive filter requires 2.6 seconds and PNAH based on windowing and zero padding requires 2.2 seconds. In both cases, the majority of the computations are used to compute the 2D inverse Fourier transform to represent the source on a fine grid in the spatial domain. This step is necessary to compare the result to the exact solution in the region of interest, but it is not necessary if the exact solution is unknown. If this step is omitted, the computation time reduces to 0.79 and 0.43 seconds for LPBP and zero padding respectively. These are the fastest methods which have been tested.

The speed of various regularization algorithms is shown in table 4.1 (case A). The most common way to perform Tikhonov regularization for multiple regulariation paramters is by means of the SVD. The Matlab command SVD requires 10.6 seconds to decompose the transfer matrix and it is noted that the 'economy size' 
option has been used because it requires less computation time and memory. A faster way to solve the inverse acoustic problem without the fast matrix-vector multiplication is by applying LSQR to the problem directly. It requires 3.9 seconds to complete. If the fast matrix-vector multiplication is applied, the time reduces to 2.4 seconds. In this case, the computation time is approximately two-thirds the time required for the direct implementation.

As can be seen in table 4.1 (case B), the advantages of the FFT-based matrixvector multiplication are more pronounced if the source is chosen to be a grid of $64 \times 64$ points. The time required for the new method is reduced to 0.9 seconds compared to a computation time of 3.0 seconds for LSQR using the conventional matrix-vector multiplication. Hence, the new method requires less than a third of the computation time of the fastest existing method. It is also noted that the results of the $64 \times 64$ grid are as accurate as the results of the grid of $73 \times 81$ points.

The advantages of the new method become larger as the number of source and field points increases because the 2D FFT has a computation time of $O(m \log m)$ and the conventional matrix vector multiplication requires a time of $O(m n)$, where $m$ and $n$ are the number of source and field points respectively. If the source grid consists of $128 \times 128$ points and the field grid consists of $32 \times 32$ points, the new method requires approximately a sixth of the time of the existing methods at 4.4 seconds compared to 25.2 seconds for LSQR based on a conventional matrix-vector multiplication (see also table 4.1, case C). The SVD cannot be calculated in this case due to limitations in the amount of accessible computer memory. For problems of a larger scale, the transfer matrix cannot be stored in computer memory explicitly such that only the new method can be used. The time required for the new method using $256 \times 256$ source points and $64 \times 64$ field points is 25 seconds.

Given the results, it can be concluded that PNAH using zero padding and LPBP are faster than the new approach but all other methods based on the spatial discretization are slower. The new approach is especially attractive for large-scale problems, where a reduction in computation time of a factor of 6 has been ob-

\begin{tabular}{rrrr}
\hline & A & B & C \\
\hline Source grid & $73 \times 81$ & $64 \times 64$ & $128 \times 128$ \\
Field grid & $17 \times 21$ & $17 \times 21$ & $32 \times 32$ \\
\hline SVD & $10.6 \mathrm{~s}$ & $7.8 \mathrm{~s}$ & - \\
LSQR (Conventional) & $3.9 \mathrm{~s}$ & $3.0 \mathrm{~s}$ & $25.2 \mathrm{~s}$ \\
LSQR (TRIM) & $2.4 \mathrm{~s}$ & $0.9 \mathrm{~s}$ & $4.4 \mathrm{~s}$ \\
\hline
\end{tabular}

Table 4.1: Computation times of the tested regularization algorithms 
served. It has also been shown that the new approach can perform inverse calculations for problems where the transfer matrix is too large to be stored in computer memory.

\subsubsection{Accuracy}

This section compares the accuracy of the planar inverse acoustic methods using simulations and practical experiments.

In the simulation study, the sound field is calculated using a PNAH forward model in which the source is extended to a grid of $512 \times 512$ points, $1 \%$ of noise is added to the field data and the relative error of the source vector is defined to be

$$
\epsilon_{r e l}=\frac{\left\|\mathbf{s}^{\dagger \alpha}-\mathbf{s}\right\|}{\|\mathbf{s}\|}
$$

where $\mathbf{s}^{\dagger \alpha}$ and $\mathbf{s}$ are the inverse result and the exact source respectively. It is noted that only the area below the sensors is used to determine the error because the sound field extrapolation methods give large erroneous sources beyond this area. The regularization method used for the new method is LSQR and all other methods use Tikhonov regularization. The inverse result is calculated for 90 regularization parameters and the solution which has the smallest relative error is selected.

Two sources are used in the simulation study (see figure 4.8). A smooth source is used at $1075 \mathrm{~Hz}$ and a spike-shaped source is used at the higher frequency of $9668 \mathrm{~Hz}$. These frequencies are chosen because they are have large responses in the experimental study such that a comparison between the simulation results and the experimental results is straightforward. A bar-chart of the errors is depicted in figure 4.10 . At $1075 \mathrm{~Hz}$, the new method and SONAH are the most accurate, yielding errors within $8 \%$. These methods are both based on the spatial discretization. The worst result is achieved by PNAH using windowing and zero padding at an error of almost $90 \%$. All other inverse solutions look similar (see figure 4.11). For the simulation at $9668 \mathrm{~Hz}$, the new method and SONAH have a relative error of $7.3 \%$. Patch holography is close at $10.4 \%$ but PNAH using linear predictive border padding (LPBP) has an error of $32.7 \%$. This large difference is caused by the fact that the extrapolated sound field is different from the actual sound field. Erroneous osscilations occur near the bottom and the top of the calculated source and the source is over smoothed to avoid the proliferation of these errors. LPBP requires the choice of an order of the filter. An order 4 filter has been used in all simulations and experiments which are shown in this thesis. The error can be reduced to $24,3 \%$ by choosing a filter of order 1 in this case. In both cases, the source can be distinguised clearly in figure 4.11 . Hence, it is concluded that the results of 


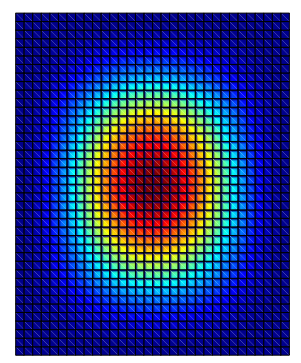

(a) Source A $1075 \mathrm{~Hz}$

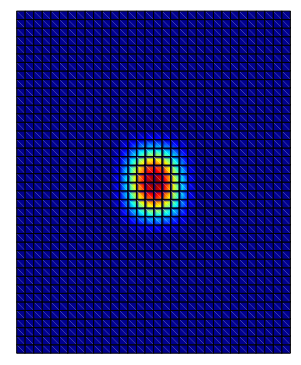

(b) Source B $9669 \mathrm{~Hz}$

Figure 4.8: Source velocities used in the simulation study (absolute value)

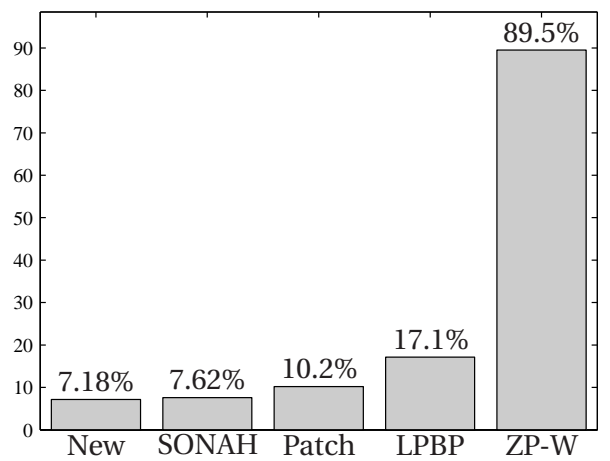

(a) Source A, $1075 \mathrm{~Hz}$

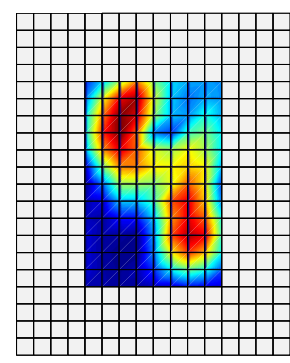

(a) $1075 \mathrm{~Hz}$

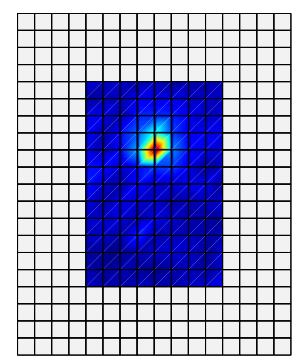

(b) $9668 \mathrm{~Hz}$

Figure 4.9: Laser vibrometer results of the experimental study (absolute value).

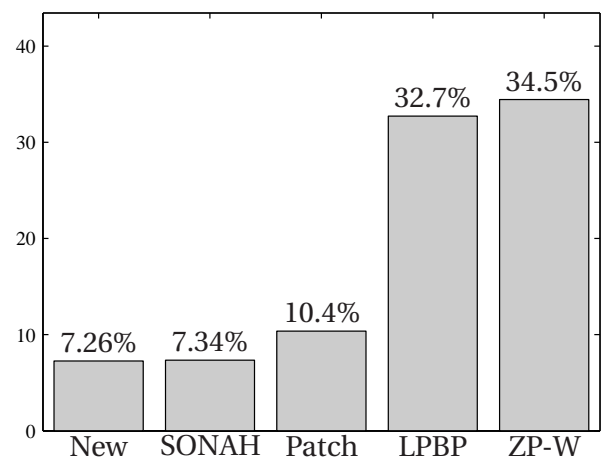

(b) Source B, $9668 \mathrm{~Hz}$

Figure 4.10: Errors which are determined in the simulation study

all methods, with the exception of windowing and zero padding, yield practically usable results.

Simulations have also been performed for various other sources and frequencies. The same trends are observed in these cases. If the vibrations at the source extend beyond the field grid, the errors are larger and the differences are small. Contrary to the results found by Williams [96], who found a large improvement in accuracy for Patch holography, the results of the current study indicate that all methods except windowing and zero padding can be the most accurate depending on the source vector and the frequency. The differences between the methods in the area below the sensors tend to be less than $5 \%$.

Measurements of a hard disk drive are used to validate the methods. The so- 
lutions are compared to laser vibrometer measurements (see figure 4.9) and the regularization parameter is chosen to be the point of maximum curvature of the L-curve [28]. Figure 4.11 depicts the results. As in the simulation study, the result of zero padding is over smoothed and all other results are similar. At $9669 \mathrm{~Hz}$, the result of linear-predictive border padding has a smaller amplitude and contains somewhat more noise than the other methods but, as in the simulation study, the point source can be seen clearly and all methods except windowing and zero padding are considered to be practically useful.

Given the results of the simulations and the experimental validation, it is concluded that the new method has shown to be robust and as accurate as SONAH for the cases which have been studied. In the same cases, PNAH using LPBP has shown to be a faster method which yields are practically useful, though less accurate, results. Although studies by several researchers will be necessary before the approach gains acceptance in the engineering community, the current section has given confidence that the method is a faster alternative to SONAH and a more accurate alternative to PNAH.

\subsection{Summary}

The planar inverse acoustic problem has been considered in this chapter. The theoretical foundation of Planar Nearfield Acoustic Holography (PNAH) has been discussed and a new approach has been proposed. The new approach yields the similar results to a widely used technique termed SONAH, but faster algorithms have been used to improve the computation time and memory. This new method is termed the Toeplitz Rayleigh Integral Method (TRIM).

It has been shown that the advantages are especially large for large-scale problems and that the new method makes it possible to perform inverse calculations for problems where the transfer matrix is too large to be stored in computer memory.

Although the new method is not as fast as planar nearfield acoustic holography (PNAH), the new method has behaved more robustly in the test cases. PNAH based on windowing and zero padding yielded results which are much less accurate than the other tested methods. The sound field extrapolation technique linear predictive border padding (LPBP) was competitive in terms of speed and it yielded results which are considered to be practically useful, but less accurate.

The new method has been implemented. The software package can determine the normal velocity as well as pressure at the source and the field data can consist of pressure, any of the three particle velocity components and combinations of these quantities. Furthermore, a number of different source norms can be used. The implemented software is used in the experimental validation of chapter 5 . 


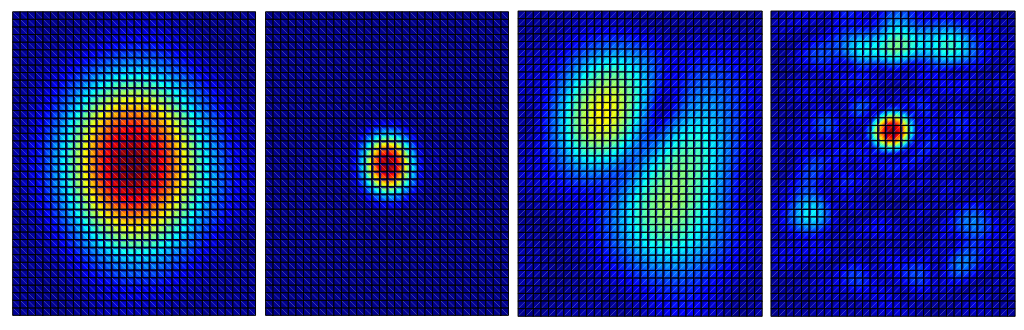

(a) inverse solutions of TRIM (absolute value)

Figure 4.11: Inverse solutions the tested methods. The results from left to right are (1) simulation of source A at $1075 \mathrm{~Hz}$, (2) simulation of source B at $9669 \mathrm{~Hz}$, (3) experiment at $1075 \mathrm{~Hz}$ (4) experiment at 9669Hz.. This figure is continued on page 59 

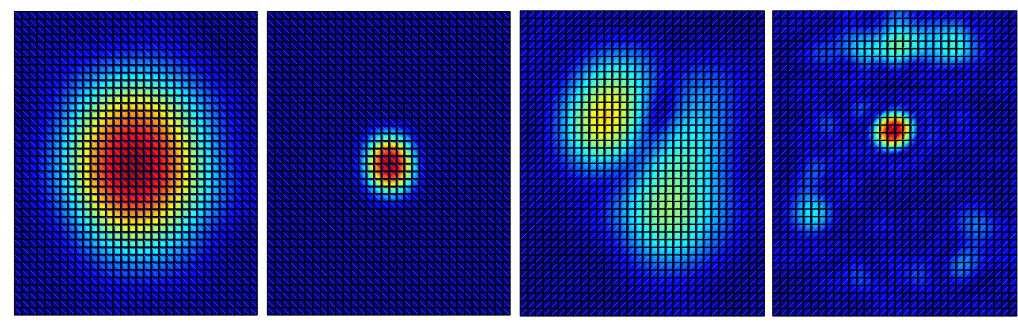

(b) inverse solutions of SONAH (absolute value)
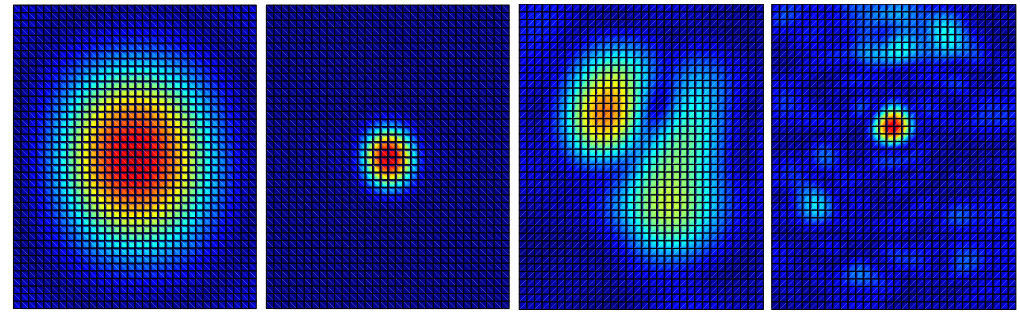

(c) inverse solutions of Patch holography (absolute value)
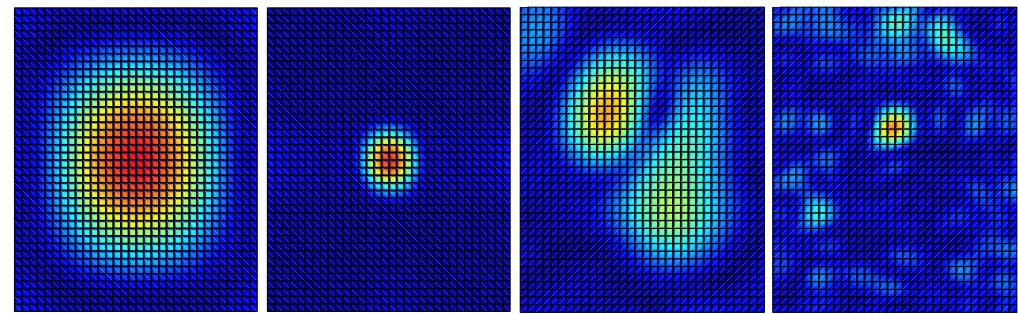

(d) inverse solutions of linear predictive border padding (order 4, absolute value)
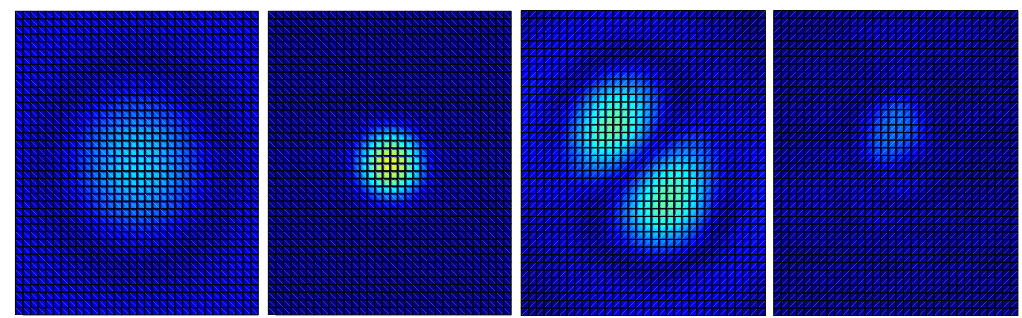

(e) inverse solutions of windowing and zero padding (absolute value)

Figure 4.11 continued 
CHAPTER 4 


\section{Chapter 5}

\section{Statistical signal processing applied to moving sensors}

\subsection{Introduction}

Signal processing techniques for moving sensors are considered in this chapter. In the literature, various time-frequency analysis methods and short-time Fourier transforms are used for this type of problem. This chapter studies the spectral estimation problem from a theoretical point of view and shows some of the fundamental limitations of spectral estimation. Tomsont's Multi-Taper method is known to be the optimal spectral estimator method within its theoretical framework. The method is compared to a number of more common alternatives using experimental data. Furthermore, the estimation of the transfer function is considered and three transfer estimators are compared.

This chapter is built up as follows. An introduction to moving sensors in inverse acoustics is given in section 5.2. The fundamentals of random vibrations are introduced in sections 5.3. Section 5.4 introduces spectral analysis techniques, including the Multi-Taper method. Based on these results, the calculation of transfer functions is discussed in section 5.5. The methods are applied to a case study in section 5.6 and a summary is given in section 5.7 .

\subsection{Moving sensors}

\subsubsection{Introduction}

Measurements for inverse acoustics are commonly array measurements, point-bypoint measurements or a combination of them. An attractive alternative is a sensor 
or array which moves through the sound field during the measurement to gather information about the sound field. Similarly, the source can be moving while the sensor or array is fixed in space. A measurement of the drive-by noise of a car is an important example of this case.

The two possible measurement setups are displayed schematically in figure 5.1. Both setups consist of an acoustic source, a field sensor which scans the sound field and a reference sensor which is fixed with respect to the source. Although the two measurement setups are quite different from a practical point of view, they are theoretically similar and the signal processing techniques used for these setups are the same. To avoid confusion, the case where the sensor moves and the source is fixed in space is considered in this chapter but the methods apply to both cases.

To calculate the source vibrations in the frequency domain based on a moving sensor, the measured time series is divided into many time series which are short enough to represent a single point in space and long enough to be able to evaluate the frequency content by applying a discrete Fourier transform. The same procedure is applied to the reference signal. The frequency content of the sound field can be calculated at each point, leading to a 2D or 3D characterization of the sound field when the measurements of all points are combined. Conventional inverse acoustic methods such as IBEM are then used to perform acoustic source localization.

To give a thorough foundation for this approach, this section discusses a literature survey and gives a new result concerning the Doppler effect.

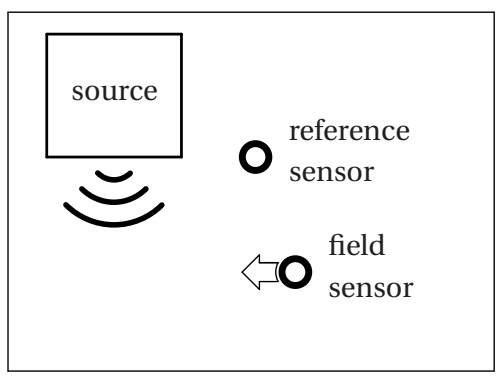

(f) moving sensor

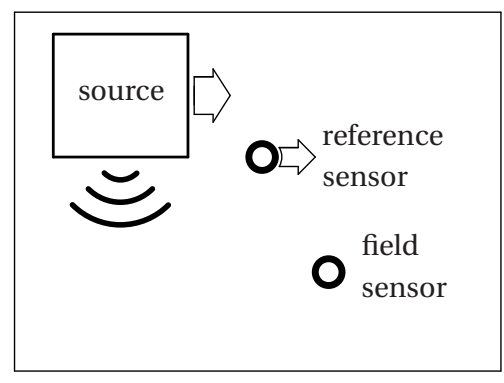

(g) moving source

Figure 5.1: schematic drawings of measurement setups

\subsubsection{Processing for moving sensors}

The main challenge in the development of inverse acoustic techniques for moving sensors is the fact that the sensor or sensors can be considered to be at a single 
point in space for a short time. This limits the achievable accuracy of the frequency domain representation of the vibrations. Several researchers have proposed solutions to this problem.

A time-domain approach has been taken by Hald [23]. A full array of sensors is used to measure a moving source and a time-domain representation of the source is depicted. The inverse acoustic calculation is performed in the frequency domain as well as the spatial wave-number domain such that the problem of calculating a Fourier transform remains equally difficult. These difficulties are discussed in detail in section 5.4.2.

An attractive alternative to the conventional short-time Fourier transform is the use of wavelets or specialized time-frequency transforms. A time-frequency transform has been used in inverse acoustics by J.C. Poisson [61], who has applied the Wigner-Ville transform to obtain a detailed time-frequency response function. This also makes it possible to perform Doppler correction in this time-frequency domain. Given the successful applications of time-frequency and wavelet methods in many applications, it is an opportunity for further research. A large body of work exists on the subjects of wavelets and time-frequency transforms (see for example $[13,11,56])$.

Time-frequency and wavelet techniques have a number of important drawbacks. Firstly, the source vibrations are best modeled to be random in an experimental environment [58]. Hence, the time-frequency content of the sound field is random as well. Since only one realization of the random time-varying vibration is available, it is not possible to calculate the statistical properties of this vibration without any further assumptions. Therefore, non-statistical definitions of the timefrequency content are often used in practice. In this chapter, it is assumed that the vibrations are stationary such that conventional statistical signal processing techniques can be used to calculate the statistical properties of the vibrations. Secondly, the definition of the Fourier transform is such that an excitation of frequency $\omega$ only has a response at $\omega$ for any linear time-invariant system. Although most timefrequency and wavelet transforms generalize the Fourier transform, they do not necessarily have this property and do not generalize this property to systems that vary in time due to the motion of the sensor.

Given these disadvantages of the time-frequency transforms and wavelets, conventional spectral analysis is considered more suitable to the problem of moving sensors in inverse acoustics. Detailed introductions to random signals and spectral analysis are given in sections 5.4 and 5.3 respectively. 


\subsubsection{The Doppler effect}

If the sensor is in motion with respect to the source, a change in frequency occurs between the source and the sensor. This phenomenon is termed the Doppler effect in this chapter even though Doppler's equation does not apply because it only applies to monopole sources. The effect has been studied in a number of articles on moving sources in inverse acoustics. Moving Frame Nearfield Acoustic Holography (MFNAH) applies the theory of PNAH (see section 2.4) to moving sources, leading to a way to deal with the Doppler effect $[41,51,52,53]$. In aeroacoustic studies, the source is modeled to be a superposition of uncorrelated point sources. Elegant equations have been derived to model the effect of air flow in a wind-tunnel as well as the effect of the rotation of a wind-turbine blade [49].

It is shown in this section that it is not useful to correct for the Doppler effect in the case study because if the Doppler effect is too large to be neglected, then the smallest bandwidth which can be resolved in signal processing is too large to be neglected as well. This result is new to the author's knowledge and it applies to many other setups where only one sensor is placed along the direction of motion.

If there is only one sensor along the direction of motion, the sensor must characterize both the spatial distribution and the frequency distribution of the sound field. If the sensor moves slowly, it can be considered to be at one place for a long time such that the frequency content can be calculated from a long time series, leading to a good frequency resolution. The Doppler shift is small in this case such that the sound field can be characterized accurately. As the velocity of the sensor increases, the frequency resolution decreases and the Doppler shift becomes larger as well. The accuracy decreases due to both of these effects. This section shows that the Doppler shift is smaller than the smallest bandwidth which can be resolved in spectral analysis, irrespective of the speed of the sensor.

The derivation uses the model of MFNAH [41]. The difference between the observed frequency $\omega^{\prime}$ and the source frequency $\omega$ can be expressed as follows. Let $v, x_{1}$ and $k_{1}$ denote the velocity of the sensor, the unit vector in the direction of motion and the wave number in $x_{1}$ direction respectively (see also section 2.4). An expression for the frequency shift is

$$
\begin{aligned}
\omega^{\prime}-\omega & =k_{1} v \\
& =\frac{2 \pi v}{\lambda_{1}} \quad \text { with } \quad \lambda_{1}=\frac{2 \pi}{k_{1}}
\end{aligned}
$$

where $\lambda_{1}$ is the wavelength in $x_{1}$ direction. The Doppler shift is compared to the bandwidth in signal processing. To formalize the statement that the sensor can be considered to be at a single point in space for a limited time, it is assumed that a constant number of $n \geq 2$ spatial samples is necessary to represent a spatial wave 
with wavelength $\lambda_{1}$. The duration $T$ of the time-series and the corresponding distance $X$ are therefore

$$
\begin{aligned}
& X=v T=\frac{\lambda_{1}}{n} \quad \text { hence } \\
& T=\frac{\lambda_{1}}{n v}
\end{aligned}
$$

In Fourier analysis, the calculated frequency content can be considered to be an average over the frequency interval $[\omega-2 \pi W, \omega+2 \pi W]$, where $W$ is termed the half-bandwidth in Hertz. As will be discussed in section 5.4.3 (equation 5.37) the time-bandwidth product is limited by some number $k \geq 1$.

$$
\begin{aligned}
2 W T & =k \\
W & =\frac{k n v}{2 \lambda_{1}}
\end{aligned}
$$

Similarly, the half-doppler shift in Hertz follows from equation 5.2.

$$
W_{\text {Doppler }}=\frac{v}{2 \lambda_{1}}
$$

Combining equations 5.6 and 5.7 gives the following equation.

$$
\frac{W_{\text {Doppler }}}{W}=\frac{1}{n k}
$$

It can be seen that the ratio of the half-Doppler shift and the half-bandwidth does not depend on the velocity of the source or on the spatial wavelength. It only depends on the signal processing parameters $k$ and $n$. Since $n \geq 2$, the largest possible value is $\frac{1}{2}$. Since the bandwidth is wider than the Doppler shift, measurements for which Doppler correction is necessary to achieve usable results have a frequency resolution which is so low that the results are unusable even after Doppler correction.

The assumptions made in this derivation apply to the measurement setup discussed in section 5.6 but they do not apply to the following cases. Firstly, the theory in the referred articles can be used if there are multiple sensors along the direction of motion. Secondly, the limitation in bandwidth given by equation 5.5 can be exceeded if the source vibrations only contain a few frequencies or if they can be modeled as autoregressive (AR) sequences or by other parametric models [55]. An important case where the current theory does apply is the case of drive-by measurements of a car or train using a vertically positioned line array. Although the setup consists of multiple sensors in this case, only one sensor is placed along the direction of motion. 


\subsubsection{Overview of the approach used in this chapter}

The signal processing approach which is outlined in this chapter has been published as a conference article by the current author [100]. An overview of the approach is as follows. The measurement data of the moving sensor and the reference sensor are divided into short time series. To characterize the relation between the field sensor and reference sensors based on these time series, the transfer function is approximated because it can be approximated more accurately from a short time series than the cross spectrum which requires a much longer time series to converge. In essence, the transfer function can be calculated by dividing the Fourier transform of the field signal by the Fourier transform of the reference signal. Since the Fourier transform requires an infinitely long function of time, the Fourier transform is replaced by the short-time Fourier transform (STFT). The multi-taper method is used which implies that the STFT is calculated several times for different tapers such that the impact of noise can be reduced by using all STFT coefficients which are available at one frequency. The transfer function is calculated based on all of the STFT coefficients using total least squares (TLS).

After an introduction to random vibrations in the next section, the multi-taper method is introduced in the context of Fourier analysis in section 5.4 and the calculation of the transfer function based on TLS is discussed in section 5.5. A case study is presented in section 5.6.

\subsection{Random vibrations}

\subsubsection{Basics}

This section introduces the basics of statistical signal processing ${ }^{1}$. A statistical approach is necessary because most vibration measurements provide different functions of time each time a measurement is performed, even if the sensor is fixed in space. Hence, it is insufficient to characterize the data in a deterministic way and a statistical approach must be used instead.

The vibrations are modeled to be a stochastic (or random) process. This is a family of random variables $\hat{S}(t)$, indexed by the time variable $t$ such that a realization of the stochastic process is a function of time. The mean $\mu_{\mathbf{s}}(t)$ and $\mu_{\mathbf{t}}(t)$ of the stochastic processes $\hat{S}(t)$ and $\hat{T}(t)$ respectively are defined to be [55]

$$
\begin{aligned}
& \mu_{\mathbf{s}}(t)=E(\hat{S}(t)) \\
& \mu_{\mathbf{t}}(t)=E(\hat{T}(t))
\end{aligned}
$$

\footnotetext{
${ }^{1}$ This section follows Percival and Walden[55] in terminology for the univariate case and Priestley [62] in the multivariate case.
} 
where $E$ denotes the expected value operator. A characterization of the relation between two stochastic processes $\hat{S}(t)$ and $\hat{T}(t)$ is as follows. The cross covariance function $R_{\mathbf{s t}}(t, \tau)$ between $\hat{S}(t)$ and $\hat{T}(t)$ is[62]

$$
R_{\mathbf{s t}}(t, \tau)=E\left(\left[\hat{S}(t)-\mu_{\mathbf{s}}(t)\right]\left[\hat{T}(t-\tau)-\mu_{\mathbf{t}}(t-\tau)\right]\right)
$$

The cross covariance function of a stochastic process with itself is termed the autocovariance function. A deterministic function can also be represented as a stochastic process. Its mean is the function itself and its auto-covariance is zero.

Many stochastic processes in acoustics are best thought of as if they continue forever. Although the vibrations vary in strength as time passes, the statistical properties are independent of time. If the mean and covariance of the stochastic processes are independent of the time instant $t$ then systems are termed second order stationary. There are other statistical properties that can be independent of time, and thus also other types of stationarity. Deterministic functions of time are generally nonstationary because their mean varies with time. The only exception is the constant function. An example of an elementary stationary stochastic process is a sine with random phase [58]

$$
\hat{S}(t)=\sin \left(\omega t+\phi_{0}\right)
$$

where $\phi_{0}$ is uniformly distributed in the interval $[-\pi, \pi]$. Stationary stochastic processes can have both random amplitude and random phase. They can be broadband as well as narrowband.

From this point on, only second order stationary stochastic processes are considered. Furthermore, the mean $\mu_{\mathbf{s}}$ and $\mu_{\mathbf{t}}$ are subtracted from the stochastic processes because this value is constant in time which implies that it is irrelevant to the study of vibration. The expression for the covariance $R_{\mathbf{s t}}(t, \tau)$ simplifies to the following expression.

$$
R_{\mathbf{s t}}(\tau)=E(\hat{S}(t) \hat{T}(t-\tau))
$$

The cross-spectral density function or cross spectrum is the frequency domain analogy to the cross covariance function. It can be shown that the Fourier transform of the cross covariance function is equal to the cross spectrum.

$$
S_{\mathbf{s t}}(\omega)=\frac{1}{2 \pi} \int_{-\infty}^{\infty} R_{\mathbf{s t}}(\tau) e^{i \omega \tau} d \tau
$$

The cross spectrum of a stochastic process with itself is the auto-spectral density function and is also known as the power spectral density function or the auto spectrum. Note that the Fourier transform does not necessarily exist in the classical 
sense. It is therefore assumed that the cross covariance function is absolutely integrable [63].

$$
\int_{-\infty}^{\infty}\left|R_{\mathbf{s t}}(\tau)\right| d \tau<\infty
$$

The realizations of the stochastic processes considered in this thesis are the pressure and the particle velocity, which are real-valued functions of time. As a consequence, the cross spectrum at $\omega$ is the complex conjugate of the cross spectrum at $-\omega$. The one-sided spectrum is therefore often used in practice. This means that the negative frequencies are ignored and the cross spectrum is multiplied by two.

In practice, the measured vibration is a single function of time such that it is not possible to observe many different realizations of the stochastic process. Hence, time averages are necessary to determine the ensemble averages such as $E(\mathbf{s}(t))$. A stochastic process is termed ergodic if this is possible. It means that the following equations hold for the stationary stochastic processes $S(t)$ and $T(t)$ [63].

$$
\begin{aligned}
\mu_{\mathbf{s}} & =\lim _{T \rightarrow \infty} \frac{1}{T} \int_{-T / 2}^{T / 2} \hat{S}(t) d t \\
R_{\mathbf{s t}}(\tau) & =\lim _{T \rightarrow \infty} \frac{1}{2 T-|\tau|} \int_{-T+|\tau| / 2}^{T-\mid \tau / 2} \mathbf{s}\left(t+\frac{\tau}{2}\right) \mathbf{t}\left(t-\frac{\tau}{2}\right) d t
\end{aligned}
$$

The right-hand sides converge with probability 1 . It can be shown that a stochastic process is mean-ergodic if and only if it has no spectral line at frequency zero. A sufficient condition for ergodicity is that the auto-correlation of a single stochastic process is zero after some finite time $a$.

$$
R_{\mathbf{s s}}(\tau)=0 \text { for }|\tau|>a
$$

It is noted that ergodicity cannot be validated in practice. It is assumed to arrive at a link between theory and experiment [55].

\subsubsection{Random vibrations in linear systems}

To describe the relation between stochastic processes at multiple source points and multiple field points, the cross spectrum is generalized to vector-valued stochastic processes, which contain scalar-valued stochastic processes as elements. In an experimental environment, the scalar-valued stochastic processes represent the outputs of a sensor such as the measured pressure or the measured particle velocity at a single point in space. In numerical modeling, they can represent the velocity at a node of a BEM model.

The stochastic processes at the source $\hat{S}(t)$ and $\hat{T}(t)$ each contain a number of scalar-valued stochastic processes as components such that they can be expressed 
as $\hat{S}(t)=\left\{\hat{S}_{1}(t), \hat{S}_{2}(t), \cdots, \hat{S}_{\hat{n}}(t)\right\}^{T}$ and $\hat{T}(t)=\left\{\hat{T}_{1}(t), \hat{T}_{2}(t), \cdots, \hat{T}_{n}(t)\right\}^{T}$. To distinguish scalar-valued and vector-valued stochastic processes, scalar-valued stochastic processes are denoted using an index from this point on: $\hat{S}_{1}(t)$. In the frequency domain, the cross spectrum between each pair of scalar-valued stochastic processes forms the cross-spectral matrix [63].

$$
\mathbf{S}_{\mathbf{s t}}=\left[\begin{array}{cccc}
S_{\mathbf{s}_{1} T_{1}} & S_{\mathbf{s}_{1} T_{2}} & \cdots & S_{\mathbf{s}_{1} T_{n}} \\
S_{\mathbf{s}_{2} T_{1}} & S_{\mathbf{s}_{2} T_{2}} & \cdots & S_{\mathbf{s}_{2} T_{n}} \\
\vdots & \vdots & \ddots & \vdots \\
S_{\mathbf{s}_{\hat{n}} T_{1}} & S_{\mathbf{s}_{\hat{n}} T_{2}} & \cdots & S_{\mathbf{s}_{\hat{n}} T_{n}}
\end{array}\right] \in \mathbb{C}^{\hat{n} \times n}
$$

The cross-spectral matrix of a vector-valued stochastic process with itself is termed the spectral matrix. To describe the transfer from one cross-spectral matrix to another, four stochastic processes are required in general. The source is represented by the vector-valued stochastic processes $\hat{S}(t)$ and $\hat{T}(t)$. They are the pressure and velocity at the source for example. The vector-valued stochastic processes in the field are denoted as $\hat{F}(t)=\left\{\hat{F}_{1}(t), \hat{F}_{2}(t), \cdots, \hat{F}_{\hat{m}}(t)\right\}$ as well as $\hat{G}(t)=$ $\left\{\hat{G}_{1}(t), \hat{G}_{2}(t), \cdots, \hat{G}_{m}(t)\right\}$. They can contain the measured pressure and particle velocity at all field points for example.

The relation between the excitations at the source and the responses in the field are expressed in the frequency domain by the transfer matrices $\mathbf{H}_{\mathbf{f s}}(\omega) \in \mathbb{C}^{\hat{m} \times \hat{n}}$ and $\mathbf{H}_{\mathbf{g t}}(\omega) \in \mathbb{C}^{m \times n}$

$$
\mathbf{H}_{\mathrm{fs}} \mathbf{s}=\mathbf{f} \quad ; \quad \mathbf{H}_{\mathrm{gt}} \mathbf{t}=\mathbf{g}
$$

where $\mathbf{s}(\omega)$ and $\mathbf{t}(\omega)$ denote the vector-valued source functions, $\mathbf{f}(\omega)$ and $\mathbf{g}(\omega)$ denote the vector-valued field functions. These equations are only meaningful if $\mathbf{s}(\omega)$ and $\mathbf{t}(\omega)$ are the Fourier transform of a function of time. Equation 5.20 holds for the Fourier transforms of the realizations of the stochastic processes $\hat{S}(t), \hat{T}(t), \hat{F}(t)$ and $\hat{G}(t)$, but this requires an extension of the theory presented here because these functions do not tend to zero as time tends to infinity such that they do not possess a Fourier transform in the classical sense $[63,50]$.

In terms of the transfer matrices of equation 5.20, the relations between the cross-spectral matrices are as follows.

$$
\begin{array}{r}
\mathbf{H}_{\mathbf{f s}} \mathbf{S}_{\mathbf{s t}}=\mathbf{S}_{\mathbf{f t}} \\
\mathbf{S}_{\mathbf{s t}} \mathbf{H}_{\mathbf{g t}}^{H}=\mathbf{S}_{\mathbf{s g}} \\
\mathbf{H}_{\mathbf{f s}} \mathbf{S}_{\mathbf{s t}} \mathbf{H}_{\mathbf{g t}}^{H}=\mathbf{S}_{\mathbf{f g}}
\end{array}
$$

Equation 5.23 is studied in detail in chapter 6 . 


\subsubsection{Signal subspace}

An important property of a vector-valued stochastic process is the number of uncorrelated sources. It is often smaller than the number of sensors and knowledge of this number and the corresponding signal subspace is useful to improve the stability of signal processing techniques and to reduce the noise.

To characterize the behavior of a system which exhibits only a few uncorrelated sources, the case of one source is considered first. Let the scalar-valued function $\mathbf{s}_{1}(\omega)$ denote the excitation of the acoustic system in the frequency domain and let the vector-valued response be denoted $\mathbf{f}_{1}(\omega)$. The relation between $\mathbf{s}_{1}(\omega)$ and $\mathbf{f}(\omega)$ is

$$
\mathbf{H}_{\mathbf{f s}_{1}} \mathbf{s}_{1}=\mathbf{f}
$$

where $\mathbf{H}_{\mathbf{f s}_{1}}(\omega) \in \mathbb{C}^{\hat{m} \times 1}$ is the transfer matrix. It can be seen that for any excitation $\mathbf{s}_{1}$, $\mathbf{f}$ is a scalar multiple of $\mathbf{H}_{\mathbf{f s}_{1}}(\omega)$. Hence, all possible responses $\mathbf{f}(\omega)$ are located on the same line in $\hat{m}$-dimensional space. This line is the signal subspace. It is one dimensional because only one source exists.

If there are $k$ uncorrelated sources which contribute to the sound field, the subspace in which all responses are located - the signal subspace - is at most $k$ dimensional. An orthogonal basis of this subspace can be determined by calculating the eigenvalue decomposition of the spectrum matrix $\mathbf{S}_{\mathrm{ff}}$. This decomposition is known as Principal Component Analysis (PCA) which is closely related to the Karhunen-Loéve transform [46].

$$
\begin{aligned}
\mathbf{S}_{\mathbf{f f}} & =\boldsymbol{\Phi} \Lambda \boldsymbol{\Phi}^{H} \\
& =\left[\begin{array}{ll}
\boldsymbol{\Phi}_{S} & \boldsymbol{\Phi}_{N}
\end{array}\right]\left[\begin{array}{ll}
\Lambda_{S} & \\
& \mathbf{0}
\end{array}\right]\left[\begin{array}{ll}
\boldsymbol{\Phi}_{S} & \boldsymbol{\Phi}_{N}
\end{array}\right]^{H}
\end{aligned}
$$

The eigenvectors are partitioned in such a way that $\boldsymbol{\Lambda}_{S}$ contains only nonzero eigenvalues. Furthermore, $\boldsymbol{\Phi}_{S}$ and $\boldsymbol{\Phi}_{N}$ are orthonormal bases of the signal subspace and the noise subspace respectively. The signal subspace is defined to be the range of the spectrum matrix in the absence of noise. PCA is a way to identify this range.

If noise is present, all eigenvalues become nonzero. If the noise is white and uncorrelated to the signal, the available spectral matrix is

$$
\mathbf{S}^{*}=\underbrace{\mathbf{S}_{\mathbf{f f}}}_{\text {signal }}+\underbrace{\sigma^{2} \mathbf{I}}_{\text {noise }}
$$

where $\sigma^{2}$ and $\mathbf{I} \in \mathbb{R}^{\hat{m} \times \hat{m}}$ are the variance of the noise and the identity matrix respectively. Substituting the principal vectors of the noiseless spectral matrix (equa- 
tion 5.25) yields

$$
\begin{aligned}
\mathbf{S}^{*} & =\boldsymbol{\Phi} \boldsymbol{\Lambda} \boldsymbol{\Phi}^{H}+\sigma^{2} \mathbf{I} \\
& =\boldsymbol{\Phi}\left(\boldsymbol{\Lambda}+\sigma^{2} \mathbf{I}\right) \boldsymbol{\Phi}^{H}
\end{aligned}
$$

which is the eigenvalue decomposition of the $S^{*}$. Hence, the signal subspace can be calculated exactly even if the noise level is unknown, provided that the noise is perfectly white and the noisy spectral matrix is known exactly. In practice, the signal subspace can be identified with remarkable accuracy if there is a considerable gap between the eigenvalues corresponding to both signal and noise and those containing only noise.

PCA can be generalized to the case where the noise is not white, if the spectral matrix of the noise is known up to a constant factor. A suitable whitening transform can be applied such that the eigenvalue problem of equation 5.25 is suitable [15].

The theory presented in this section is used throughout this thesis. Section 5.5 uses it to accurately calculate transfer functions. Chapter 6 uses it to reduce the impact of noise in cross-spectral matrices and chapter 7 considers a localization method for point sources based on the signal subspace.

\subsubsection{Summary}

This section has introduced some aspects of random vibrations. The cross-spectral matrix has been introduced and the behavior of random vibrations in linear systems has been discussed. Finally, Principal Component Analysis (PCA) has been introduced as a technique to accurately calculate the signal subspace.

To complete the link between the signal processing theory and practice, the next section considers Fourier analysis of measured signals.

\subsection{Fourier analysis}

\subsection{1 introduction}

Although the cross correlation function is a statistical average, it can be calculated from a single realization if the stochastic processes are ergodic (see section 5.3.1). To calculate the cross spectrum, the Fourier transform must be applied to either the estimated auto-correlation function or to the measured time-series.

This section considers the application of the short-time Fourier transform to a function which is known on a finite interval. The accuracy is particularly important in the case of moving sensors because the length of each time series is limited by 
the fact that the sensor can be considered to be at a single point in space for only a limited amount of time.

Three types of Fourier analysis methods can be distinguished [55]. Firstly, harmonic analysis methods are applicable to measurement data containing a so-called line-spectrum. These methods are far more accurate than other methods if this is the case but they are not applicable to general broadband signals. Secondly, parametric spectral estimation methods identify a linear system which is likely to generate the measured time series if it is driven by white noise. The spectrum of the identified linear system is then calculated as if it were truly driven by white noise. Here, the term spectrum refers to the auto spectrum. Thirdly, nonparametric spectral estimation methods estimate the spectrum using algebraic techniques which are not based on an underlying parametric model. This section considers nonparametric spectral estimation methods because they are most widely applicable.

Nonparametric spectral estimation methods are usually based on the shorttime Fourier transform (STFT), which is the tapered Fourier transform introduced in section 5.4.2 (equation 5.30). The coefficients of the STFT are not an approximation of the Fourier transform of the infinite function of time itself but the auto spectrum of a stochastic process can be approximated by performing averages over a number of STFT coefficients. Two ways of averaging are common in practice. Firstly, the auto spectrum can be estimated by averaging over the squared Fourier coefficients at a number of adjoining frequencies such that the estimated auto spectrum is the average over a frequency band. This approach is termed lag-window estimation. Secondly, if sufficient time data are available, a measured time series can be divided into a number of short time series which can overlap. The Fourier coefficients are then estimated by averaging over the squared Fourier coefficients of these separate time series. This approach is termed the Welch method (see for example [55]).

\subsubsection{Tapering}

This section introduces the theoretical basis for the use of a data taper in nonparametric spectral estimation. For this purpose, consider a deterministic function of time $\hat{s}(t)$ which has been measured during the set of time instances $\mathscr{T}$. $\mathscr{T}$ consists of discrete time instances in practice but the continuous time interval $\mathscr{T}=[-T / 2, T / 2]$ is used here. A similar approach can be used if $\mathscr{T}$ is a discrete set [81].

Only the part of $\hat{s}(t)$ which has been measured can be used to estimate the auto spectrum of a stochastic process. To arrive at a suitable approximation, the contin-

uous Fourier transform $s(\omega)$ at frequency $\omega$ is replaced by $\widetilde{s}(\omega)$ : the coefficient of 
the short-time Fourier transform (STFT). The STFT is defined as follows [11].

$$
\widetilde{s}(\omega)=\int_{\mathscr{T}} \hat{h}(t) \hat{s}(t) e^{-i \omega t} d t
$$

Here, the data taper $\hat{h}(t, \omega)$ can depend on the frequency. Note that anylinear functional which takes its input from $L_{2}(\mathscr{T})$ can be represented this way. Thus, equation 5.30 is not an arbitrary approximation of the Fourier transform, it is merely the definition that a linear relation exist between the STFT coefficient and $\hat{s}(t), t \in \mathscr{T}$. Equation 5.30 can be extended to an integral with infinite bounds as follows.

$$
\begin{aligned}
\widetilde{s}(\omega) & =\int_{-\infty}^{\infty} \hat{h}(t) \hat{s}(t) e^{-i \omega t} d t \quad \text { with } \\
\hat{h}(t) & =0 \quad \text { if } \quad t \notin \mathscr{T}
\end{aligned}
$$

The relation between $\widetilde{s}(\omega)$ and $s(\omega)$ can be found by noting that the short-time Fourier coefficient in equation 5.31 is the Fourier transform of $\hat{h}(t) \hat{s}(t)$. As a consequence of the convolution theorem, this ordinary product can be written as the convolution

$$
\widetilde{s}(\omega)=\int_{-\infty}^{\infty} \hat{h}(t) \hat{s}(t) e^{-i \omega t} d t=\frac{1}{2 \pi} \int_{-\infty}^{\infty} h(\omega-v) s(v) d v
$$

where the spectral window $h(\omega-v)$ is the Fourier transform of the taper $\hat{h}(t)$. Instead of the exact Fourier transform of $s(\omega)$, equation 5.30 yields a weighted average of $s(\omega)$ where the weight of each frequency $\omega-v$ is given by the spectral window. It follows from equation 5.33 that the solution is exact if $\hat{h}(t)=1$ such that $h(\omega)=2 \pi \delta(\omega)$ where $\delta(\omega)$ is the Dirac delta distribution. This taper has no practical use because it is not limited to the time interval $\mathscr{T}$.

There are many ways to derive a data taper based on a limited time interval $\mathscr{T}$. An interesting but unsuccessful approach is to minimize the distance between the functions $\widetilde{\mathcal{s}}(\omega)$ and $s(\omega)$ in the $L_{2}$ norm. The solution is the rectangular function $\hat{h}_{\text {rect }}(t)[99]$.

$$
\hat{h}_{\text {rect }}(t)= \begin{cases}1 & \text { if } t \in \mathscr{T} \\ 0 & \text { otherwise }\end{cases}
$$

This least squares solution suggests that the direct application of a Fourier series to the measurement data is the most accurate approximation of the Fourier transform and that any tapering diminishes the accuracy in the sense of $L_{2}$ norm. Nevertheless, a well-chosen taper does lead to more useful results. Figures 5.2 and $5.3 \mathrm{com}$ pare the rectangular function to the Hanning taper. The Fourier transformed rectangular function has a slightly higher value at $\omega-v=0$, such that the frequency $\omega$ 


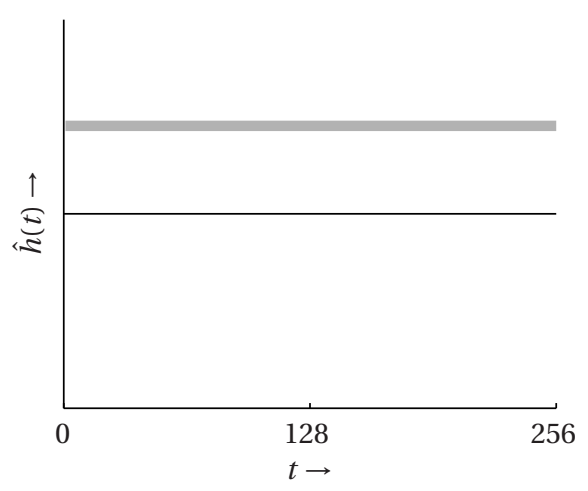

(a) taper

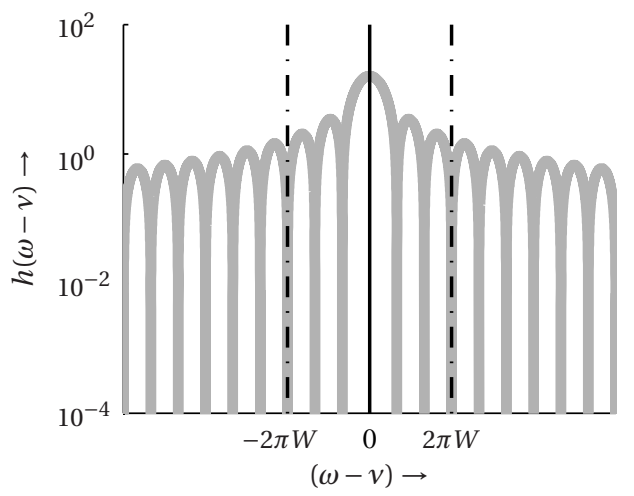

(b) spectral window

Figure 5.2: The rectangular taper. NW=3 (see section 5.4.4)

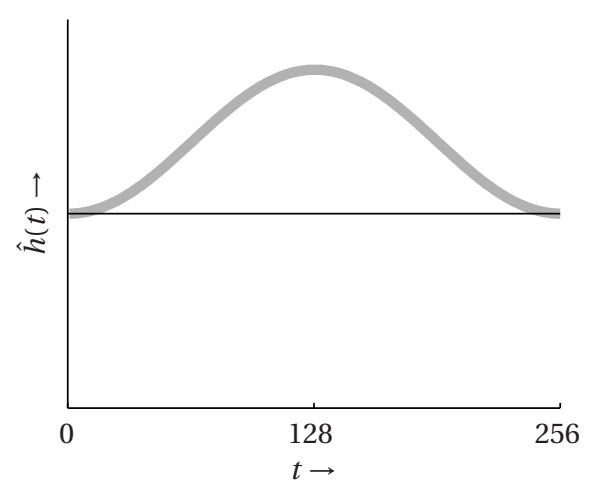

(a) taper

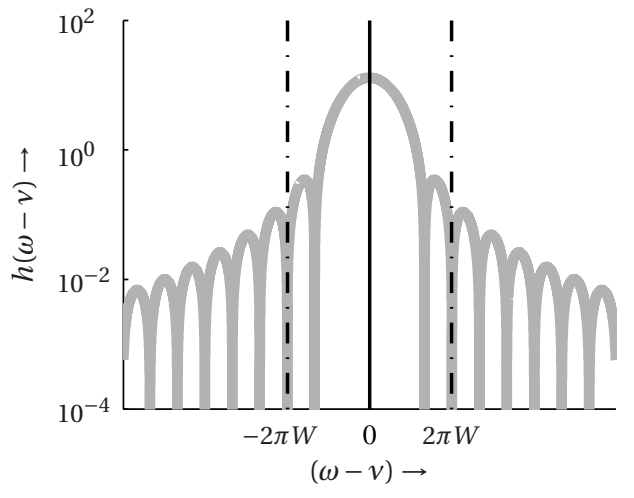

(b) spectral window

Figure 5.3: The Hanning taper. NW=3 (see section 5.4.4)

itself is weighted more heavily but the Fourier transformed Hanning taper is more concentrated in the interval close to $\omega$, denoted $\omega-v=[-2 \pi W, 2 \pi W]$ in the figure. Since this frequency interval is weighted more heavily in equation 5.33, an auto spectrum which is calculated using the Hanning taper can be interpreted as the weighted average over the frequency interval $[-2 \pi W, 2 \pi W]$. Many other tapers have similar properties. The next section derives data tapers which are optimally concentrated in this frequency interval.

It is common practice to perform a preliminary analysis of the data. The type of taper, the length of the time interval $\mathscr{T}$ and many other parameters are determined based on the measurement data and the features of the spectrum which 
must be made visible. The preliminary study can even show that a parametric method is more suitable to the data. This pragmatic approach is well-suited to the Fourier estimation problem because it deals with real data and not a mathematical model [55].

\subsubsection{Discrete prolate spheroidal sequences}

Contrary to the pragmatic approach which is common in practice, researchers at Bell labs have developed a one-for-all method for nonparametric spectral analysis named the multi-taper method [81]. The method uses multiple data tapers to calculate several Fourier estimates for each frequency even if only one time series is used. Although the Welch method is more common in structural dynamics and acoustics, it has had considerable practical success in several other fields [55].

The tapers are derived by minimizing the leakage in the following sense. The optimal taper is defined to have the largest possible 'energy' in frequency slot $\omega=$ $[-2 \pi W, 2 \pi W]$, where the half-bandwidth $W$ is chosen by the engineer based on considerations which will be discussed in section 5.4.4. To derive them, the spectral windows are denoted simply as $h(\omega)$ rather than $h(\omega-v)$ and the optimization problem is expressed as

$$
\beta(W)^{2}=\frac{\int_{-2 \pi W}^{2 \pi W}|h(\omega)|^{2} d \omega}{\int_{-\infty}^{\infty}|h(\omega)|^{2} d \omega}
$$

such that $\beta(W)=1$ if the function lies entirely inside the band and $\beta(W)=0$ if it lies outside the band. It is assumed that the desired taper is zero outside the time interval $\mathscr{T}$. It can be shown that the taper with the highest $\beta(W)$ follows from the following self-adjoint real eigenvalue problem [77, 55]

$$
\int_{\mathscr{T}} \frac{\sin \left(2 \pi W\left(t^{\prime}-t^{\prime \prime}\right)\right)}{t^{\prime}-t^{\prime \prime}} h\left(t^{\prime \prime}\right) d t^{\prime \prime}=\beta(W)^{2} h\left(t^{\prime}\right)
$$

This problem does not yield one but infinitely many orthogonal eigenfunctions. They are termed prolete spheroidal functions. The first prolete spheroidal function is the most bandlimited function in terms of equation 5.35, the second is the most bandlimited function which is orthogonal to the first, etcetera. If these functions are used as tapers in Fourier analysis the higher tapers pick up 'information' that is lost by using just the first prolate spheroidal function alone. As a result, the higher tapers can even be concentrated at the edges of the time interval, instead of the center. 
A related eigenvalue problem exists for the practical case where the continuous time interval $\mathscr{T}$ is replaced with the discrete set $\{1,2, \cdots, N\}$ and the bandwidth $W$ is denoted in cycles per sample. The eigenvectors are called discrete prolate spheroidal sequences (DPSS) or Slepians. These vectors are depicted in figures 5.4, 5.5 and 5.6.

Although there are infinitely many eigenfunctions in the continuous time problem, only a few are useful for spectral estimation, because the first few eigenfunctions are concentrated inside the frequency interval $[-2 \pi W, 2 \pi W]$ and the other eigenfunctions are concentrated outside this interval (see for example figure 5.6). The number of tapers which are concentrated inside the interval can be approximated by a straightforward equation.

The eigenvalues $\beta_{k}(W)$ specify the relative amount of 'energy' inside the frequency interval in accordance with equation 5.35. It can be shown that these eigenvalues exhibit a sharp drop from approximately unity to nearly zero at a value $k$, (the Shannon number) in both the discrete and the continuous problem (see also figure 5.7) [55]

$$
k=2 W T
$$

where $\omega=[-W, W]$ such that $W$ is the half-bandwidth. The time interval is $\mathscr{T}=$ $[-T / 2, T / 2]$. This equation can be interpreted as follows. Only the first $k$ vectors are used for Fourier analysis because the other DPSS vectors cause leakage. Thus, equation 5.37 expresses a fundamental limitation of the number of tapers which can be used for the bandwidth and the time. The consequences of equation 5.37 and its relation to the famous Heisenberg relation have caused considerable discussion in the literature. The reader is referred to Slepian [76] for more information.

\subsubsection{The multi-taper method in practice}

The main practical advantage of the multi-taper method compared to the Welch method is the fact that several orthogonal tapers are available for a single time series. Hence, the time-series does not have to be divided into shorter time series to perform averages 5.8. From that point of view, the method is similar to lag-window estimation, where the spectrum is determined by taking a moving average multiple frequencies. Since the DPSSs are optimized to avoid leakage, the multi-taper method has a higher dynamic range.

The multi-taper method has two parameters: the number of samples of a time series $N$ and the discrete bandwidth-time product $N W$. In the case of moving sensors in inverse acoustics, the number of samples is limited by the time a sensor can 


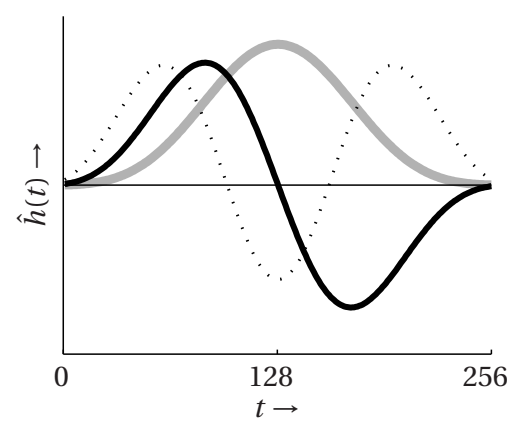

(a) taper

Figure 5.4: DPSS $(\mathrm{NW}=3)$. First

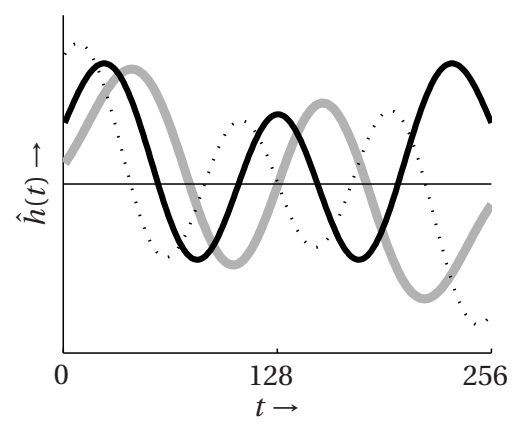

(a) taper

Figure 5.5: DPSS $(\mathrm{NW}=3)$. Fourth

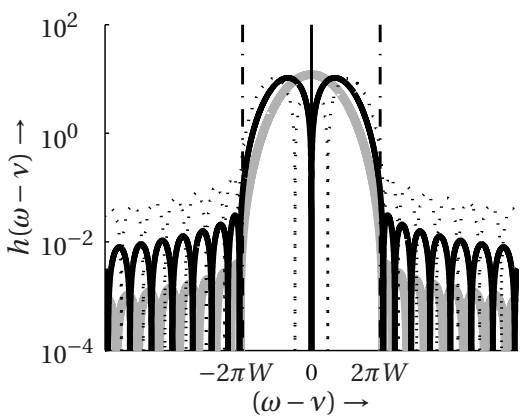

(b) spectral window

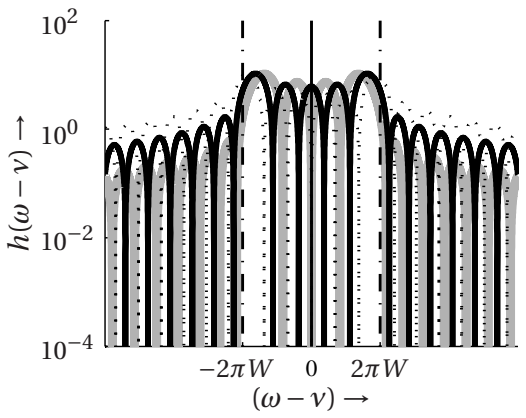

(b) spectral window ), Fifth $(-)$, sixth $(\cdots)$

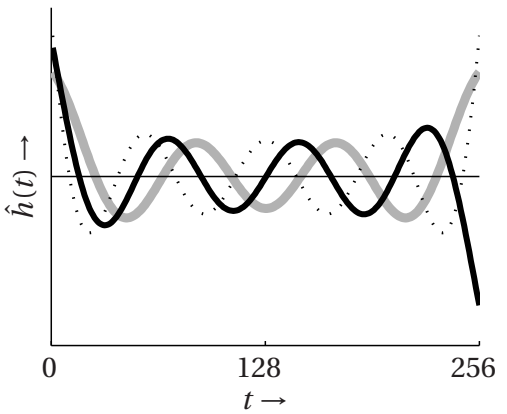

(a) taper

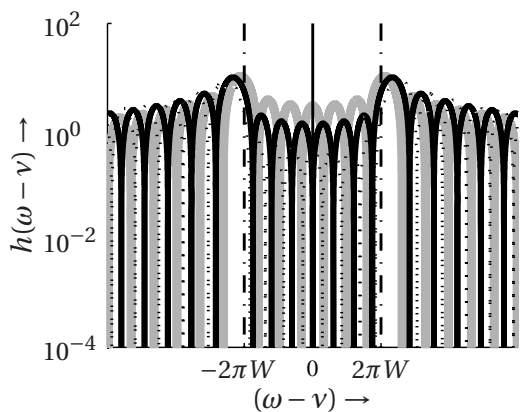

(b) spectral window

Figure 5.6: DPSS $(\mathrm{NW}=3)$. Seventh $(\square)$, Eighth $(\square)$, Ninth $(\ldots \ldots)$ 


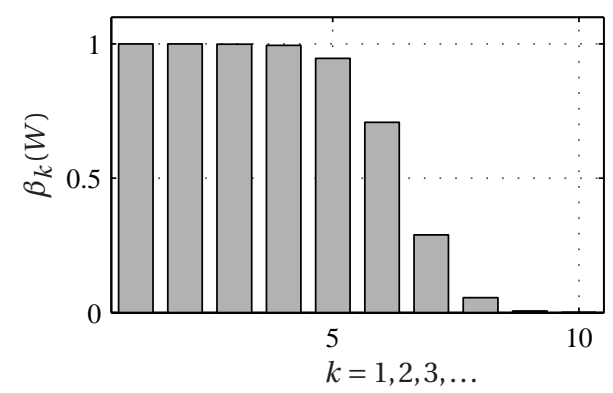

Figure 5.7: First ten eigenvalues $\beta_{k}(W)$ for $\mathrm{NW}=3$

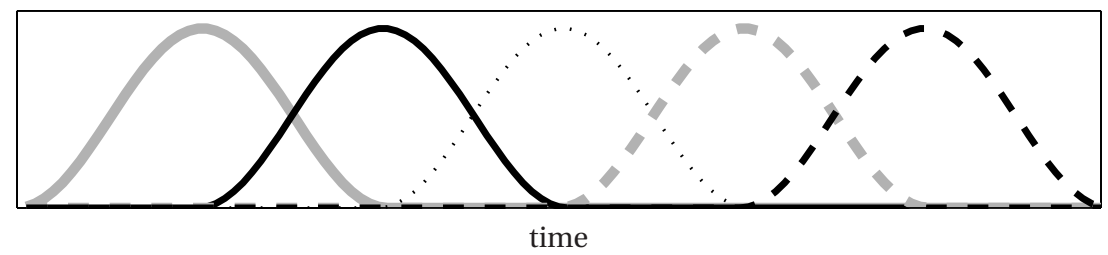

(a) Welch method using five tapers

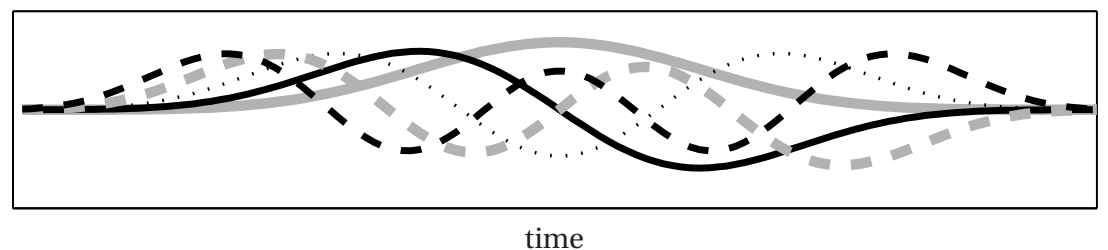

(b) multi-taper method using five tapers $(\mathrm{NW}=20)$

Figure 5.8: Tapers in the Welch method and the multi-taper method

be considered to be at the same place. In other applications, it is ultimately limited by the processing power of the signal processing hardware. The choice of the bandwidth $W$ is a trade-off between the number of tapers which can be used for averaging and the ability to determine the local features of the spectrum. It therefore depends on the features that must be made visible. In practice, values of $N W=2,3$ or 4 are common, but any real positive number can be used. Since the bandwidth is usually chosen to be so wide that the frequency bands at different frequency bins overlap, the estimated Fourier coefficients at different frequencies are dependent. However, the estimated Fourier coefficients at a single frequency are uncorrelated if the spectrum is constant in the frequency band [55].

The first few estimated Fourier coefficients have much less leakage than the higher coefficients. To achieve a spectral estimate with as little leakage as possi- 
ble, this knowledge must be taken into account. The adaptive multi-taper spectral estimator defined by Percival and Walden [55] calculates such a weighted average based on the eigenvalues $\beta_{k}$ as well as the spectral properties of the measured time series itself. Although the weighting is not discussed any further, it is used in all practical test cases of this chapter and it contributes substantially to the accuracy of the method.

\subsubsection{Summary}

The Fourier transform of a function cannot be calculated exactly in practice because measurements have a finite length. To arrive at an estimated auto spectrum, nonparametric spectral estimation methods apply a data taper to the measured time series to calculate the short-time Fourier transform. An approximation of the auto spectrum can be calculated by averaging over many short-time Fourier coefficients.

The multi-taper method uses multiple data tapers to arrive at an estimate of the average auto spectrum in a frequency band. The tapers are derived to minimize leakage. The multi-taper method is especially useful in the case of moving sensors because this approach yields multiple Fourier coefficients for each frequency without the need to average over multiple time series.

\subsection{Transfer function estimation}

\subsubsection{Introduction}

The fact that the field sensor can be considered to be at a single place for a short time has two consequences. The first consequence is the fact that Fourier analysis must be applied to signals which can be considered to be stationary for only a short time, as discussed in the previous section. This section considers the second consequence, which is the fact that the source amplitude in a frequency band can vary considerably over time. Figure 5.9 illustrates this behavior. It depicts the displacement of a weakly damped mass-spring-damper system driven by bandlimited white noise. The vibration level varies strongly during the simulation and the rate of change is quite slow: it takes dozens of wavelengths to go from a maximum to a minimum. Due to the strength variation, cross-spectral estimates require a long time series to converge.

Transfer function estimates are more attractive because they can be calculated exactly based on a single realization of the stochastic processes of the source and the field if noise is absent. From a mathematical point of view, this can be shown as 


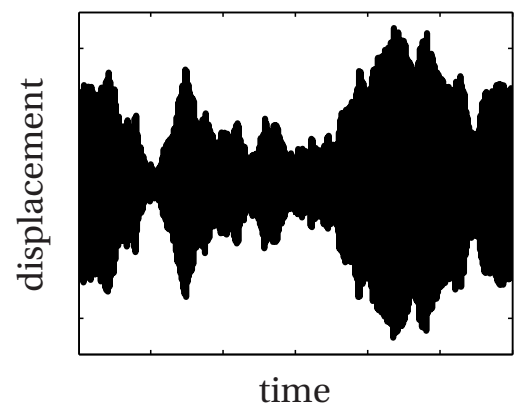

Figure 5.9: Strength variations in a stationary stochastic process

follows. Let the (deterministinc) functions $\mathbf{f}_{1}(\omega)$ and $\mathbf{g}_{1}(\omega)$ be the response to a single unknown deterministic source function $\mathbf{s}_{1}(\omega)$ and assume that there is no noise or leakage. It follows that the transfer function from $\mathbf{f}_{1}$ to $\mathbf{g}_{1}$ is independent of the source function, such that it is certainly independent of source strength variations.

$$
\begin{aligned}
h_{\mathbf{g}_{1} \mathbf{f}_{1}} \mathbf{f}_{1} & =\mathbf{g}_{1} \quad \text { where } \quad h_{\mathbf{g}_{1} \mathbf{f}_{1}}=\frac{h_{\mathbf{g}_{1} \mathbf{s}_{1}}}{h_{\mathbf{f}_{1} \mathbf{s}_{1}}} \\
h_{\mathbf{g}_{1} \mathbf{f}_{1}} & =\frac{\mathbf{g}_{1}}{\mathbf{f}_{1}}
\end{aligned}
$$

The transfer function $h_{\mathbf{g}_{1} \mathbf{f}_{1}}$ characterizes the sound field and it will therefore be approximated. It can be shown that the same transfer function $h_{\mathbf{g}_{1} \mathbf{f}_{1}}$ also describes the relation between the realizations of the stochastic processes $\hat{F}(t)$ and $\hat{G}(t)$ in the frequency domain if they are caused by the stochastic process $\hat{S}(t)$ at the source. In practice, the $\mathbf{f}$ and $\mathbf{g}$ are replaced by the STFT coefficients $\tilde{\mathbf{f}}$ and $\tilde{\mathbf{g}}$ respectively. This yields an approximation of the transfer function [63].

Equation 5.39 is especially useful in the case of moving sensors if $f_{i}(\omega)$ is a reference sensor which is fixed in space. Since this sensor receives the same stationary stochastic process irrespective of the position of the field sensor, the auto spectrum at the reference sensor can be calculated using a large number of averages. The auto spectrum at the field sensors can therefore be calculated accurately as a post-processing step from equation 5.23

$$
s_{g_{1} g_{1}}=\left|h_{g_{1} f_{1}}\right|^{2} s_{f_{1} f_{1}}
$$

where $h_{\mathbf{g}_{1} \mathbf{f}_{1}}$ is calculated from equation 5.39. The main challenge of this approach is to accurately calculate the transfer function, which is discussed in the next section.

\subsubsection{Total least squares}

In the case of the multi-taper method, multiple STFT coefficients are available to calculate the transfer function. Since equation 5.38 describes a linear relation be- 


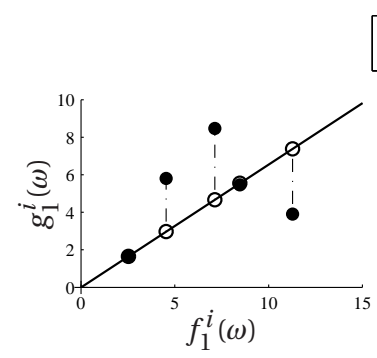

(a) Vertical fit

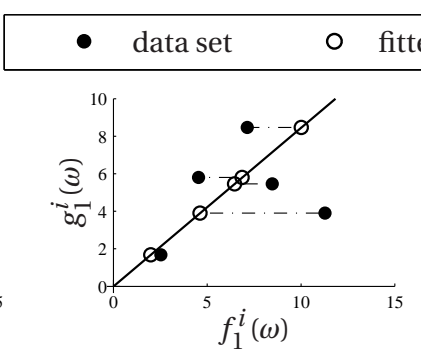

(b) Horizontal fit

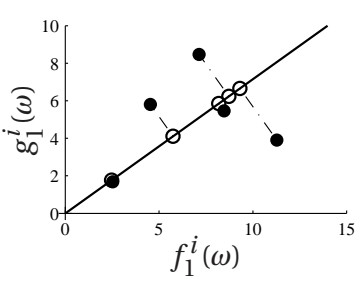

(c) Total least squares

Figure 5.10: Three least squares methods applied to the same data set. To arrive at a 2D graph, the variables are taken to be real although they are complex in practice.

tween the STFT coefficients of the field sensor and the reference sensor, identifying a transfer function is equivalent to fitting a line through the origin, close to a number of points. The line is the one-dimensional signal subspace (see section 5.3.3).

The line can be fitted in several ways. The conventional least squares fit minimizes the squared distance in vertical direction (see figure 5.10(a)). The mathematical expression for this minimization is as follows. Let $\tilde{f}_{1}^{i}(\omega)$ and $\tilde{g}_{1}^{i}(\omega)$ where $i \in\{1,2, \cdots, l\}$ denote the STFT coefficients of the field sensor and the reference sensor respectively, then the conventional least squares problem can be expressed as the following problem

$$
h_{\text {vertical }}=\underset{h}{\operatorname{argmin}} \sum_{i=1}^{l}\left|\tilde{\mathbf{g}}_{1}^{i}-\widehat{\mathbf{g}}_{1}^{i}\right|^{2} \quad \text { with } \quad \widehat{\mathbf{g}}_{1}^{i}=h \tilde{\mathbf{f}}_{1}^{i}
$$

which can be solved for $h$ as a conventional least squares problem. The estimator is well founded if there is no noise in horizontal direction and if the statistical properties of the noise are as follows. The noise at all STFT coefficients is normally distributed, uncorrelated and have the same variance [93]. Similarly, the horizontal least squares fit is suitable if there is no noise in vertical direction (see figure 5.10(b)). In analogy to equation 5.41, it can be expressed as follows

$$
h_{\text {horizontal }}=\arg \min _{h} \sum_{i=1}^{l}\left|\tilde{\mathbf{f}}_{1}^{i}-\widehat{\mathbf{f}}_{1}^{i}\right|^{2} \quad \text { with } \quad h \widehat{\mathbf{f}}_{1}^{i}=\tilde{\mathbf{g}}_{1}^{i}
$$

This equation can be solved for $1 / h$ as a conventional least squares problem. The total least squares fit minimizes the squared, perpendicular distance between the points and the line. It is suitable if the noise in horizontal and vertical directions have the same variance. It can be expressed as

$$
h_{\text {total }}=\arg \min _{h} \sum_{i=1}^{l}\left|\tilde{\mathbf{f}}_{1}^{i}-\widehat{\mathbf{f}}_{1}^{i}\right|^{2}+\left|\tilde{\mathbf{g}}_{1}^{i}-\widehat{\mathbf{g}}_{1}^{i}\right|^{2} \quad \text { with } \quad h \widehat{\mathbf{f}}_{1}^{i}=\widehat{\mathbf{g}}_{1}^{i}
$$


This is the total least sqares estimator [19], which is also known as the $H_{v}$ estimator. It is the Maximum likelihood estimator for Gaussian white noise on both signals. Hence, it has a number of attractive statistical properties which the other estimators do not have [93]. The total least squares problem can be solved as a singular value problem. In appendix $B$, it is shown that it is equivalent to calculating the signal space using a spectral matrix which is calculated by averaging (see also [64]).

If the cross-spectral matrix could be calculated exactly, then the solution of TLS would be exact even if white noise is present. To show this, consider the vectorvalued stochastic proces $\hat{V}(t)=\{\hat{F}(t), \hat{G}(t)\}$. The eigenvalue decomposition of the spectral matrix $\mathbf{S}_{\mathbf{v v}}$ is denoted as

$$
\mathbf{S}_{\mathbf{v v}}=\left[\begin{array}{ll}
\phi_{f_{1} 1} & \phi_{f_{1} 2} \\
\phi_{g_{1} 1} & \phi_{g_{1} 2}
\end{array}\right]\left[\begin{array}{ll}
\lambda_{1}+\sigma^{2} & \\
& \sigma^{2}
\end{array}\right]\left[\begin{array}{ll}
\phi_{f_{1} 1} & \phi_{f_{1} 2} \\
\phi_{g_{1} 1} & \phi_{g_{1} 2}
\end{array}\right]^{H}
$$

where the first eigenvector spans the signal space exactly and the second eigenvector spans the noise space exactly. It follows that the transfer function can be calculated as follows.

$$
h_{g_{1} f_{1}}=\frac{g_{1}^{i}}{f_{1}^{i}}=\frac{\phi_{g_{1}}}{\phi_{f_{1}}}
$$

The total least squares problem is implemented as equations 5.44 and 5.45 where the cross-spectral matrix is calculated by averaging. The source strength variations cause the eigenvalues to be inaccurate but the eigenvectors are still calculated accurately. Since two reference sensors are used in the case study, the case of multiple reference sensors and field sensors has been implemented. This case is also considered in appendix B.

\subsubsection{Summary}

This section has shown three ways to calculate transfer functions based on measurement data. The total least squares estimator is an asymptotically unbiased estimator if white noise is present at both sensors. The case study in the next section compares transfer estimation based on total least squares to conventional least squares.

\subsection{Experimental validation using moving sensors}

\subsubsection{Introduction}

In this section, The introduced signal processing method is applied to a case study 


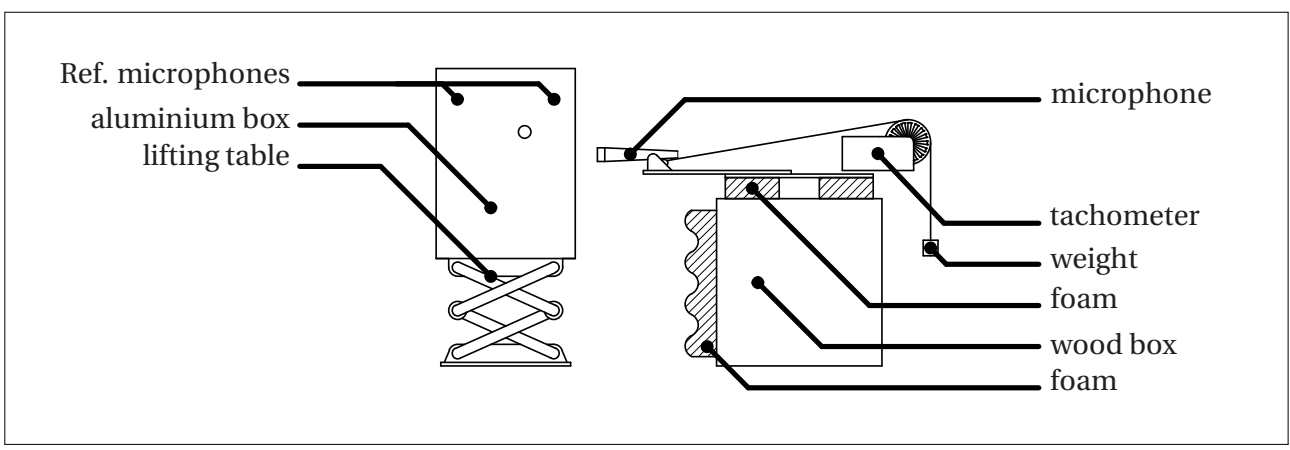

Figure 5.11: schematic view of a measurement setup

involving moving sensors. A schematic overview of the measurement setup is given in figure 5.11. An important aspect is the choice of the source. It is a loudspeaker in a stiff aluminium box with a circular hole. The hole diameter is $20 \mathrm{~mm}$ such that the velocity at the source is nonsmooth. In previous research of the group, the first few dynamic modes of a thin metal plate have were used as a noise source. However, such smooth shapes are easier to reconstruct accurately, making it difficult to assess the quality of the inverse techniques based on these shapes. Hence, the current source should be seen as a worst case for engineering purposes in the context of source localization for arbitrary sources.

The field microphone is located $10 \mathrm{~mm}$ from the source surface and it is connected to rails such that it can move horizontally, parallel to the source, but it is constrained in the other directions. To achieve a 2D representation of the sound field, the measurement is performed at 22 different heights which are $5 \mathrm{~mm}$ apart.

The sensor is moved by hand and a tachometer is used to measure the horizontal location of the microphone. A wire connects the microphone to the tachometer wheel, and a resolution of $1 \frac{1}{6} \mathrm{~mm}$ per tachometer tick is achieved. Finally, foam is used to attenuate reflections from the wood box.

\subsubsection{Results}

The source emits white noise and two reference microphones are placed at a fixed position with respect to the source. The results are analyzed for the frequency of $10 \mathrm{kHz}$ and the frequency band in the multi-taper method is chosen to be $100 \mathrm{~Hz}$ such that $2 W T=24$. For each horizontal line, the measurement time is 3 seconds or less, depending on the measurement, leading to 150000 samples of field data at the sampling rate of $51.2 \mathrm{kHz}$. This data is divided into time series with an overlap of $80 \%$, which leads to 370 time series of 2048 samples for each horizontal line. 


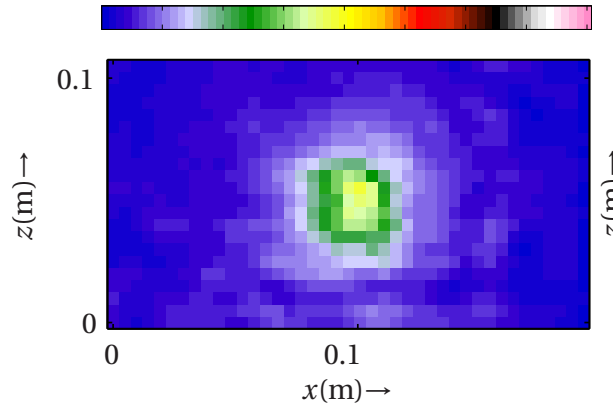

(a) MTM, TLS (absolute value)

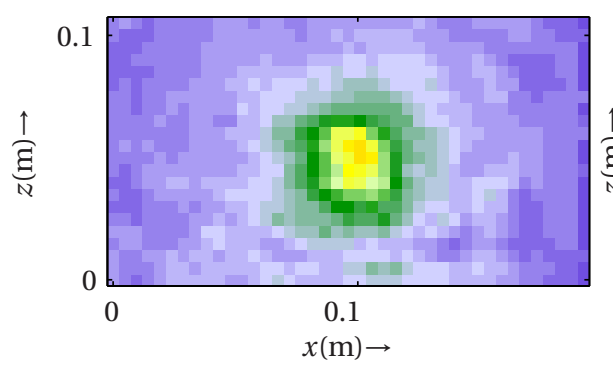

(c) Welch, TLS (absolute value)

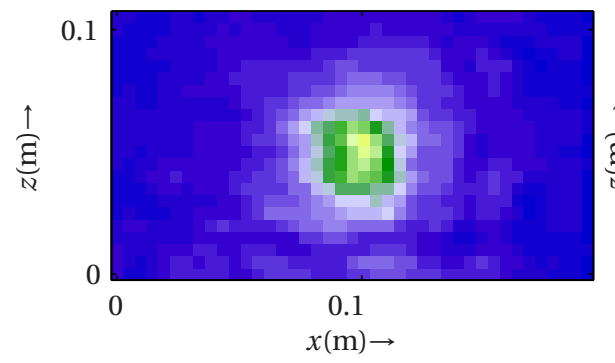

(e) least squares (absolute value)

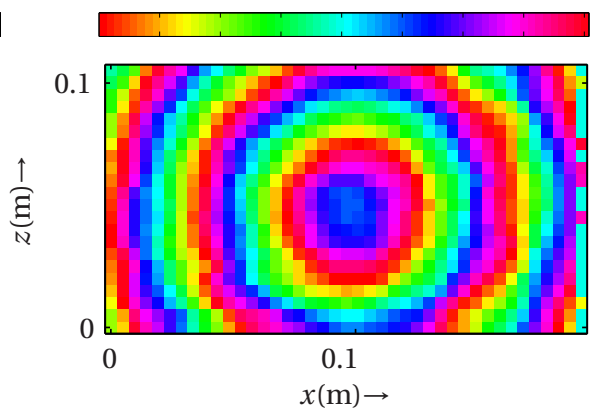

(b) MTM, TLS (phase)

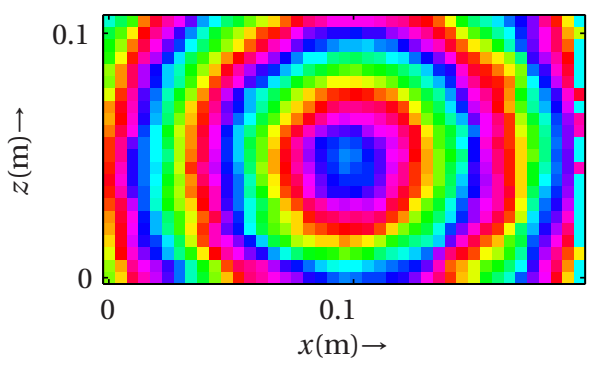

(d) Welch, TLS (phase)

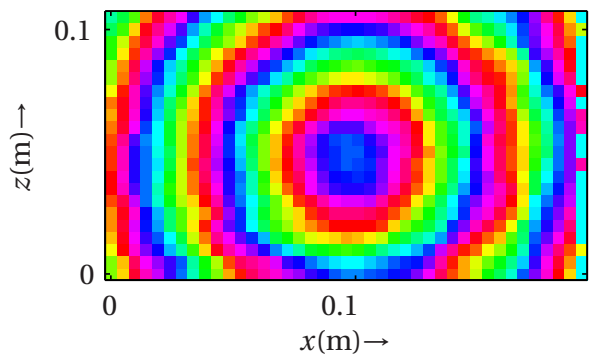

(f) least squares (phase)

Figure 5.12: Comparison between cross-spectral and transfer estimates 


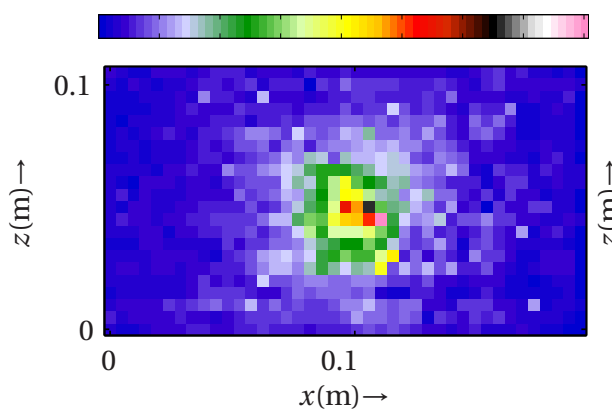

(g) one taper (absolute value)

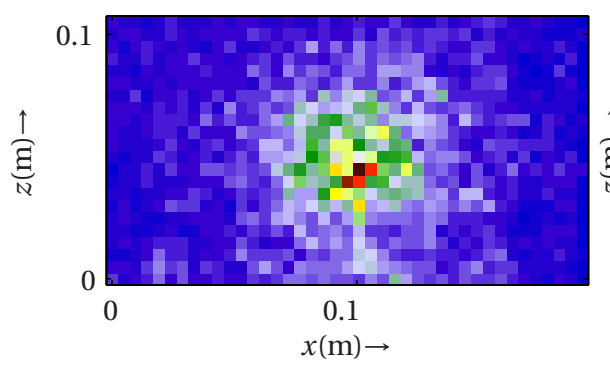

(i) cross spectrum (absolute value)

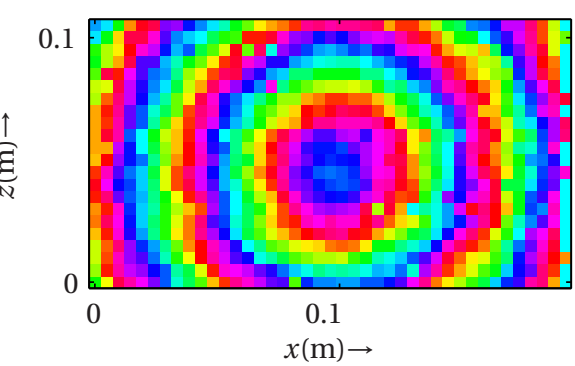

(h) one taper (phase)

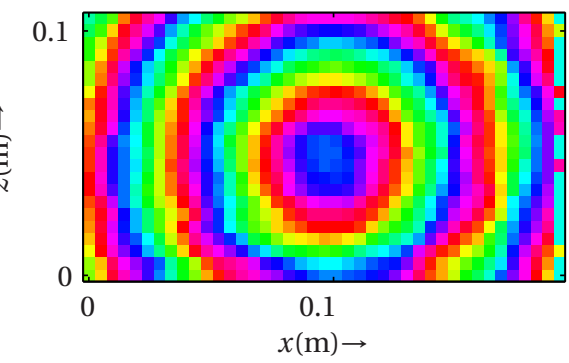

(j) cross spectrum (phase)

Figure 5.12 continued

The transfer function is calculated for each of these time-series and the result is interpolated to an equidistant grid of 41 points for each line. The total number of field points is 902: 41 grid points at 22 lines.

To compare the results, the magnitude and phase of the transfer function from reference sensor 1 to the field sensors are depicted. Figures 5.12(a) and 5.12(b) depict the results based on the multi-taper method (MTM) and total least squares (TLS) transfer estimation. It can be seen that the result is quite consistent with the theoretical response of a monopole source. In figures 5.12(c) and 5.12(d), the transfer function of the Welch method is depicted. In this case, each of the timeseries of 2048 samples is divided into 10 time series of 204 samples which have no overlap. Although the phase is accurate, the amplitude has an appearance which is more noisy than in the case of the Multi-taper method. Nevertheless, the result is considered to be practically useful. To paint a clearer picture of the differences between the Welch method and the multi-taper method, it is recommended to use a source which produces a sound field that varies more rapidly with frequency. Figure 5.12(e) and figure 5.12(f) depict the transfer function calculated using the conventional least squares method of equation 5.41. The second reference sensor has not been used in this calculation. A similarly accurate result has been 
achieved. A possible explanation is that the noise of the reference sensor is considerably smaller than the noise at the field sensor because the latter sensor moves on rails. The result can also be explained by the fact that noise is often far from white in practice such that subtle differences in the least squares problems under the assumption of white noise have little effect in practice.

Figures 5.13(g) and 5.13(h) depict the transfer function which has been calculated using a single taper. The taper is the DPSS where $2 W T=4$. It can be seen that the result contains many spikes. At these locations, noise causes the reference signals to be too low such that the ratio of the field signal and the reference signal is much too high. Hence, it can be concluded that it is necessary to perform solve a least squares problem over multiple tapers to achieve an accurate result. Figures 5.13(i) and 5.13(j) depict the cross spectrum. Its absolute value is highly distorted by source strength variation even though the number of tapers is large. This means that transfer estimation is necessary as argued in section 5.5.1.

The inverse method used is TRIM (see chapter 4). The source grid consists of $96 \times 58=5568$ Linear quadrilateral elements and the field data are interpolated to a grid of $41 \times 22=902$ points (see figure 5.13 ). Note that the number of source and field points is larger than they are in the other problems considered in this thesis. The regularization method used is TSVD and the SVD is calculated using the Lanczos SVD algorithm (see chapter 3).

The L-curve is depicted in figure 5.14(a) and figures 5.14(b) through 5.14(d) display an oversmoothed solution, an appropriately smoothed solution and an undersmoothed solution respectively. The solutions represent the transfer from the reference sensor to the normal velocity at the source, which is equal to the normal velocity at the source up to a constant. Since the different ways to estimate this constant have not been investigated in this study, the results are given aside from an arbitrary constant. The exact solution is a circular area of high velocity with a diameter of $20 \mathrm{~mm}$ and a velocity of zero elsewhere. The inverse solutions give confidence that the current method is competitive with the case where the sensors are

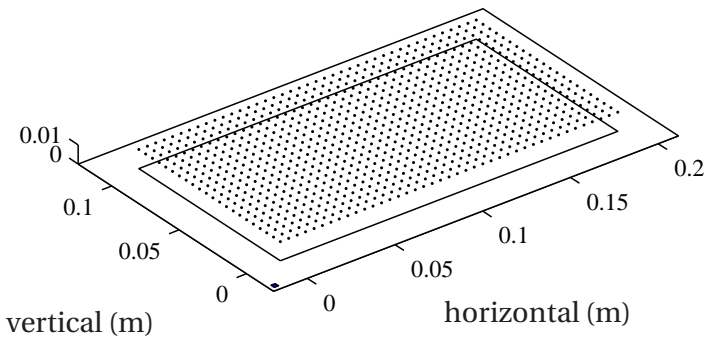

Figure 5.13: Geometry of the acoustic model. An element is displayed at the bottom left. 


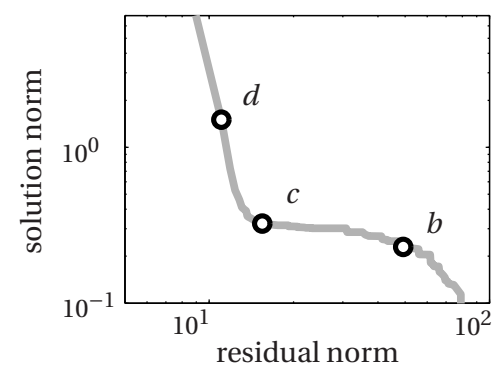

(a) L-curve

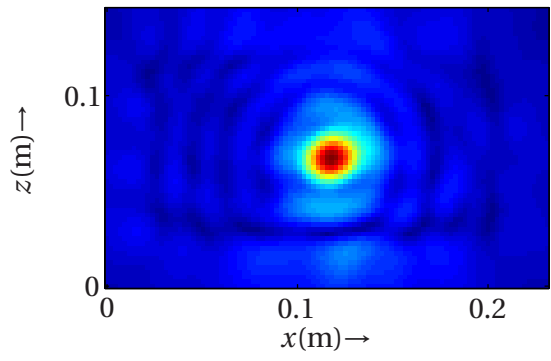

(c) solution corresponding to point $c$

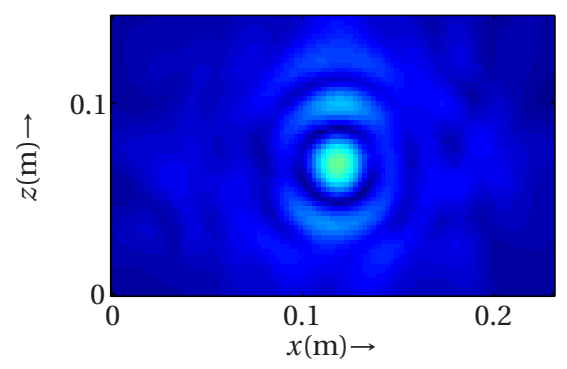

(b) solution corresponding to point $b$

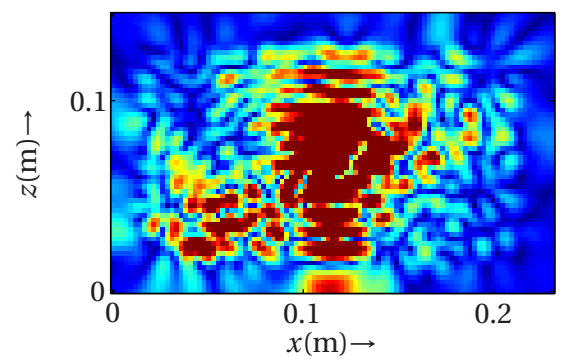

(d) solution corresponding to point $d$

Figure 5.14: Inverse solution using measurement data corresponding to figure 5.12(a). (Transfer function from the reference sensor to the source, absolute value)

standing still.

\subsection{Summary}

This chapter has considered statistical signal processing techniques for moving sensors. The theory of Moving Frame Nearfield Acoustic Holography (MFNAH) has been used to study the Doppler effect in the case where a single sensor is used to gather both spatial information and temporal information. It has been shown that the frequency shift due to the Doppler effect is smaller than the smallest bandwidth which can be resolved in signal processing, independent of the speed of the sensor.

Some aspects of statistical signal processing, such as the cross-spectral matrix and principal component analysis (PCA), have been introduced to form the theoretical basis of the next chapters.

The multi-taper method has been presented as a signal processing technique which minimizes leakage and total least squares has been shown to be the transfer estimator which is optimally robust to noise. It can be concluded from an experimental case study that estimating the transfer function contrary to the cross spec- 
trum has a large impact on the calculated sound field. Although the Welch method has yielded practically useful results, the results of the Multi-taper method have been slightly more accurate and further research is necessary to quantify the exact differences between these methods in the current application. Transfer estimation based on total least squares has yielded similar results as transfer estimation based on conventional least squares. 


\section{Chapter 6}

\section{Inverse acoustics using cross-spectral matrices}

\subsection{Introduction}

This chapter considers inverse problems which involve cross-spectral matrices. These problems are practically useful for two reasons. Firstly, the theoretical basis of regularization deals with physical quantities such as pressure and velocity but in practice, the sound field is usually known in the form of cross spectra. Secondly, it is often practically useful to calculate cross-spectral matrices of the source. An important example is the sound intensity, which is half the cross spectrum between pressure and velocity.

Conventional inverse acoustic techniques do not provide a direct solution to the cross-spectral problem. In this chapter, the problem is reformulated to be a linear inverse problem such that the singular value decomposition (SVD) is available for this problem. Conventional regularization techniques can be applied to this problem, leading to results which are new in nearfield source localization.

The main scientific contribution of this chapter is the fact that it is shown that the SVD has a specific structure, which makes it possible to solve the inverse problem in a time which is similar to the existing methods. This structure also makes it possible to write the existing inverse acoustic techniques for the cross-spectral problem in terms of this SVD, which leads to a deeper understanding of the existing techniques as well as the new approach.

This chapter is built up as follows. In section 6.2, the problem is defined concisely, existing techniques are reviewed and a new technique is proposed. Section 6.3 compares the effects of regularization in the existing and new techniques. A case study is presented in section 6.4. Further theoretical considerations and 
applications are considered in sections $6.5,6.7$ and 6.6. A summary is given in section 6.8 .

\subsection{Cross-spectral modeling}

\subsubsection{Sound intensity}

The sound intensity is defined to be the acoustic energy flux per unit of area. For deterministic signals in the frequency domain, the sound intensity is (see also equation 2.8)

$$
\vec{I}_{A}=\frac{1}{2} \operatorname{Re}\left(p \vec{v}^{*}\right)
$$

The expected value of the intensity is relevant for the random signals $\hat{P}(t)$ and $\hat{V}(t)$ at some location $\vec{x}$. This leads to the following expression in the frequency domain.

$$
\vec{I}_{A}=\frac{1}{2} \operatorname{Re}\left(\mathbf{S}_{p v}\right)
$$

If several locations $\vec{x}_{i}, i \in\{1,2, \ldots, n\}$ are considered, the cross spectrum between the pressure and velocity at these locations is a matrix. Only the diagonal terms are relevant to the sound intensity because the cross spectrum between the pressure at one point and the velocity at another has no impact on the expected value of the acoustic energy flux.

\subsubsection{Problem formulation}

As noted in section 5.3.2, four variables are necessary to describe the transfer from one cross spectrum to another in general. The source vibration is represented by the vector-valued signals $\hat{S}(t)$ and $\hat{T}(t)$. They can represent the pressure and velocity at the source for example. The field is represented by the signals $\hat{F}(t)$ and $\hat{G}(t)$. Each of the scalar valued signals $\hat{F}_{1}(t), \hat{F}_{2}(t), \cdots, \hat{F}_{\hat{m}}(t)$ or $\hat{G}_{1}(t), \hat{G}_{2}(t), \cdots, \hat{G}_{m}(t)$ can be the pressure and velocity at a field point for example.

The problem of calculating cross spectrum matrices is a problem in the form of a Sylvester equation. It has been presented in equation 5.23 and it is repeated here.

$$
\mathbf{S}_{\mathbf{f g}}=\mathbf{H}_{\mathrm{fs}} \mathbf{S}_{\mathbf{s t}} \mathbf{H}_{\mathrm{gt}}^{H} \quad \text { where } \quad\left\{\begin{array}{l}
\mathbf{H}_{\mathrm{fs}} \mathbf{s}=\mathbf{f} \\
\mathbf{H}_{\mathrm{gt}} \mathbf{t}=\mathbf{g}
\end{array}\right.
$$

The sound intensity depends on the diagonal elements of the cross-spectral matrix between pressure and velocity at the source but the off-diagonal terms are also required to formulate the acoustic problem in terms of cross spectra. The 
off-diagonal elements contain the correlations between pressure at point $i$ and velocity at point $j$. Although these correlations do not influence the radiated sound power, they do influence both the auto-spectral and cross-spectral coefficients of the sound field. For example, if $i$ and $j$ are two correlated point sources, their sound fields exhibit an interference pattern but if the sources are uncorrelated, this interference pattern is absent. Therefore, it is impossible to develop a model that describes the transfer from sound intensity at the source to sound intensity in the field without making assumptions about the correlation between the sources. Such assumptions are not made in this chapter. Instead, the model describes the transfer between two cross spectrum matrices. Hence, the inverse techniques calculate the cross-spectral matrix between pressure and velocity - not merely the sound intensity vector.

\subsubsection{Review of existing inverse techniques}

The inverse techniques which are considered in this chapter solve equation 6.3 for $\mathbf{S}_{\mathbf{s t}}$. If the transfer matrices are square and well conditioned, the exact source cross spectrum can be calculated by multiplying equation 6.3 at the left and right-hand

sides with the inverse of the $\mathbf{H}_{\mathbf{f s}}$ and $\mathbf{H}_{\mathbf{g t}}^{H}$ respectively. In the case where the transfer matrices are rectangular and ill-conditioned, it is intuitive to multiply both sides with a regularized inverse.

The existing regularization techniques are based on this idea. They are best explained from their physical background. The explanation is simplified by assuming that $\mathbf{f}(\omega)$ and $\mathbf{g}(\omega)$ are the same: either the measured pressure or acoustic particle velocity. The derivation of these techniques is started by separating the measured cross-spectral matrix into a superposition of $k$ uncorrelated sound fields. This is the essence of spatial transformation of sound fields (STSF), which is widely used in acoustical signal processing [22].

$$
\mathbf{S}_{\mathbf{f f}}=\sum_{i=1}^{k} \mathbf{f}_{i} \mathbf{f}_{i}^{H}
$$

Each of the vectors $\mathbf{f}_{i} \in \mathbb{C}^{\hat{m}}$ can be interpreted as a measured pressure or velocity in the frequency domain. The vectors are correlated with themselves and uncorrelated with the other vectors. There are many ways to decompose a random sound field into incoherent components but the decomposition does not influence the solution if it has the form of equation 6.4. A natural choice is PCA (see section 5.3.3).

To understand the differences between the methods considered in this section, it is important to distinguish between operations which require regularization and operations which do not. To make this distinction clear in a brief manner, the term 
to reconstruct is used to describe a regularized inverse operation. The term to calculate is used to describe a forward calculation which does not require regularization.

There are several ways to determine the velocity $\mathbf{s}$ and the pressure $\mathbf{t}$ at the source. In the first method, the velocity is reconstructed and the pressure is calculated from this reconstructed velocity field using the impedance matrix $\mathbf{H}_{\mathbf{t s}}$

$$
\begin{aligned}
& \mathbf{s}_{i}^{(1)}=\mathbf{H}_{\mathbf{f s}}^{\dagger \alpha} \mathbf{f}_{i} \quad ; \quad i=1,2, \ldots, k \\
& \mathbf{t}_{i}^{(1)}=\mathbf{H}_{\mathbf{t s}} \mathbf{s}_{i}^{(1)} ; i=1,2, \ldots, k
\end{aligned}
$$

Where (.) ${ }^{\dagger \alpha}$ denotes the regularized inverse matrix (see also section 3.2) and $\alpha$ is the regularization parameter. The matrix $\mathbf{H}_{\mathbf{t s}} \in \mathbb{C}^{n \times \hat{n}}$ is the impedance matrix: the transfer matrix from the velocity to the pressure at the source. The cross-spectral matrix at the source can be found by adding the contributions of the sound fields

$$
\mathbf{S}_{\mathbf{s t}}^{(1)}=\sum_{i=1}^{n_{i}} \mathbf{s}_{i}^{(1)} \mathbf{t}_{i}^{(1) H}
$$

The approach of reconstructing the velocity and calculating the pressure from it has been used in articles by Williams and others (see [10,98]). By substitution, it can be seen that equations 6.4 through 6.7 can be summarized as follows

$$
\mathbf{S}_{\mathbf{s t}}^{(1)}=\mathbf{H}_{\mathbf{f s}}^{\dagger \alpha} \mathbf{S}_{\mathbf{f f}} \mathbf{H}_{\mathbf{f s}}^{\dagger \alpha H} \mathbf{H}_{\mathbf{t s}}^{H}
$$

Equation 6.8 gives the exact solution the matrices are square and well-conditioned, but regularization errors can possibly have an unnecessarily large impact on the result. Any regularization errors in the velocity are translated directly to errors in the pressure, even if the pressure components are well-conditioned. A clear alternative is to reconstruct the pressure and to calculate the velocity. This approach requires some care because the calculation of velocity from the pressure is a mildly ill-posed problem.

$$
\begin{aligned}
\mathbf{t}_{i}^{(2)} & =\mathbf{H}_{\mathbf{f t}}^{\dagger \alpha} \mathbf{f}_{i} \quad ; \quad i=1,2, \ldots, k \\
\mathbf{s}_{i}^{(2)} & =\mathbf{H}_{\mathbf{t s}}^{-1} \mathbf{t}_{i}^{(2)} ; i=1,2, \ldots, k \\
\mathbf{S}_{\mathbf{s t}}^{(2)} & =\sum_{i=1}^{k} \mathbf{s}_{i}^{(2)} \mathbf{t}_{i}^{(2) H} \quad \text { hence } \\
\mathbf{S}_{\mathbf{s t}}^{(2)} & =\mathbf{H}_{\mathbf{t s}}^{-1} \mathbf{H}_{\mathbf{f t}}^{\dagger \alpha} \mathbf{S}_{\mathbf{f f}} \mathbf{H}_{\mathbf{f t}}^{\dagger \alpha H}
\end{aligned}
$$

A third alternative is to calculate the pressure and velocity separately, using a regularized inverse for both quantities. This means that the problem has two regular- 


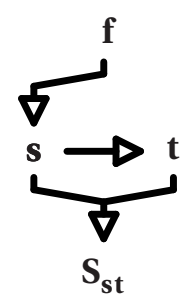

(a) Eq. 6.8

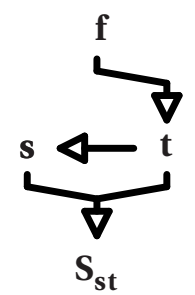

(b) Eq. 6.12

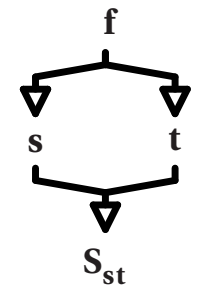

(c) Eq. 6.16

Figure 6.1: Schematic representation of the order in which the source pressure $\mathbf{s}$, the source velocity $\mathbf{t}$ and the cross-spectral matrix $\mathbf{S}_{\mathbf{s t}}$ are calculated using the three existing inverse techniques.

ization parameters.

$$
\begin{aligned}
\mathbf{t}_{i}^{(3)} & =\mathbf{H}_{\mathbf{f t}}^{\dagger \alpha} \mathbf{f}_{i} \quad ; \quad i=1,2, \ldots, k \\
\mathbf{s}_{i}^{(3)} & =\mathbf{H}_{\mathbf{f s}}^{\dagger \beta} \mathbf{f}_{i} ; i=1,2, \ldots, k \\
\mathbf{S}_{\mathbf{s t}}^{(3)} & =\sum_{i=1}^{k} \mathbf{s}_{i}^{(3)} \mathbf{t}_{i}^{(3) H} \quad \text { hence } \\
\mathbf{S}_{\mathbf{s t}}^{(3)} & =\mathbf{H}_{\mathbf{f t}}^{\dagger \alpha} \mathbf{S}_{\mathbf{f f}} \mathbf{H}_{\mathbf{f s}}^{\dagger \beta H}
\end{aligned}
$$

Here, $\beta$ is the second regularization parameter. Although methods (2) and (3) have not been found in literature, they are referred to as existing techniques nonetheless, because they are considered to be trivial extensions to method (1). The three methods that have been derived in this section are depicted schematically in figure 6.1. For completeness, it is mentioned that each of the three techniques simplify to the same equation in case the spectral matrix pressure-pressure or velocity-velocity is reconstructed. This equation has been proposed by Nelson and Yoon [48, 104]. Their article makes use of cross-spectral matrices and in the current notation, their solution is as follows.

$$
\mathbf{S}_{\mathbf{s s}}^{(4)} \approx \mathbf{H}_{\mathbf{f s}}^{\dagger \alpha} \mathbf{S}_{\mathbf{f f}} \mathbf{H}_{\mathbf{f s}}^{\dagger \alpha H}
$$

In summary, this section has discussed the existing inverse techniques for cross spectra. All methods are the same if regularization is not applied but the regularization errors are different. Contrary to the new method which is proposed in the next section, the existing methods use the inverse operations for the pressure or velocity vectors, rather than cross-spectral matrices. 


\subsubsection{Reformulation of the equation to a matrix-vector equation}

In this section, the matrix-matrix equation 6.3 is reformulated in the form of a matrix-vector equation, which makes it possible to apply standard regularization techniques to the cross-spectral problem. This approach also provides a solid theoretical basis to compare the existing techniques and the ones that follow directly from this matrix-vector equation.

In the context of aeroacoustic source localization, a similar approach has been published by Brooks [9] for the case where $\mathbf{s}=\mathbf{t}$ and $\mathbf{f}=\mathbf{g}$. Since the transfer matrix is full and it can easily contain a million by a million elements, the computational effort is so large that the problem cannot be solved on any computer. To make it possible to solve the equations, Brooks assumes that the correlations between sources which are some distance apart are zero. Although exact computation times have not been published, it is known that a large mainframe computer is necessary to perform the calculations even after this approximation. The next section extends the work of Brooks by showing that the SVD has a specific structure, which makes it possible to solve the SVD within a minute using a personal computer and to study the relation between the new and the existing methods. The identification of the structured SVD in the context of inverse acoustics is the main scientific contribution of this chapter. Since this structure does not occur if the correlation between some sources is assumed to be zero, the results of the method which is presented here are different from the results obtained by Brooks. The method discussed here is therefore referred to as the new method.

Equation 6.3 shows that there exists a linear relation between each element of $\mathbf{S}_{\mathbf{f g}} \in \mathbb{C}^{\hat{m} \times m}$ and the matrix $\mathbf{S}_{\mathbf{s t}} \in \mathbb{C}^{\hat{n} \times n}$. It can therefore be written as a matrix-vector equation. To arrive at that result, equation 6.3 is first written as an element-wise summation:

$$
\left(\mathbf{S}_{\mathbf{f g}}\right)_{i j}=\sum_{k} \sum_{l}\left(\mathbf{H}_{\mathbf{f s}}\right)_{i k} \overline{\left(\mathbf{H}_{\mathbf{g t}}\right)_{j l}}\left(\mathbf{S}_{\mathbf{s t}}\right)_{k l}
$$

The four-dimensional operator $\left(\mathbf{H}_{\mathbf{f s}}\right)_{i k} \overline{\left(\mathbf{H}_{\mathbf{g t}}\right)_{j l}}$ can be written as a matrix with a specific block structure. This matrix, referred to as the Kronecker product of $\overline{\mathbf{H}}_{\mathbf{g t}} \in$ $\mathbb{C}^{m \times n}$ and $\mathbf{H}_{\mathbf{f s}} \in \mathbb{C}^{\hat{m} \times \hat{n}}$ is defined as [29]:

$$
\begin{aligned}
& \overline{\mathbf{H}}_{\mathrm{gt}} \otimes \mathbf{H}_{\mathrm{fs}}= \\
& {\left[\begin{array}{cccc}
\left(\overline{\mathbf{H}}_{\mathbf{g t}}\right)_{11} \cdot \mathbf{H}_{\mathbf{f s}} & \left(\overline{\mathbf{H}}_{\mathbf{g t}}\right)_{12} \cdot \mathbf{H}_{\mathbf{f s}} & \ldots & \left(\overline{\mathbf{H}}_{\mathbf{g t}}\right)_{1 n} \cdot \mathbf{H}_{\mathbf{f s}} \\
\left(\overline{\mathbf{H}}_{\mathbf{g t}}\right)_{21} \cdot \mathbf{H}_{\mathbf{f s}} & \left(\overline{\mathbf{H}}_{\mathbf{g t}}\right)_{22} \cdot \mathbf{H}_{\mathbf{f s}} & \ldots & \left(\overline{\mathbf{H}}_{\mathbf{g t}}\right)_{2 n} \cdot \mathbf{H}_{\mathbf{f s}} \\
\vdots & \vdots & \ddots & \vdots \\
\left(\overline{\mathbf{H}}_{\mathbf{g t}}\right)_{m 1} \cdot \mathbf{H}_{\mathbf{f s}} & \left(\overline{\mathbf{H}}_{\mathbf{g t}}\right)_{m 2} \cdot \mathbf{H}_{\mathbf{f s}} & \ldots & \left(\overline{\mathbf{H}}_{\mathbf{g t}}\right)_{m n} \cdot \mathbf{H}_{\mathbf{f s}}
\end{array}\right] \in \mathbb{C}^{m \hat{m} \times n \hat{n}}}
\end{aligned}
$$


Along with the Kronecker product, the vec operator is introduced. It transforms a matrix to a large vector by 'stacking' the columns. Let $\mathbf{S}_{\mathbf{s t}} \in \mathbb{C}^{n \times \hat{n}}$ be a matrix with columns $\left(\mathbf{S}_{\mathbf{s t}}\right)_{i}$, then

$$
\operatorname{vec}\left(\mathbf{S}_{\mathbf{s t}}\right)=\left\{\begin{array}{c}
\left(\mathbf{S}_{\mathbf{s t}}\right)_{1} \\
\left(\mathbf{S}_{\mathbf{s t}}\right)_{2} \\
\vdots \\
\left(\mathbf{S}_{\mathbf{s t}}\right)_{n}
\end{array}\right\} \in \mathbb{C}^{n \cdot \hat{n} \times 1} \quad \text { with } \quad\left(\mathbf{S}_{\mathbf{s t}}\right)_{i}=\left\{\begin{array}{c}
\left(\mathbf{S}_{\mathbf{s t}}\right)_{1 i} \\
\left(\mathbf{S}_{\mathbf{s t}}\right)_{2 i} \\
\vdots \\
\left(\mathbf{S}_{\mathbf{s t}}\right)_{n i}
\end{array}\right\}
$$

The linear relation in equation 6.18 is written as a matrix-vector product using these operations.

$$
\left(\overline{\mathbf{H}}_{\mathbf{g t}} \otimes \mathbf{H}_{\mathbf{f s}}\right) \operatorname{vec}\left(\mathbf{S}_{\mathbf{s t}}\right)=\operatorname{vec}\left(\mathbf{S}_{\mathbf{f g}}\right)
$$

This matrix-vector equation can be solved simply by taking a regularized inverse as defined in equation 3.10 .

$$
\left(\overline{\mathbf{H}}_{\mathbf{g t}} \otimes \mathbf{H}_{\mathbf{f s}}\right)^{\dagger \alpha} \operatorname{vec}\left(\mathbf{S}_{\mathbf{f g}}\right)=\operatorname{vec}\left(\mathbf{S}_{\mathbf{s t}}\right)
$$

In the rest of this chapter, the existing methods of section 6.2 .3 are compared to the new method.

\subsection{Regularization}

\subsubsection{The SVD of the Kronecker product of two matrices}

The matrix that follows from the Kronecker product tends to be too large to be stored in the memory of any computer, because its size is $m \cdot \hat{m} \times n \cdot \hat{n}$. However, only the SVDs of the two transfer matrices are stored in practice and the SVD of their Kronecker product is calculated from them. This is achieved as follows. Let the SVD of $\mathbf{H}_{\mathbf{f s}}$ and $\mathbf{H}_{\mathbf{g t}}$ be given by:

$$
\begin{aligned}
& \mathbf{H}_{\mathbf{f s}}=\mathbf{U}_{\mathbf{f s}} \Sigma_{\mathbf{f s}} \mathbf{V}_{\mathbf{f s}}^{H}=\sum_{j} \mathbf{u}_{\mathbf{f s}} \sigma_{\mathbf{f s}_{j}} \mathbf{v}_{\mathbf{f s}}^{H} \\
& \mathbf{H}_{\mathbf{g t}}=\mathbf{U}_{\mathbf{g t}} \boldsymbol{\Sigma}_{\mathbf{g t}} \mathbf{V}_{\mathbf{g t}}^{H}=\sum_{i} \mathbf{u}_{\mathbf{g t}_{i}} \sigma_{\mathbf{g t}_{i}} \mathbf{v}_{\mathbf{g t}_{i}}^{H}
\end{aligned}
$$

Now, the $\mathbf{U}$ matrix of a Kronecker product is equal to the Kronecker product of the two $\mathbf{U}$ matrices involved [29]. The same holds for the $\boldsymbol{\Sigma}$ and $\mathbf{V}$ matrices.

$$
\begin{aligned}
& \left(\overline{\mathbf{H}}_{\mathbf{g t}} \otimes \mathbf{H}_{\mathbf{f s}}\right)=\mathbf{U} \boldsymbol{\Sigma} \mathbf{V}^{H} \\
& \quad=\left(\overline{\mathbf{U}}_{\mathbf{g t}} \otimes \mathbf{U}_{\mathbf{f s}}\right)\left(\boldsymbol{\Sigma}_{\mathbf{g t}} \otimes \boldsymbol{\Sigma}_{\mathbf{f s}}\right)\left(\overline{\mathbf{V}}_{\mathbf{g t}} \otimes \mathbf{V}_{\mathbf{f s}}\right)^{H} \\
& \quad=\sum_{i} \sum_{j}\left(\overline{\mathbf{u}}_{\mathbf{g t}_{i}} \otimes \mathbf{u}_{\mathbf{f s}}\right)\left(\sigma_{\mathbf{g t}_{i}} \cdot \sigma_{\mathbf{f s}_{j}}\right)\left(\overline{\mathbf{v}}_{\mathbf{g t}} \otimes \mathbf{v}_{\mathbf{f s}}\right)^{H}
\end{aligned}
$$


Note that the product $\sigma_{\mathbf{g t}_{i}} \cdot \sigma_{\mathbf{f s}_{j}}$ is a singular value of the large matrix for each $i$ and each $j$.

There exists a different approach to derive an SVD for the cross-spectral problem. The higher order SVD (HOSVD) discussed in [43] can be applied to the fourdimensional operator in equation 6.18 . This approach yields the same singular values and vectors as equation 6.25, such that the reconstructed source of this method is also the same.

\subsubsection{Filter functions for cross spectra}

In this section, the regularized inverse matrices of the new and existing methods are compared. Consider existing method (3), where the sound intensity is found by reconstructing pressure and velocity separately and multiplying the result (see equation 6.16). This equation is written as a matrix-vector equation using the Kronecker product

$$
\left.\overline{\left(\mathbf{H}_{\mathbf{g t}}^{\dagger \beta}\right.} \otimes \mathbf{H}_{\mathbf{f s}}^{\dagger \alpha}\right) \operatorname{vec}\left(\mathbf{S}_{\mathbf{f g}}\right)=\operatorname{vec}\left(\mathbf{S}_{\mathbf{s t}}\right)
$$

In terms of the SVDs of the two matrices, the regularized inverse matrix is

$$
\left.\overline{\left(\mathbf{H}_{\mathbf{g t}}^{\dagger \beta}\right.} \otimes \mathbf{H}_{\mathbf{f s}}^{\dagger \alpha}\right)=\sum_{i} \sum_{j}\left(\overline{\mathbf{v}}_{\mathbf{g t}_{i}} \otimes \mathbf{v}_{\mathbf{f} \mathbf{s}_{j}}\right)\left(\frac{\mathscr{F}_{\beta}\left(\sigma_{\mathbf{g t}_{i}}\right) \cdot \mathscr{F}_{\alpha}\left(\sigma_{\mathbf{f s}_{j}}\right)}{\sigma_{\mathbf{g t}_{i}} \cdot \sigma_{\mathbf{f s}_{j}}}\right)\left(\overline{\mathbf{u}}_{\mathbf{g t}_{i}} \otimes \mathbf{u}_{\mathbf{f s}}\right)^{H}
$$

where $\mathscr{F}_{\alpha}(\cdot)$ and $\mathscr{F}_{\beta}(\cdot)$ are the applied filter function with regularization parameter $\alpha$ and $\beta$ respectively. The regularized inverse matrix contains the product of the two separate filter functions.

The regularization method (1), where velocity is reconstructed and pressure is calculated from velocity (see equation 6.8), cannot be expressed in this form because it is not a filter applied to the singular values of the matrix $\overline{\mathbf{H}}_{\mathbf{g t}} \otimes \mathbf{H}_{\text {fs }}$ in general. In the specific case that the cross spectrum velocity - velocity is reconstructed based on the cross spectrum pressure - pressure, method (1) simplifies to method (4) (see equation 6.17). This equation can be expressed as follows

$$
\left(\overline{\mathbf{H}_{\mathbf{f s}}^{\dagger \alpha}} \otimes \mathbf{H}_{\mathbf{f s}}^{\dagger \alpha}\right)=\sum_{i} \sum_{j}\left(\overline{\mathbf{v}}_{\mathbf{f} \mathbf{s}_{i}} \otimes \mathbf{v}_{\mathbf{f s}}\right)\left(\frac{\mathscr{F}_{\alpha}\left(\sigma_{\mathbf{f s}_{i}}\right) \cdot \mathscr{F}_{\alpha}\left(\sigma_{\mathbf{f s}_{j}}\right)}{\sigma_{\mathbf{f s}_{i}} \cdot \sigma_{\mathbf{f s}_{j}}}\right)\left(\overline{\mathbf{u}}_{\mathbf{f} \mathbf{s}_{i}} \otimes \mathbf{u}_{\mathbf{f s}}\right)^{H}
$$

Finally, the new method (equation 6.22) is considered. From equation 6.25 it is seen that the regularized inverse is

$$
\begin{aligned}
\left(\overline{\mathbf{H}}_{\mathbf{g t}} \otimes \mathbf{H}_{\mathbf{f s}}\right)^{\dagger \alpha} & =\mathbf{V} \boldsymbol{\Sigma}^{\dagger \alpha} \mathbf{U}^{H} \\
& =\sum_{i} \sum_{j}\left(\overline{\mathbf{v}}_{\mathbf{g t}_{i}} \otimes \mathbf{v}_{\mathbf{f s}}\right)\left(\frac{\mathscr{F}_{\alpha}\left(\sigma_{\mathbf{g t}_{i}} \cdot \sigma_{\mathbf{f s}_{j}}\right)}{\sigma_{\mathbf{g t}_{i}} \cdot \sigma_{\mathbf{f s}_{j}}}\right)\left(\overline{\mathbf{u}}_{\mathbf{g t}_{i}} \otimes \mathbf{u}_{\mathbf{f} \mathbf{f}_{j}}\right)^{H}
\end{aligned}
$$


In this case, the filter function is applied to the product of the two singular values. In summary, the filter functions of method (3), method (4) and the new method are, in respective order

$$
\begin{aligned}
\mathscr{F}_{(3)}\left(\sigma_{\mathbf{f s}_{i}}, \sigma_{\mathbf{g t}_{j}}\right) & =\mathscr{F}_{\alpha}\left(\sigma_{\mathbf{f s}_{i}}\right) \mathscr{F}_{\beta}\left(\sigma_{\mathbf{g t}_{j}}\right) \\
\mathscr{F}_{(4)}\left(\sigma_{\mathbf{f s}_{i}}, \sigma_{\mathbf{f s}_{j}}\right) & =\mathscr{F}_{\alpha}\left(\sigma_{\mathbf{f s}_{i}}\right) \mathscr{F}_{\alpha}\left(\sigma_{\mathbf{f s}_{j}}\right) \\
\mathscr{F}_{(N e w)}\left(\sigma_{\mathbf{g t}_{i}}, \sigma_{\mathbf{f s}_{j}}\right) & =\mathscr{F}_{\alpha}\left(\sigma_{\mathbf{f s}_{j}} \sigma_{\mathbf{g t}_{i}}\right)
\end{aligned}
$$

Since these filters are real functions of two variables, they can be displayed in a contour plot. Figure 6.2 depicts $\mathscr{F}_{(3)}$ and $\mathscr{F}_{(N e w)}$ for the case study performed in section 6.4. The vertical axis is used for the singular value number $i$, which corresponds to the transfer from velocity at the source to pressure at the field and the horizontal axis is used for the singular value number $j$, which corresponds to the transfer from pressure at the source to pressure at the field. It can be seen that the filters are quite different. The existing filters have a rectangular shape: pressure and velocity are reconstructed separately and the result is multiplied (see figures 6.2(a) and 6.2(b)). Hence, component $(i, j)$ of the cross-spectral matrix is filtered out if either of the singular values are small. On the other hand, the new filters also allow component $(i, j)$ to pass if $i$ is high and $j$ is low and vice versa (see figures 6.2(c) and $6.2(\mathrm{~d}))$, but the component $(j, j)$ is filtered out.

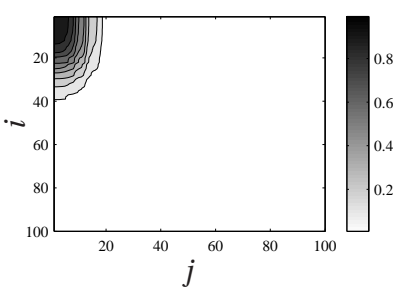

(a) $\mathscr{F}_{(3)}$ (Tikhonov)

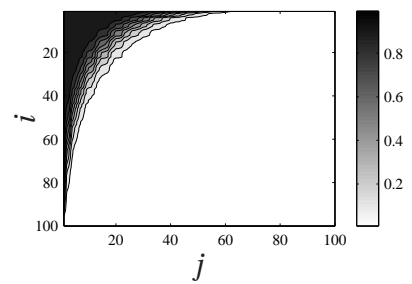

(c) $\mathscr{F}_{(\text {New })}$ (Tikhonov)

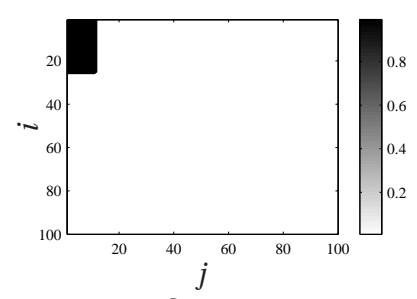

(b) $\mathscr{F}_{(3)}(\mathrm{TSVD})$

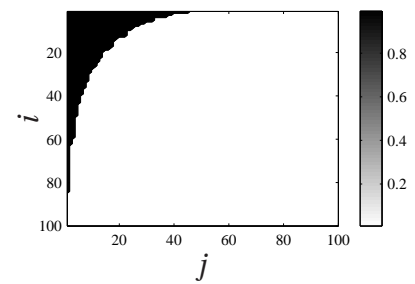

(d) $\mathscr{F}($ New) $($ TSVD)

Figure 6.2: Contour plot of the filter functions in accordance with equations 6.16 and 6.22 (Normal velocity, $500 \mathrm{~Hz}$ ) 
On one hand, this property of the new method is an advantage. It means that the knowledge of component $(i, j)$ is used to reconstruct the source more accurately whereas the existing methods do not make use of this knowledge. On the other hand, this property can lead to counterintuitive results. If the source matrix is the spectral matrix velocity-velocity or pressure-pressure, then the exact source is positive semidefinite. However, the reconstructed spectral matrix of the source is not positive semidefinite, because diagonal element $(j, j)$ is zero although component $(i, j)$ is nonzero. It is shown in section 6.7 that the source matrix which is reconstructed using the new method also tends to have a higher rank than the exact source matrix and that this is not the case for the existing methods.

\subsection{Case study}

\subsubsection{Introduction}

A case study is performed to compare the existing and new methods for cross spectra. First, a simulation study is performed to arrive at quantitative error results. Second, the methods are applied to measurement data and compared to laservibrometer results.

\subsubsection{Simulation study}

\section{Problem description}

The hard disk drive which is used throughout this thesis is used in both the simulation study and the experimental study. A detailed description of the properties is given in section 3.4.

A frequency of $500 \mathrm{~Hz}$ is used and the source is discretized using bilinear quadrilateral elements of $5 \times 5 \mathrm{~mm}$ (see figure 6.3). Two uncorrelated shapes of vibration are defined at the source. One is smooth, similar to a mode shape of a plate and

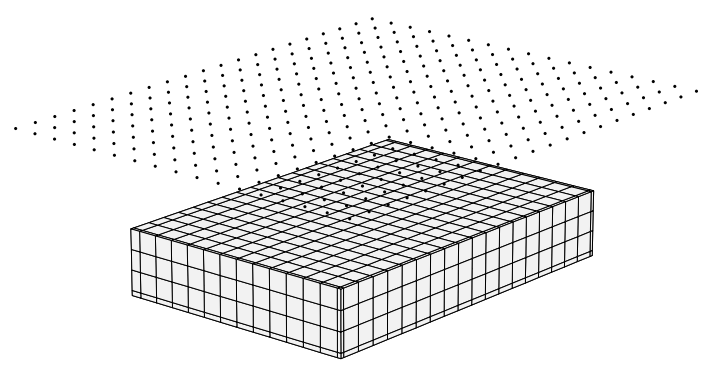

Figure 6.3: Geometry of the boundary element model with source elements and field points 
one is sharp and localized (see figures 6.4(b) and 6.4(c) respectively). The sharp peak makes the shape of vibration difficult to calculate. On the other hand, it is not far from noise sources that can be found in practice. The aim of this study is to approximate the sound intensity at each point at the source. Since this is a simulation study, the exact solution is known. It is depicted in figure 6.4(a)
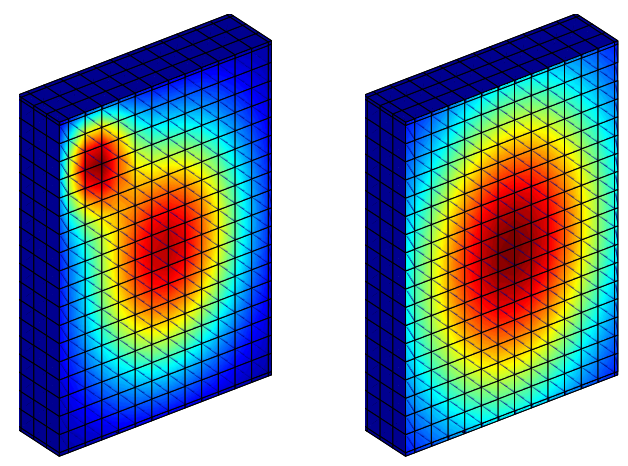

(a) Absolute value of the sound intensity $(500 \mathrm{~Hz})$ 


\section{Inverse methods}

Two existing methods are compared to the new method in this section. The first existing method is (1) (see equation 6.8), where velocity is reconstructed using conventional inverse acoustics and pressure is calculated from the reconstructed velocity using the impedance matrix. The second existing method is (3) (see equation 6.16), where both pressure and velocity are reconstructed using conventional inverse techniques. The new technique is based on the Kronecker product (equation 6.22).

The techniques require the choice of a regularization filter $\mathscr{F}_{\alpha}(\sigma)$ and a regularization parameter $\alpha$. The regularization filter is TSVD (see section 3.2). The optimal regularization parameter is selected based on the exact solution using the following procedure. The error between the reconstructed source and the exact solution is calculated for a range of 100 regularization parameters. The relative error $\gamma$ is calculated as the vector norm of the complex intensity at the nodes:

$$
\gamma=\frac{\sqrt{\sum_{i}\left|\left(\mathbf{S}_{\mathbf{s t}}\right)_{i i}-\left(\widetilde{\mathbf{S}}_{\mathbf{s t}}\right)_{i i}\right|^{2}}}{\sqrt{\sum_{i}\left|\left(\widetilde{\mathbf{S}}_{\mathbf{s t}}\right)_{i i}\right|^{2}}}
$$

Where $\mathbf{S}_{\mathbf{s t}}$ and $\widetilde{\mathbf{S}}_{\mathbf{s t}}$ are the reconstructed and exact cross spectrum matrices at the source respectively. The regularization parameter which has the smallest error norm is selected. Equation 6.16 involves two regularization parameters. Although it is possible to optimize both regularization parameters using the exact solution, a solution is chosen which is considered to be closer to engineering practice. Since a two-dimensional L-curve would be difficult to use, it is more practical to select one regularization parameter using the L-curve and to select the other parameter using knowledge of the selected regularization parameter, but not of the exact solution. The discrepancy principle is used to select the second regularization parameter. Pressure and velocity are both reconstructed using the same residual norm (see figure 6.5). A range of 100 residual norms is tried and the solution with the smallest error $\gamma$ (see equation 6.35) is selected. A number of other ways of calculating the second regularization parameter has been tested but this approach shows to be the most accurate result.

\section{Principal Component Analysis}

The simulated measurements are preprocessed in the same way experimental data is usually treated. Principal Component Analysis (PCA) (see section 5.3.3) is applied to remove some noise from the field spectrum by removing sources that are weak 

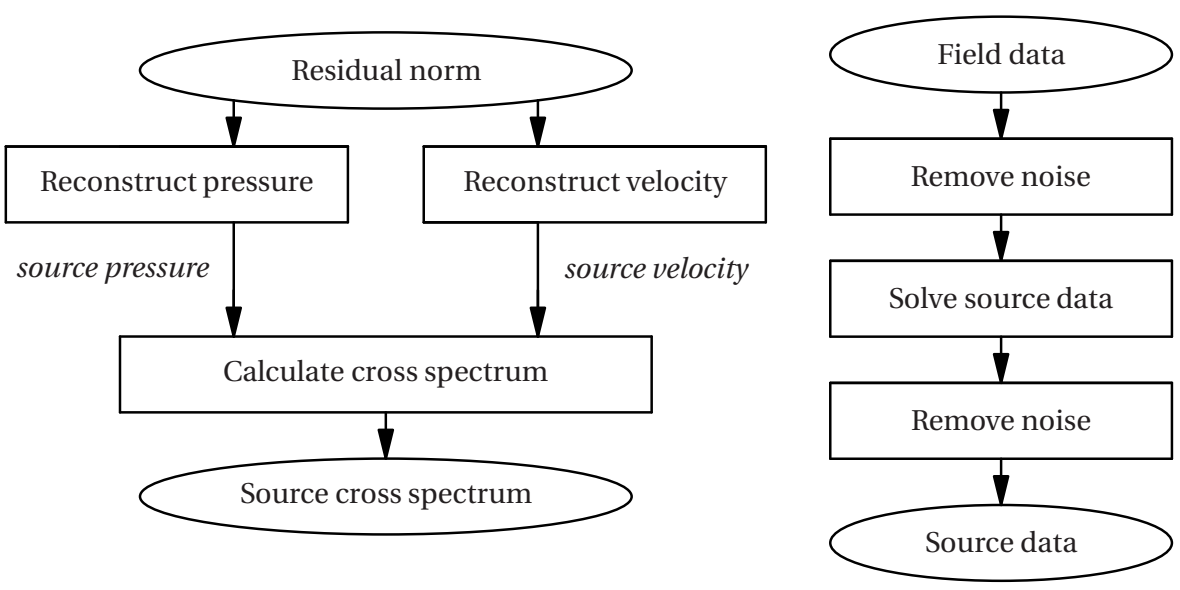

Figure 6.5: Matching of regularization parameters using the discrepancy principle

Figure 6.6: PCA can be applied to the source and the field data to remove noise

and uncorrelated to the dominant sources. The fact that two uncorrelated sources exist is assumed to be known.

Since the source matrix is the cross spectrum between pressure and velocity, and not for example the spectrum pressure-pressure, the derivation of chapter 5 does not apply directly. In this chapter, PCA means calculating the SVD and setting all but the first two singular values to zero. For a cross-spectral matrix $\mathbf{S}_{\mathbf{f g}}$ :

$$
\begin{aligned}
\mathbf{S}_{\mathbf{f g}} & =\mathbf{U}_{\mathbf{f g}} \Sigma_{\mathbf{f g}} \mathbf{V}_{\mathbf{f g}}{ }^{H} \\
\mathbf{S}_{\mathbf{f g}}^{\mathrm{PCA}} & =\mathbf{u}_{\mathbf{f g} 1} \cdot \sigma_{\mathbf{f g} 1} \cdot \mathbf{v}_{\mathbf{f g} 1}^{H}+\mathbf{u}_{\mathbf{f g} 2} \cdot \sigma_{\mathbf{f g} 2} \cdot \mathbf{v}_{\mathbf{f g} 2}^{H}
\end{aligned}
$$

As displayed in figure 6.6, PCA can be applied to the source and to the field cross spectrum. Since it is uncertain if PCA always improves the solution, all possible cases are compared: PCA is applied to the source, to the field, to both and to neither.

\section{Results}

The results of the simulation study are summarized in figure 6.7. The new technique is considerably more accurate than the existing techniques if PCA is not applied but the results are similar if PCA is applied to the field or to both. This can be explained by noting that the existing methods reconstruct the same number of uncorrelated sources which are present in the field data. The new method reconstructs more uncorrelated sources such that the information about the number of 
uncorrelated sources is not used (see also section 6.7). Since the existing methods do use this information, they do not require PCA at the source if PCA has been applied to the field, because the number of reconstructed uncorrelated sources is already 2. Consequently, it can be seen that the error in the cases 'Field' and 'Both' is equal for the existing methods.

The most accurate result has been obtained for an existing method, method (3), and a similar result is achieved by the new method. Method (1) provides the worst results in all cases. Possibly, this is caused by the calculated pressure being inaccurate such that the intensity is inaccurate as well. However, it is surprising to see that all of the reconstructions at the right half of figures 6.8 through 6.10, (figures 6.8(c), 6.8(d), 6.9(c), 6.9(d), 6.10(c) and 6.10(d)) are almost indistinguishable. In all of these cases, PCA has been applied to the field. This is the main contributing factor to the accuracy of these results.

The noise model does not include any bias errors or modeling inaccuracies. The high accuracy of the results illustrates the importance of such errors. Since the number of source nodes is larger than the number of sensors, the inverse problem is underdetermined, leading to an error in the absence of noise. This error is $6 \%$ for the current shape of vibration if numerical instabilities are neglected. The smallest error in the presence of noise is $10.5 \%$.

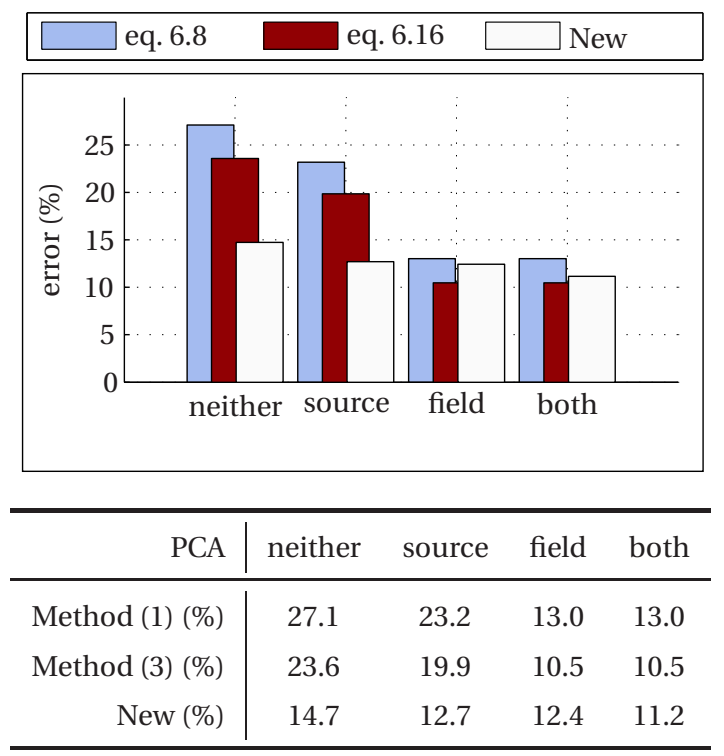

Figure 6.7: The relative errors in the simulation study, calculated using equation 6.35. The four cases consist of PCA being applied to the source, to the field, to both and to neither. 


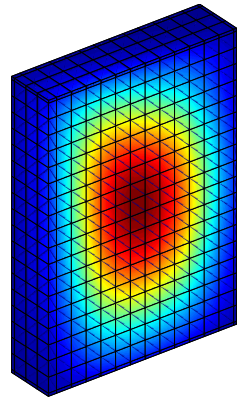

(a) PCA: none

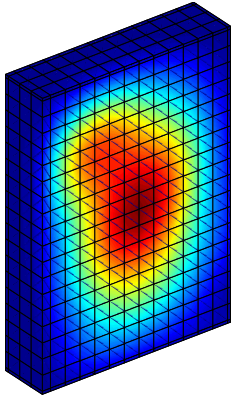

(b) PCA: source

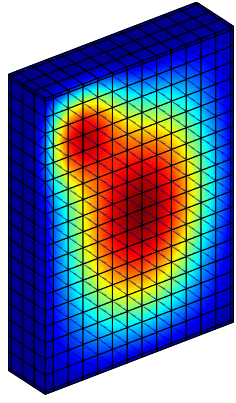

(c) PCA: field

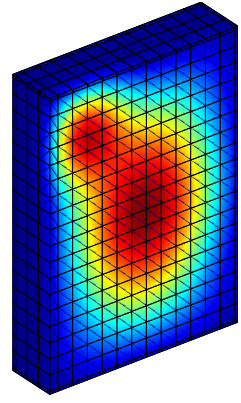

(d) PCA: both

Figure 6.8: Method (1). Absolute value of the reconstructed sound intensity (500Hz). Colors are scaled as in the exact solution (figure 6.4(a))

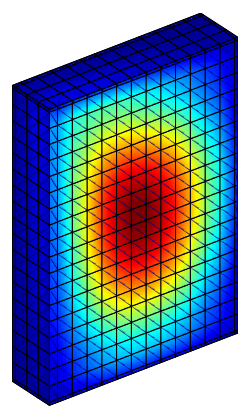

(a) Method PCA: none

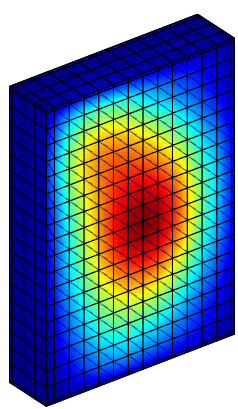

(3), (b) Method PCA: source

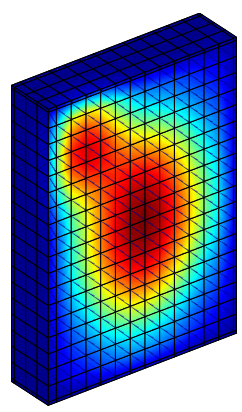

(3), (c) Method PCA: field

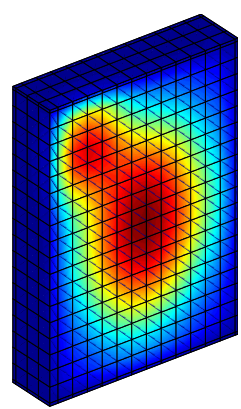

(3), (d) Method (3), PCA: both

Figure 6.9: Method (3). Absolute value of the reconstructed sound intensity (500Hz). Colors are scaled as in the exact solution (figure 6.4(a))

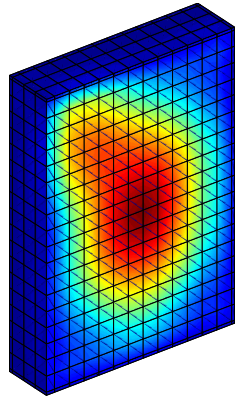

(a) New, none
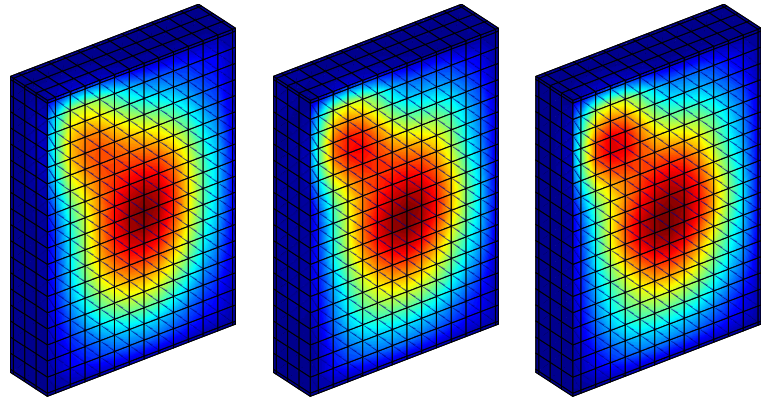

(d) New, PCA: both source

Figure 6.10: New method. Absolute value of the reconstructed sound intensity (500Hz). Colors are scaled as in the exact solution (figure 6.4(a)) 


\subsubsection{Experimental study}

The measurements data of the hard disk drive are used to test the accuracy of the inverse techniques. A detailed description of the measurement setup is given in section 4.4.3. The current section considers the auto spectrum of the velocity at the source, whereas the real part of the velocity has been discussed in chapter 4. The results of the new method is compared to the result of method (4) and laser-vibrometer data in figure 6.11 . The frequency is $9669 \mathrm{~Hz}$ and the point source clearly present in each of the three figures and the two inverse solutions are similar, which is in accordance with the simulation study. Given the results of the simulation study and the experimental study, it can be concluded that the new regularization method is similar to the existing methods.

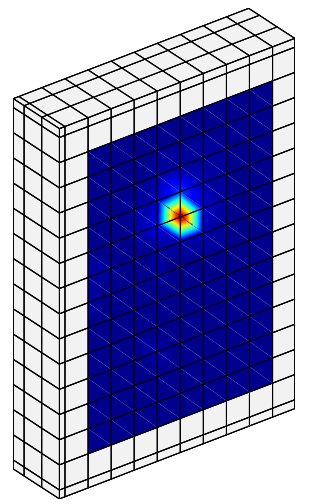

(a) Laser

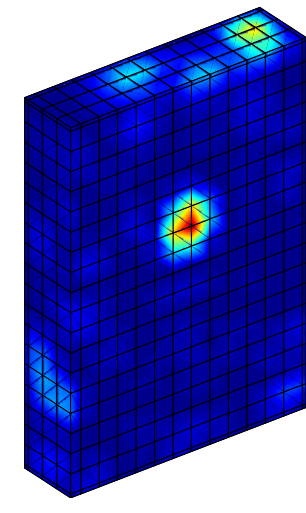

(b) Method (4)

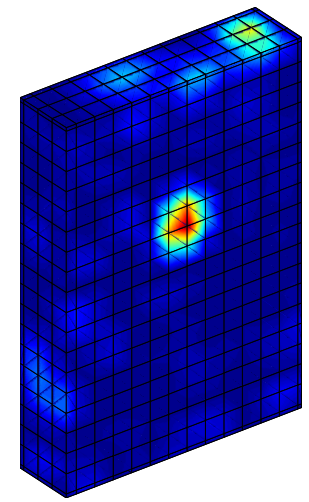

(c) New

Figure 6.11: Velocity autospectrum obtained in the experimental study (9669Hz)

\subsection{Point-to-surface cross spectra}

It is a challenge to gain an understanding of an acoustic system which exhibits multiple uncorrelated sources. To arrive at a clear understanding of the results, it is often insufficient to visualize the auto spectrum of the velocity and the sound intensity because these plots do not convey information about the correlation between different sources. This section considers a visualization method by Nam et al. [47], which can be applied to the existing methods as well as the new method.

A plot is made of the cross spectrum between the pressure or velocity at a single point $k$ and the velocity at the entire surface. This cross spectrum is termed a point-to-surface cross spectrum. The point is a BEM node which is selected by the user. If a node is selected whose contribution to the sound field is uncorrelated to 


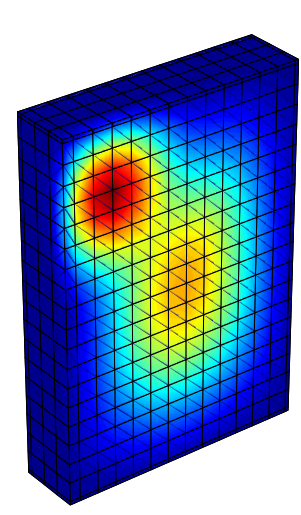

(a) Autospectrum

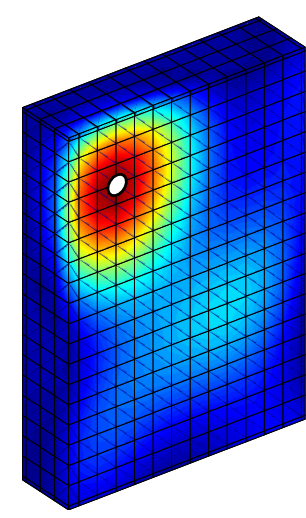

(b) (o) point 1

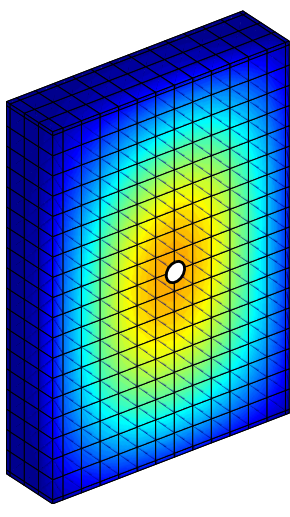

(c) (o) point 2

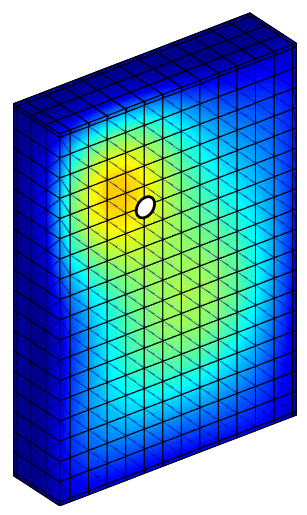

(d) (o) point 3

Figure 6.12: Autospectrum and point-to-surface cross spectra based on several points (absolute value, $500 \mathrm{~Hz})$ )

all other points, the point-to-surface cross spectrum is nonzero at node $k$ only. If there are other sources which also contribute in a correlated way, then the pointto-surface cross spectrum has a nonzero value which is visualized at these nodes as well. A similar approach has been considered by authors in the localization of aeroacoustic noise. The coherence is plotted, contrary to the cross spectrum [49].

To formulate the problem mathematically, let the vector $\mathbf{s}$ consist of the velocity at all source nodes and let $\mathbf{t}$ denote the scalar-valued velocity at node $k$. Hence, the cross spectrum $\mathbf{S}_{\mathbf{s t}} \in \mathbb{C}^{\hat{n} \times 1}$ must be calculated. A practically useful approach is to calculate the spectral matrix of the source $\mathbf{S}_{\mathbf{s s}} \in \mathbb{C}^{\hat{n} \times \hat{n}}$ and use the column corresponding to node $k$ as a post-processing step.

To show the usefulness of the point-to-surface cross spectra, the following example is considered. The model of the hard disk drive (see section 3.4) is used, the frequency is $500 \mathrm{~Hz}$ and the shape of vibration has been introduced in section 6.4. To calculate the source spectrum matrix, the existing method of equation 6.17 is used.

Although the velocity auto spectrum contains two clear peaks, it does not provide any information about uncorrelated effects (see figure 6.12(a)). By plotting the point-to-surface cross spectrum using the velocity at point 1 , it becomes clear that the velocity at the top-left peak is uncorrelated to the other vibrations (see figure 6.12(b)). Furthermore, the smooth shape at the middle of the front panel can be visualized using the point-to-surface cross spectrum using point 2 (see figure $6.12(\mathrm{c})$ ). The vibration of most nodes are partially correlated to both sources. For example, the point-to-surface cross spectrum based on point 3 contains both 
shapes of vibration (see figure 6.12(d)).

Given the results in figure 6.12, it can be concluded that point-to-surface cross spectra, originally proposed by Nam et al, are a useful tool to visualize multiple uncorrelated effects.

\subsection{DAMAS}

This section shows the relation between the DAMAS method in aeroacoustic source localization and the new approach which has been presented in this chapter. The DAMAS method is used to localize acoustic sources in wind tunnel tests [8]. Contrary to conventional beamforming which is more commonly used to localize aeroacoustic sources, the method treats source localization as a regularization problem.

Since the DAMAS method is aimed at acoustic source localization based in wind tunnels, the physical model is different from the models considered in this thesis. This leads to different transfer matrices but it does not change the inverse calculation conceptually. A second difference is that the sources at different points are assumed to be uncorrelated such that the spectral matrix of the source is then diagonal. The relation between the spectral matrix of the field and the spectral matrix of the source follows from equation 6.3.

$$
\begin{aligned}
\mathbf{S}_{\mathbf{f f}} & =\mathbf{H}_{\mathbf{f s}} \mathbf{S}_{\mathbf{s s}} \mathbf{H}_{\mathbf{f s}}^{H} \\
& =\mathbf{H}_{\mathbf{f s}} \operatorname{diag}(\mathbf{x}) \mathbf{H}_{\mathbf{f s}}^{H} \quad \text { with } \quad x_{k}=\left(\mathbf{S}_{\mathbf{s s}}\right)_{k k} \\
\left(\mathbf{S}_{\mathbf{f f}}\right)_{i j} & =\sum_{k=1}^{m}\left(\mathbf{H}_{\mathbf{f s}}\right)_{i k}\left(\overline{\mathbf{H}}_{\mathbf{f s}}\right)_{j k} x_{k}
\end{aligned}
$$

Defining $\left(\mathbf{H}_{\mathbf{s f}}\right)_{k}$ as the $k$ th column of the transfer matrix $\mathbf{H}_{\mathbf{s f}}$

$$
\begin{aligned}
\operatorname{vec}\left(\mathbf{S}_{\mathbf{f f}}\right) & =\left[\begin{array}{lll}
\left(\overline{\mathbf{H}}_{\mathbf{f s}}\right)_{1} \otimes\left(\mathbf{H}_{\mathbf{f s}}\right)_{1} & \cdots & \left(\overline{\mathbf{H}}_{\mathbf{f s}}\right)_{m} \otimes\left(\mathbf{H}_{\mathbf{f s}}\right)_{m}
\end{array}\right]\left\{\begin{array}{c}
x_{1} \\
\vdots \\
x_{m}
\end{array}\right\} \\
& =\mathbf{H} \mathbf{x}
\end{aligned}
$$

The DAMAS source localization problem consists of solving equation 6.42 for the unknown source strengths $\mathbf{x} \in \mathbb{C}^{\hat{n}}$, where $\mathbf{H} \in \mathbb{C}^{\hat{m}^{2} \times \hat{n}}$ is the relevant transfer matrix. An important difference between this inverse problem and most other problems considered in this thesis is the fact that the vector $\mathbf{x}$ does not contain negative elements because it consists of autospectra. To arrive at an inverse solution which cannot contain any negative elements, Brooks uses an iterative regularization method and sets the negative elements to zero in each iteration [8]. The regularization method is a slight variation on Landweber iteration [21]. 
Although the DAMAS method uses the same regularized inverse, the derivation given in this section is considered to be more direct than the derivation given by Brooks [8]. Appendix D shows that DAMAS is an efficient way to calculate the regularized inverse of the large matrix $\mathbf{H}$. Since the two methods are mathematically equivalent, they give the same results. Hence, an experimental comparison is not necessary.

\subsection{Rank of the calculated cross-spectral matrix}

It has become clear in the case study (section 6.4) that the existing methods are competitive with the new method if PCA is applied to the field data such that the rank of the field correlation matrix is exactly equal to the number of uncorrelated sources. It will be shown in this section that the rank of the source matrix cannot be larger than the rank of the field matrix if an existing method is used.

This is not true for the new method, which often calculates a source matrix with a higher rank. To understand this problem, consider the spectral matrix of the source in terms of singular values

$$
\mathbf{S}_{\mathbf{s t}}^{\prime}=\mathbf{U}_{\mathbf{f s}}^{H} \mathbf{S}_{\mathbf{s t}} \mathbf{U}_{\mathbf{g t}}
$$

For simplicity, assume $\mathbf{S}_{\mathbf{s t}}^{\prime}$ is a $2 \times 2$ matrix and that the exact source matrix has rank 1. Regularization based on the new method can cause only the auto-spectral term of the second singular value to be set to zero, such that the calculated source matrix is

$$
\mathbf{S}_{\mathbf{s t}}^{\prime}=\left[\begin{array}{cc}
S_{11} & S_{12} \\
S_{12} & 0
\end{array}\right]
$$

where the term $S_{j j}$ is zero due to regularization. If $S_{12}$ and $S_{21}$ are nonzero, this matrix has full rank even if the exact source matrix has rank one. This argument can be extended for larger matrices. The rank of the calculated source cross-spectral matrix of the source is larger than the rank of the cross-spectral matrix of the field. The error is caused by the regularization such that the errors diminish as the calculated source becomes more accurate.

It is interesting to consider the class of all inverse calculations which do not increase the rank. It can be seen that all methods which calculate the source matrix $\mathbf{S}_{\mathbf{s t}}$ as follows are part of this class.

$$
\mathbf{S}_{\mathbf{s t}}=\mathbf{A S}_{\mathbf{f g}} \mathbf{B}^{H}
$$

This includes all of the the existing methods (see equations 6.8, 6.12 and 6.16). The class of all linear operations which keep the rank constant has been reported on 
by Beasley [3]. In appendix $\mathrm{C}$ of this thesis, the class of all inverse calculations which do not increase the rank is studied, while making use of the assumption that the inverse method applies a filter to the singular values of the transfer matrix of equation 6.25

$$
\operatorname{vec}\left(\mathbf{S}_{\mathbf{s t}}\right)=\mathbf{V} \mathbf{\Sigma}^{\dagger \alpha} \mathbf{U}^{H} \operatorname{vec}\left(\mathbf{S}_{\mathbf{f g}}\right)
$$

where $\mathbf{U}$ and $\mathbf{V}$ are the singular vectors of the transfer matrix in accordance with equation 6.25. This includes the new method, the existing method of equation 6.16 as well as many other methods. The result of appendix $C$ is that all inverse operations of the form of equation 6.46 which do not increase the rank can also be written as equation 6.45 .

These mathematical considerations can be interpreted as follows. If the number of uncorrelated sources is known, it is intuitive to use an inverse technique which does not increase the number of uncorrelated sources. If the inverse method has the form of equation 6.46, then the inverse method must be of the form of equation 6.45. In a sense, $\mathbf{A}$ and $\mathbf{B}$ are regularized inverses $\mathbf{H}_{\mathrm{fs}}$ and $\mathbf{H}_{\mathrm{gt}}$ respectively. Hence, the existing method (3) (equation 6.16) is considered to be a natural choice in this class of methods.

\subsection{Summary}

This chapter has presented a new method to apply inverse acoustics to cross spectra. These methods are useful to calculate the autospectrum of the source velocity, point-to-surface cross spectra and most importantly, the sound intensity.

A disadvantage of the new method is the fact that the number of calculated uncorrelated sources is often too large. Although a matrix which has the correct rank can be calculated by applying PCA to the source matrix, the existing methods are considered a more natural way to avoid this problem.

A theoretical framework has been presented. Both the new and the existing methods have a natural place in the presented framework. Additionally, the DAMAS method in aeroacoustic source localization has been explained using the same theory. A simulation study indicates that the new and existing methods provide similar results if PCA is applied to the field data but the new methods improve the result if PCA is not applied. 


\section{Chapter 7}

\section{Two BEM-based point-source localization methods}

\subsection{Introduction}

This chapter considers the localization of point sources on an arbitrarily shaped surface. Contrary to the other inverse acoustic methods, this problem is well conditioned. It will be shown by means of simulations that this makes it possible to localize a source even if the measurement data contains twice as much signal as noise. To develop a point-source localization technique for arbitrary surfaces, a well known technique to localize plane waves (MUSIC) is applied to the boundary element method (BEM). This method is compared to a straightforward leastsquares approach.

In practice, these methods can be used to localize acoustic leaks in various applications such as car-interior noise and building acoustics. After the location of the sources has been determined, their strengths can be calculated as a postprocessing step (see for example [2]).

This chapter is built up as follows. Section 7.2 describes the application of the widely used MUSIC method to BEM-based point-source localization. Section 7.3 considers a least squares approach. Section 7.4 shows the relation between pointsource localization and regularization. A simulation study as well as an experimental validation are considered in section 7.5 and a summary is given in section 7.6.

\subsection{MUSIC}

The first method which is used to localize point sources is MUSIC (MUltiple SIgnal Classification) [72]. It is widely applied in electromagnetism and acoustics to 
solve direction-of-arrival problems [40,54]. In these applications, it is used to identify the direction of incidence of multiple plane waves based on measurement data from an array of sensors. The results are plotted in the form of the MUSIC spectrum, which is a function of the angle of incidence contains sharp peaks at the angles of incidence. From this plot, it is easy to see from which angles the waves have arrived. The number of sources that can be localized is at most $\hat{m}-1$, where $\hat{m}$ is the number of sensors. If the number of sources is large, the problem can become ill-conditioned such that the problem cannot be solved accurately (see for example [102]). Although the MUSIC method is often based on an analytical physics model, a BEM model is used in this chapter to localize point sources on arbitrarily shaped surfaces in the acoustic near field.

To explain the MUSIC method, it is assumed that the sound field is caused by velocities at a number of nodes. This number is denoted as $k$. The source locations are denoted $\vec{y}_{j}, j \in\{1,2, \cdots, k\}$, and all other points are assumed to have a velocity of zero. Furthermore, the sources are assumed not to be fully correlated. Let $\mathbf{s}(\omega) \in$ $\mathbb{C}^{k}$ and $\mathbf{f}(\omega) \in \mathbb{C}^{\hat{m}}$ denote deterministic source and field vectors in the frequency domain. Their relation is given by

$$
\begin{aligned}
{\left[\begin{array}{cccc}
\vdots & \vdots & & \vdots \\
\mathbf{h}\left(\vec{y}_{1}\right) & \mathbf{h}\left(\vec{y}_{2}\right) & \cdots & \mathbf{h}\left(\vec{y}_{k}\right) \\
\vdots & \vdots & & \vdots
\end{array}\right]\left\{\begin{array}{c}
s_{1} \\
s_{2} \\
\vdots \\
s_{k}
\end{array}\right\} } & =\left\{\begin{array}{c}
f_{1} \\
f_{2} \\
\vdots \\
f_{\hat{m}}
\end{array}\right\} \\
\mathbf{H}_{\mathbf{f s}} \mathbf{s} & =\mathbf{f}
\end{aligned}
$$

where $\mathbf{h}\left(y_{j}, \omega\right) \in \mathbb{C}^{\hat{m} \times 1}$ denotes the transfer function from the velocity at point $\vec{y}_{j}$ to all of the field points. This transfer function is a column of a BEM transfer matrix in the current context and the Rayleigh integral applies if the sources are located on a planar surface. Since none of the sources are fully correlated, the signal subspace of $\mathbf{f}$ is the entire range of $\mathbf{H}_{\mathbf{f s}}$. This implies that each column $\mathbf{h}\left(\vec{y}_{j}\right), j \in\{1,2 \ldots \hat{m}\}$ is an element of the signal subspace. A point $\vec{y}_{i}$ can therefore only be a source if $\mathbf{h}\left(\vec{y}_{i}\right)$ is an element of the signal subspace. The signal subspace can be calculated from measurement the using PCA (see section 5.3.3) if the number of uncorrelated sources $k$ is known.

The MUSIC algorithm goes through each node one by one. The node location is denoted as $\vec{y}$. For each node, it determines the distance $\delta(\vec{y})$ in $\hat{m}$ dimensional space between the transfer function $\mathbf{h}(\vec{y})$ and the experimentally determined signal subspace. To visualize the result the MUSIC spectrum is the function $1 / \delta(\vec{y})^{2}$. It is displayed as a color plot on the BEM mesh in this chapter. Since it contains peaks at locations where the model of a point source at $\vec{y}$ and the signal subspace is small, it is a useful visual tool to localize the sources. If a source is located at $\vec{y}$, then 
the signal subspace and the transfer function $\mathbf{h}(\vec{y})$ are close such that the MUSIC spectrum exhibits a peak. The opposite is also true for many relevant problems: if the signal subspace and the transfer function $\mathbf{h}(\vec{y})$ are close, then there is a source at $\vec{y}$, but there are exceptions to this second observation.

The distance between the signal subspace and the transfer function $\mathbf{h}(\vec{y}, \omega)$ can be calculated by noting that this distance is (by definition) the minimum distance between the point $\mathbf{h}(\vec{y})$ and any point in the signal subspace:

$$
\delta(\vec{y})=\min _{\mathbf{w}}\left\|\mathbf{h}(\vec{y})-\mathbf{\Phi}_{S} \mathbf{w}\right\|
$$

where $\boldsymbol{\Phi}_{S}$ is an orthonormal basis of the noise subspace. A $2 \mathrm{D}$ example of this equation is depicted in figure 7.1(a). The following, equivalent, definition of the distance is commonly found in the literature.

$$
\delta(\vec{y})=\left\|\boldsymbol{\Phi}_{N}^{H} \mathbf{h}(\vec{y})\right\|
$$

Where $\boldsymbol{\Phi}_{N}$ is an orthonormal basis of the signal subspace. As stated above, the MUSIC spectrum is useful to determine the distances. It is given by

$$
S_{\text {MUSIC }}(\vec{y})=\frac{1}{\delta(\vec{y})^{2}}
$$

It can be seen that this function increases rapidly as the distance $\delta(\vec{y})$ becomes small and even tends to infinity if the distance tends to zero.

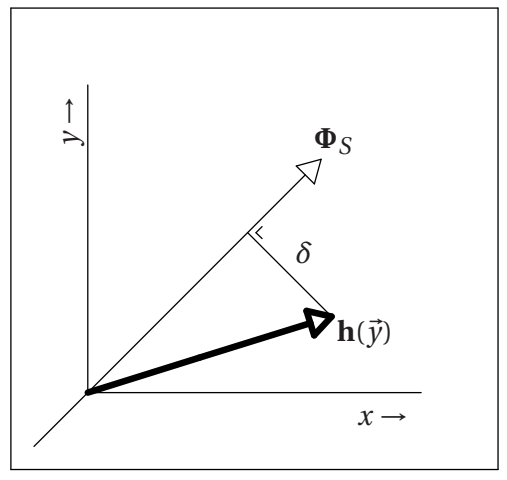

(a) Distances in accordance with equations 7.3 and 7.4

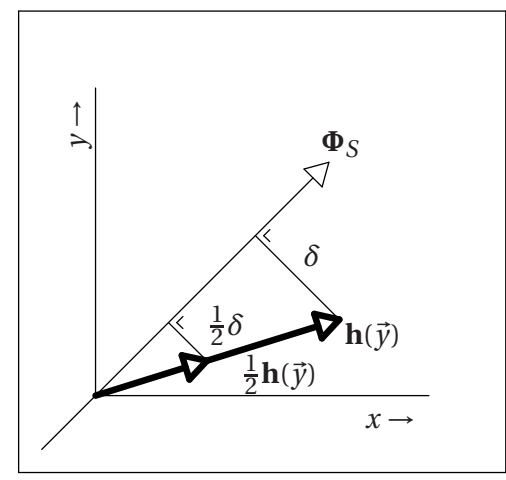

(b) Scaling the transfer function

Figure 7.1: 2D Examples of the distances between the transfer function and the signal subspace 
An important aspect of the implementation is that the distance in equation 7.4 depends on the norm of the transfer function $\mathbf{h}(\vec{y}, \omega)$ (see figure 7.1(b)). Erroneous peaks are visible in the MUSIC spectrum at those nodes where the norm of $\mathbf{h}(\vec{y}, \omega)$ is small. This problem is solved by scaling each transfer function to have unit norm.

\subsection{A least squares approach}

A disadvantage of the MUSIC spectrum is the fact that the source locations are considered sequentially. Hence, it only considers how well a single point source at location $\vec{y}$ explains the field data but it does not consider how well other sources can explain the remaining field data. A straightforward least squares problem does provide this information and it is also suitable to localize sources if they are correlated. The measured dataset is represented by the matrix $\mathbf{F} \in \mathbb{C}^{\hat{m} \times l}$, which contains $l$ STFT coefficients of the $\hat{m}$ sensors. The distance between the model and the field data is defined as follows.

$$
\delta\left(\vec{y}_{1}, \vec{y}_{2}, \cdots, \vec{y}_{k}\right)=\min _{\mathbf{S} \in \mathbb{C}^{k \times l}}\left\|\left[\begin{array}{llll}
\mathbf{h}\left(\vec{y}_{1}\right) & \mathbf{h}\left(\vec{y}_{2}\right) & \cdots & \mathbf{h}\left(\vec{y}_{k}\right)
\end{array}\right] \mathbf{S}-\mathbf{F}\right\|
$$

This is a standard least squares problem which can be solved using the pseudoinverse of $\left[\begin{array}{lll}\mathbf{h}\left(\vec{y}_{1}\right) & \mathbf{h}\left(\vec{y}_{2}\right) & \cdots \mathbf{h}\left(\vec{y}_{k}\right)\end{array}\right]$. Since $\delta$ is a function of multiple locations, it cannot be visualized as a function of a single location $\vec{y}$. Since only small values of the residual norm are relevant, the following function of $\vec{y}$ gives a good impression of the agreement between the model and the measurement data if one of the sources is located at $\vec{y}$

$$
\delta(\vec{y})=\min _{\vec{y}_{2}, \cdots, \vec{y}_{k}} \delta\left(\vec{y}, \vec{y}_{2}, \cdots, \vec{y}_{k}\right)
$$

It can be shown that this definition of distance is equal to the distance in the MUSIC spectrum up to a constant factor if the number of sources $k$ and the number of averages $l$ are 1 and furthermore, if the transfer function is scaled to have unit norm. Otherwise, the results are different. To visualize equation 7.7 , the least squares spectrum is defined in the same way as the MUSIC spectrum

$$
S_{\text {Least squares }}(\vec{y})=\frac{1}{\delta^{2}}
$$

The maximum value of $S_{\text {Least squares }}(\vec{y})$ usually occurs at $k$ locations. The locations at which this maximum occurs are the parameters $\vec{y}_{1}, \vec{y}_{2}, \cdots, \vec{y}_{k}$ which minimize equation 7.6. 
Equation 7.7 is a non-quadratic optimization problem in $k-1$ variables. In the case study of this section, the optimum is calculated by calculating all possible values of $\delta\left(\vec{y}_{1}, \vec{y}_{2}, \cdots, \vec{y}_{k}\right)$ where $\vec{y}_{1}, \vec{y}_{2}, \cdots, \vec{y}_{k}$ are node locations. This is a timeconsuming process. In the case study, the localization of two sources required 68 seconds, compared to 1.3 seconds for the MUSIC algorithm. Both calculations have been performed using optimized MATLAB code on a $3 \mathrm{GHz}$ PC with 1GB of RAM. The source mesh consists of 2100 nodes and two uncorrelated sources are used. More advanced algorithms will undoubtedly reduce the computation time.

\subsection{Relation to regularization}

The point-source localization methods of this chapter do not require regularization. This section shows that the assumption that only $k$ sources exist is mathematically similar to the stabilization of Tikhonov regularization.

Tikhonov regularization has been introduced in this thesis as a filter on the singular values (see equation 3.9) but it can also be expressed as a minimization of the Tikhonov functional [21]

$$
\widehat{\mathbf{s}}=\arg \min _{\widetilde{\mathbf{s}}}\left\|\mathbf{H}_{\mathbf{f s}} \widetilde{\mathbf{s}}-\widehat{\mathbf{f}}\right\|^{2}+\lambda^{2}\|\widetilde{\mathbf{s}}\|^{2}
$$

where argmin denotes the argument of the minimum: the value of $\widetilde{\mathbf{s}}$ which minimizes the Tikhonov functional. This optimization problem can be derived by applying the Kuhn-Tucker conditions to the following optimization problem [42]

$$
\widehat{\mathbf{s}}=\arg \min _{\widetilde{\mathbf{s}}}\left\|\mathbf{H}_{\mathbf{f s}} \widetilde{\mathbf{s}}-\widehat{\mathbf{f}}\right\|^{2} \quad \text { subject to }\|\widetilde{\mathbf{s}}\|^{2} \leq \rho^{2}
$$

where $\rho$ acts as the regularization parameter. It can be seen that equation 7.10 is a minimization of the residual norm, with a constrained solution norm.

The least squares problem of equation 7.6 is a minimization of the solution norm with a constrained number of point sources. If the number of averages $l$ is one, it can be expressed as

$$
\begin{aligned}
& \delta=\quad \min _{\widetilde{\mathbf{s}}}\left\|\mathbf{H}_{\mathbf{f s}} \widetilde{\mathbf{s}}-\widehat{\mathbf{f}}\right\|^{2} \quad \text { subject to } \quad \# \widetilde{\mathbf{s}} \leq k \\
& \widehat{\mathbf{s}}=\arg \min _{\widetilde{\mathbf{s}}}\left\|\mathbf{H}_{\mathbf{f s}} \widetilde{\mathbf{s}}-\widehat{\mathbf{f}}\right\|^{2} \quad \text { subject to } \quad \# \widetilde{\mathbf{s}} \leq k
\end{aligned}
$$

where $\# \widetilde{\mathbf{s}}$ denotes the number of nonzero elements in the vector $\widetilde{\mathbf{s}}$. The calculated source vector $\widetilde{\mathbf{s}}$ is a stable solution to the point-source localization problem, such that the only theoretical difference between point-source localization and conventional IBEM is the constraint used. 


\subsection{Case study}

A simulation study is performed using the model of the hard disk drive (see section 3.4). The frequency is $500 \mathrm{~Hz}$. In the first case, a single point source is simulated at the top-left of the hard disk drive and the number of averages $l$ is chosen to be one. This means that MUSIC is mathematically equivalent to the least squares method in this first case. The results are depicted in figure 7.2. It can be seen that the method is extremely robust to noise. A clear peak can even be seen if the signalto-noise ratio is $\frac{1}{2}$, such that the added noise has twice the norm of the exact field vector.

In the second case, two uncorrelated sources exist, a signal-to-noise ratio of $\frac{10}{3}$ is used and the source at the left top has half the amplitude of the source at the right bottom. The MUSIC spectrum is compared to the least squares method of equation 7.7 in figure 7.3. Both results have peaks at the correct nodes but the top-left peak in the MUSIC spectrum is roughly a quarter of the height of the bottom-right peak. The least-squares method has peaks of equal amplitude such that both peaks are clearly visible. This makes it straightforward to distinguish between sources of small amplitude and artifacts of the method. For this reason, the least squares method is considered superior. Figure 7.4 shows that the results are similar if the field grid is reduced from $17 \times 21$ to $4 \times 4$ points.

The methods are applied to the experimental data of the hard disk drive. At $9668 \mathrm{~Hz}$, the laser vibrometry results clearly indicate a point source at the front panel of the hard disk (see also chapter 4 figure 4.9 (b)). The experimental dataset does not contain multiple uncorrelated sources such that the MUSIC method and the least squares method give the same result. This result is depicted in figure 7.5(a). The least squares approach can also localize two correlated sources. The result is depicted in figure 7.5(b). This image suggests that the sound field is approximated well by a point source at the front panel as well as a point source at the top panel of the hard disk drive. It is possible that the combination of more point sources yields a more accurate representation of the sound field. If the field grid is reduced to $4 \times 4$ points, the source at the front panel is also localized accurately (see figure 7.6).

\subsection{Summary}

This chapter has applied the MUSIC method to BEM to localize point sources on arbitrarily shaped surfaces in the acoustic nearfield and the method has been compared to a straightforward least squares method.

A simulation study has shown that both methods yield usable results even if the field data contains twice as much noise as signal. At a less extreme noise level, the 

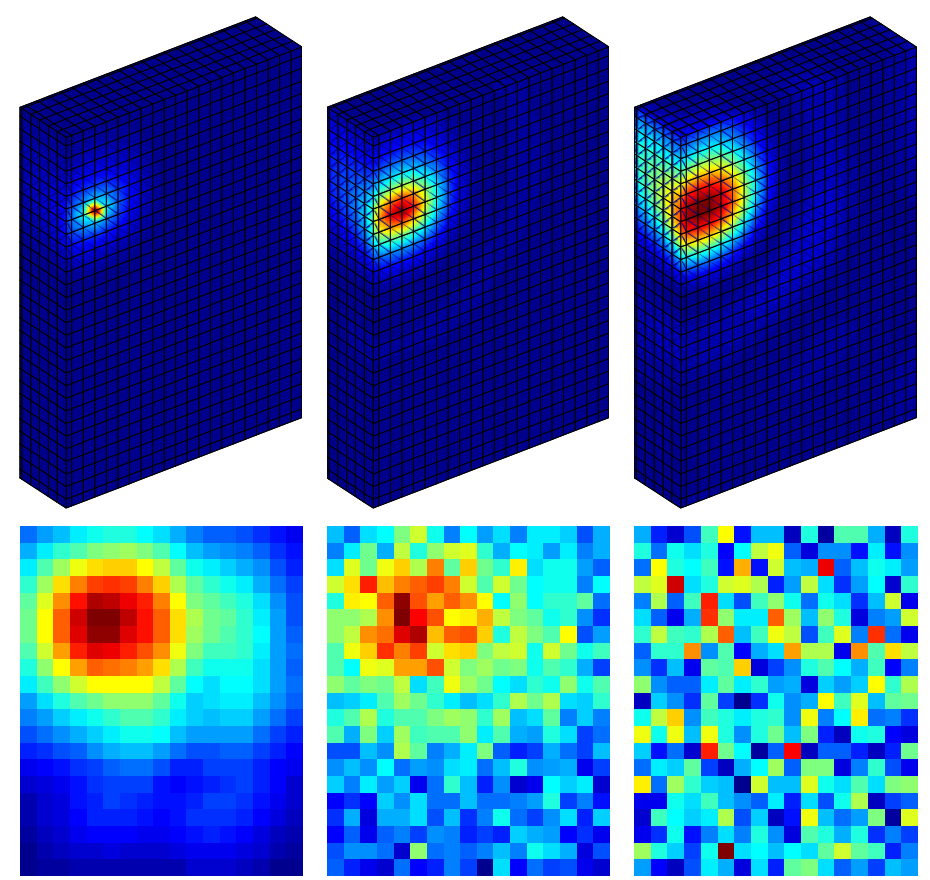

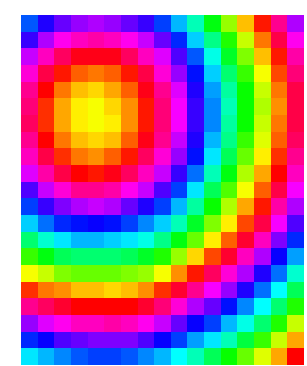

(a) $\mathrm{SNR}=100$

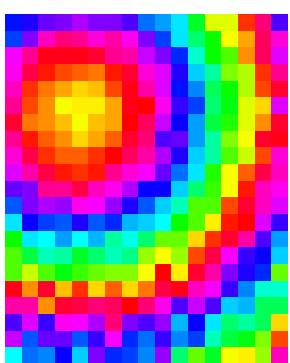

(b) $\mathrm{SNR}=\frac{10}{3}$

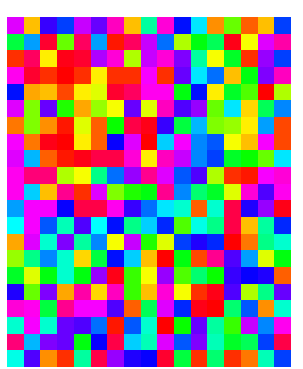

(c) $\mathrm{SNR}=\frac{1}{2}$

Figure 7.2: Simulation results of MUSIC using a single source based on a signal-to-noise ratio (SNR) of $100, \frac{10}{3}$ and $\frac{1}{2}$ respectively. Top: MUSIC spectrum, middle: field (absolute value), bottom, field (phase). 


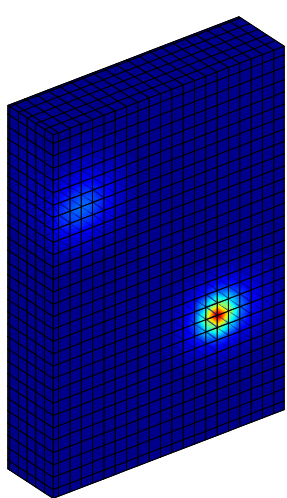

(a) MUSIC

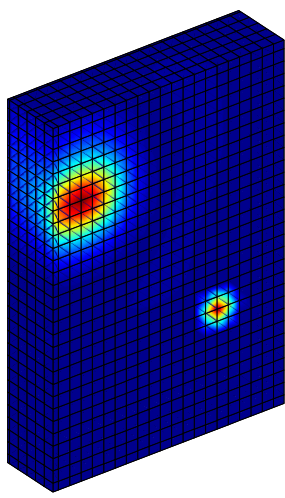

(b) Least squares

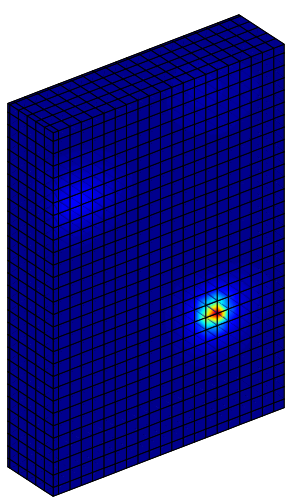

(b) MUSIC

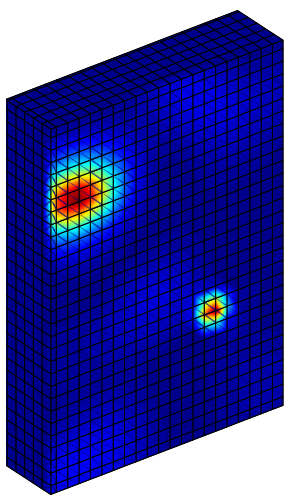

(b) Least squares

Figure 7.3: Simulated MUSIC and Least squares spectrum two sources based on 357 field points $(500 \mathrm{~Hz})$.

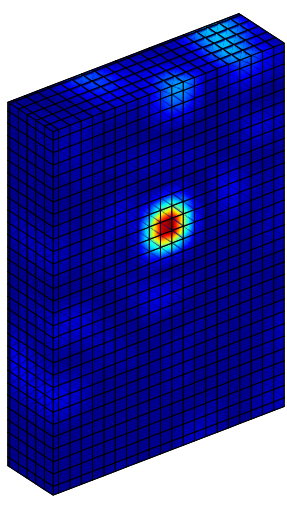

(a) MUSIC

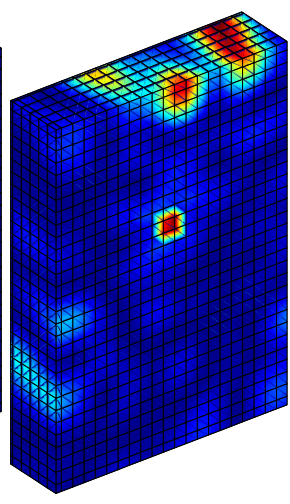

(b) Least squares

Figure 7.5: Experimental MUSIC and Least squares spectrum two sources based on 357 field points (9668Hz).

Figure 7.4: Simulated MUSIC and Least squares spectrum two sources based on 16 field points $(500 \mathrm{~Hz})$.

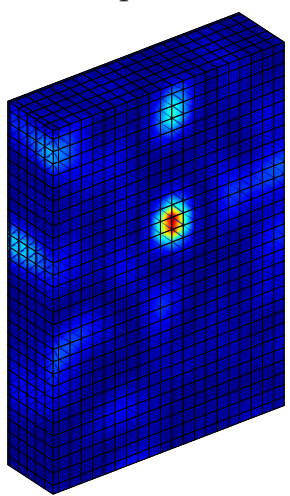

(b) MUSIC

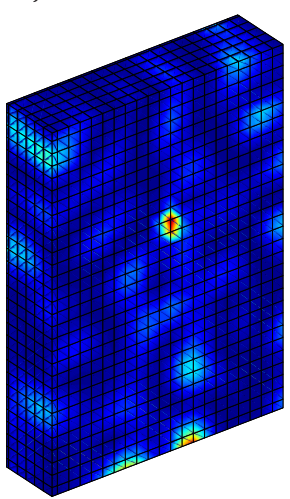

(b) Least squares
Figure 7.6: Experimental MUSIC and Least squares spectrum two sources based on 16 field points $(9668 \mathrm{~Hz})$. 
sources also localized accurately if the field grid consists of $4 \times 4$ points.

The main advantage of the least squares method is the fact that the largest peaks all have the same height, such that it is straightforward to distinguish between side-lobes of the main source and actual sources. The main disadvantage of the algorithm which has been used is the fact that its order is $O\left(\hat{n}^{k}\right)$, where $\hat{n}$ and $k$ are the number of nodes of the BEM model and the number of sources respectively. Hence, the localization of more than two sources over a range of frequencies is computationally costly.

The advantage of the MUSIC method is its speed and its disadvantage is the fact that the peaks can have different heights. The least squares method is therefore considered to be more attractive provided that the computation time is acceptable. 


\section{Chapter 8}

\section{Conclusions and Recommendations}

\subsection{Conclusions}

This thesis considers the theoretical and practical aspects of acoustic source localization. Insights from engineering and applied mathematics are used to gain an understanding of acoustic source localization problems and to solve them efficiently. A wide range of topics is covered, varying from the study of regularization methods for cross-spectral matrices to signal processing techniques for moving sensors. Although the theory of regularization is the same for each of these topics, differences between the forward models lead to different challenges and opportunities. The following conclusions can be drawn with respect to each of these topics.

- Based on the literature in applied mathematics, the Lanczos-SVD algorithm is newly applied to inverse acoustic problems. In its current application, it is a fast alternative to the conventional Goulub-Kahan SVD algorithm.

- The theory and algorithms for Toeplitz matrices are applied to the planar inverse acoustic problem. This new approach yields results which are as robust and accurate as statistically optimized nearfield acoustic holography (SONAH) at a reduced computational cost. The advantages are especially pronounced for large-scale problems and the method is even applicable in cases where the transfer matrix is too large to be stored in computer memory explicitly. It has also been shown that PNAH is based on circulant matrices whereas the new method is based on the more general class of Toeplitz matrices. The new approach has been implemented in a software package named 
iTrim.

- It is shown that conventional inverse acoustic methods such as the inverse boundary element method (IBEM) can be applied to a measurement setup where a sensor moves through the sound field continuously, contrary to fullarray or point-by-point measurements. To arrive at an accurate representation of the field data, the transfer function from the reference sensor to the field sensor is calculated using the multi-taper method. It is shown experimentally that this method gives accurate results.

- A new method to apply inverse acoustics to cross spectra has been presented. These methods are useful to calculate the auto spectrum of the source velocity, point-to-surface cross spectra and most importantly, the sound intensity. Within the theoretical framework of this method, it is also possible to analyze a number of existing methods in nearfield source localization as well as the DAMAS method in aeroacoustic source localization. A case study shows that the new method improves the accuracy by $30 \%$ if principal component analysis (PCA) is not applied to the field data, and that the accuracy is similar otherwise. A software package named CrossReg has been developed. This package implements the new and existing techniques and the forward model can consist of BEM as well as the new approach based on Toeplitz matrices.

- The MUSIC method is applied to BEM to localize point source in the acoustic near field. It is shown by means of both simulations and experiments that this method can localize a point source even if the grid of field sensors is reduced to a grid of $4 \times 4$ points. In simulation, it has also been shown that the sources can be localized if the field data contains twice as much noise as signal.

\subsection{Recommendations}

Based on the knowledge gained in this study, the following aspects of further research are recommended.

To improve the accuracy, reliability and ease-of-use of acoustic of source localization techniques, improved measurement methods are considered to be essential. Further research can focus on improved sensors such as conventional transducers or optical systems, but it can also focus on the development and validation of a procedure to determine the location and orientation of a moving sensor in three-dimensional space along the lines of the Octopus digitizer proposed by Tijs et al. [82]. 
The new method based on Toeplitz matrices can be extended in several ways. It can be applied to cylindrical geometries, where the transfer matrix has a block Toeplitz with circulant blocks (BTCB) structure. Furthermore, other regularization methods can be applied, as discussed in section 4.3.5.

To develop fast inverse methods for arbitrary geometries, fast methods such as fast multipole BEM [18] or multilevel multi-integration schemes [66] are useful. Multi-level regularization methods are widely studied in the mathematical literature, see e.g. [37].

A considerable amount of information about the source vibrations can be found by comparing the field data at multiple frequencies. A preliminary exploration of this topic has been presented by Druyvesteyn, Ligtenberg and the current author in [14], where point sources are localized based on measurement data from multiple frequencies.

In 2008, Song proposed a method termed sound quality mapping, which is a beamforming method to identify sound sources in terms of their psychoacoustic attributes such as loudness and sharpness [78]. Further research can be carried out on to the application of this approach to nearfield inverse acoustics to localize the strength and psychoacoustic quality of the acoustic sources. 


\section{Appendix A}

\section{An exact description of the sound at the field points}

\section{Introduction}

An interesting theoretical result is the fact that a set of $m$ shape functions exist which achieves a discretization error of zero for the Rayleigh integral with a finite number $m$ of field points. Although the entire sound field can not be made exact using only $m$ shape functions, the error can be made zero at all of the sensors. A set of shape functions that achieves this goal is as follows

$$
N_{j}(\vec{y})=\frac{e^{\mathrm{i} k\left|\vec{y}-\vec{x}_{j}\right|}}{\left|\vec{y}-\vec{x}_{j}\right|}
$$

where the solution is exact if the discrete velocities $v_{j}$ are determined based on an arbitrary function $v(\vec{y})$ in the sense of the least squares over a bounded surface $S$.

$$
\left\{v_{1}, v_{2}, \ldots\right\}=\arg \min _{\left\{\nu_{1}, \nu_{2}, \ldots\right\}} \int_{S}\left|\sum_{j} N_{j}(\vec{y}) v_{j}-v(\vec{y})\right|^{2} d S
$$

Where arg min denotes the argument of the minimum: the vector of velocities that achieves the minimum distance. Although this result is interesting from a theoretical point of view, element methods are more practical. Firstly, to calculate the field pressures based on a known velocity function, it is usually necessary to discretize equation A.2. In that case, interpolation errors are unavoidable. This problem does not occur in inverse problems, where the continuous source velocity is calculated from the discrete velocities using only equation A.1.

A second practical advantage of element methods is that their shape functions are from linearly dependent and two basis functions are even orthogonal if their 
nodes are not part of the same element. In contrast, the shape functions in equation A.1 can become almost linearly dependent which can cause numerical problems.

Thirdly, the matrix coefficients of element methods can be calculated as integrals over small areas whereas the numerical integrals in equation A.2 must be taken over the entire area. This can make a decisive difference in the computational cost.

The last two drawbacks are not exclusive to equation A.1: they have also been reported for a number of methods based where the shape functions are Bessel functions or complex exponentials [60].

\section{Derivation}

Equation A.1 is the transpose of the linear operation defined by the Rayleigh integral after a proper formalization of the problem.

Let $s \in L_{2}(S)$ and $\mathbf{f} \in \mathbb{C}^{m}$ be the velocity function on the source plane and the vector containing pressures at the measurement points respectively. Furthermore, let $\Gamma$ denote a finite source area. The transfer from source velocity to field pressure is given by the Rayleigh integral, which is a linear operation

$$
\mathbf{f}=H s
$$

where $H_{\mathbf{f} s}: L_{2}(S) \rightarrow \mathbb{C}^{m}$ is a linear operation representing the Rayleigh integral. The discrete form of equation A.3 can be defined by introducing a set of shape functions $v_{j} \in L_{2}(S), j \in\{1,2, \ldots, m\}$

$$
\begin{aligned}
\widehat{\mathbf{f}} & =\sum_{i=1}^{m} H v_{i} s_{i} \\
& =H V \mathbf{s} \quad \text { where } \quad V: V \mathbf{s}=\sum_{i=1}^{m} v_{i} s_{i}
\end{aligned}
$$

where $\widehat{\mathbf{f}}$ is the solution of the discrete problem, $\mathbf{s}=\left\{s_{1}, s_{2}, \ldots, s_{m}\right\}^{T} \in \mathbb{C}^{m}$ is the vector of discrete source velocities.

The source velocities are determined in the sense of the least squares

$$
\begin{aligned}
& \mathbf{s}=\arg \min _{\tilde{\mathbf{s}}}\|V \tilde{\mathbf{s}}-s\| \\
& \mathbf{s}=\left(V^{H} V\right)^{-1} V^{H} s
\end{aligned}
$$


The set of shape functions $V=H^{H}$ achieves a discretization error of zero. This can be demonstrated by combining equation A.5 and A.7

$$
\begin{aligned}
\widehat{\mathbf{f}} & =H V\left(V^{H} V\right)^{-1} V^{H} s \\
& =H H^{H}\left(H H^{H}\right)^{-1} H s \\
& =H s=\mathbf{f}
\end{aligned}
$$

It can be seen that a discretization error of zero is achieved, provided that $H$ is injective. The final step is to find an analytical expression for $H^{H}$. The solution is

$$
\left(H^{H} \mathbf{f}\right)(\vec{y})=\frac{\mathrm{i} \rho_{0}}{2 \pi} \sum_{i} \frac{e^{\mathrm{i} k\left|\vec{y}-\vec{x}_{i}\right|}}{\left|\vec{y}-\vec{x}_{i}\right|} f_{i}
$$

Note that the argument of the complex exponential has opposite sign compared to equation the Rayleigh integral which is given by (see equation 2.12).

$$
H s=\frac{\mathrm{i} \rho_{0}}{2 \pi} \int_{S} \frac{e^{-\mathrm{i} k\left|\vec{y}-\vec{x}_{i}\right|}}{\left|\vec{y}-\vec{x}_{i}\right|} s(\vec{y}) d S
$$

Where $\vec{x}_{i}, i \in\{1,2, \ldots, m\}$ is the set of measurement points. To show that equation A.11 is indeed the transpose of equation A.12, the definition of the transpose is simply plugged into equation A.12. This definition is

$$
\langle H s, f\rangle=\left\langle s, H^{H} f\right\rangle
$$

Combining equations A.11, A.12 and A.13, we have

$$
\begin{aligned}
\left\langle s, H^{H} f\right\rangle & =\int_{\Gamma} s(\vec{y}) \cdot \overline{\left(H^{H} \mathbf{f}\right)(\vec{y})} d \Gamma \\
& =\frac{\mathrm{i} \rho_{0}}{2 \pi} \int_{\Gamma} s(\vec{y}) \overline{\sum_{i} \frac{e^{\mathrm{i} k\left|\vec{y}-\vec{x}_{i}\right|}}{\left|\vec{y}-\vec{x}_{i}\right|} f_{i} d \Gamma} \\
& =\frac{\mathrm{i} \rho_{0}}{2 \pi} \sum_{i} \int_{\Gamma} \frac{e^{-\mathrm{i} k\left|\vec{y}-\vec{x}_{i}\right|}}{\left|\vec{y}-\vec{x}_{i}\right|} s(\vec{y}) d \Gamma \cdot \bar{f}_{i} \\
& =\sum_{i}(H s)_{i} \cdot \bar{f}_{i}=\langle H s, f\rangle
\end{aligned}
$$

which proves equation A.11. It follows that equation A.1 is a basis of functions which achieves a discretization error of zero if $H$ is injective. 


\section{Appendix B}

\section{Total least squares}

This appendix shows that minimizing the perpendicular distance to a line is an eigenvalue problem. The eigenvalue problem is derived and its relation to equation 5.45 is clarified.

Let each of the rows of the matrices $\widetilde{\mathbf{F}} \in \mathbb{C}^{\hat{m} \times l}$ and $\widetilde{\mathbf{G}} \in \mathbb{C}^{m \times l}$ contain $l$ STFT coefficients of the vector valued signals $\hat{F}(t)$ and $\hat{G}(t)$ at frequency $\omega$ respectively. The signals are assumed to be whitened such that they are perturbed by white noise. To approximate the transfer matrix, it is noted that there are matrices $\mathbf{F}$ and $\mathbf{G}$ which are close to $\widetilde{\mathbf{F}}$ and $\widetilde{\mathbf{G}}$ respectively such that the following relation holds.

$$
\mathbf{H}_{\mathrm{gf}} \mathbf{F}=\mathbf{G}
$$

The total least squares estimate finds a transfer matrix $\mathbf{H}_{\mathbf{g f}}$ such that equation B.1 holds exactly, and the squared distance between $\widetilde{\mathbf{F}}$ and $\mathbf{F}$ as well as the squared distance between $\widetilde{\mathbf{G}}$ and $\mathbf{G}$ is minimized. The squared distance is defined to be

$$
\begin{aligned}
\delta^{2} & =\sum_{i=1}^{\hat{m}} \sum_{j=1}^{l}\left|\mathbf{f}_{i j}-\widetilde{\mathbf{f}}_{i j}\right|^{2}+\sum_{p=1}^{m} \sum_{q=1}^{l}\left|\mathbf{g}_{p q}-\widetilde{\mathbf{g}}_{p q}\right|^{2} \\
& =\left\|\left[\begin{array}{l}
\mathbf{F} \\
\mathbf{G}
\end{array}\right]-\left[\begin{array}{c}
\widetilde{\mathbf{F}} \\
\widetilde{\mathbf{G}}
\end{array}\right]\right\|_{F}^{2}
\end{aligned}
$$

where $\|\cdot\|_{F}^{2}$ denotes the squared Frobenius norm, which is the sum of the squared absolute value of the matrix elements. Also note that equation 5.43 is the same as equation B.2 in the case of scalar-valued signals, where $\hat{m}=m=1$.

It can be shown that a unique transfer matrix $\mathbf{H}_{\mathbf{g f}}$ exists for which equation B.1 holds, if and only if

$$
\operatorname{rank}\left[\begin{array}{l}
\mathbf{F} \\
\mathbf{G}
\end{array}\right]=\operatorname{rank} \mathbf{F}=\hat{m}
$$


It follows that $l \geq \hat{m}$. Equation B.4 can be interpreted as the statement that the signal space of $\mathbf{f}$ and $\mathbf{g}$ together is $\hat{m}$ dimensional and the signal space of $\mathbf{f}$ itself is $\hat{m}$ dimensional as well. Combining equations B.3 and B.4, the total least squares problem becomes

$$
\min \left\|\left[\begin{array}{l}
\mathbf{F} \\
\mathbf{G}
\end{array}\right]-\left[\begin{array}{l}
\widetilde{\mathbf{F}} \\
\widetilde{\mathbf{G}}
\end{array}\right]\right\|_{F}^{2} \text { s.t. } \operatorname{rank}\left[\begin{array}{l}
\mathbf{F} \\
\mathbf{G}
\end{array}\right]=\operatorname{rank}[\mathbf{F}]=\hat{m}
$$

There is a theoretical possibility that the minimum does not exist because $\mathbf{F}$ converges to a singular matrix as the distance decreases, such that the constraint that $\operatorname{rank} \mathbf{F}=\hat{m}$ is violated in the limit case. Aside from this theoretical detail, the constraint that $\mathbf{F}$ has full row rank is superfluous and the matrices $\mathbf{F}$ and $\mathbf{G}$ follow from

$$
\min \left\|\left[\begin{array}{l}
\mathbf{F} \\
\mathbf{G}
\end{array}\right]-\left[\begin{array}{c}
\widetilde{\mathbf{F}} \\
\widetilde{\mathbf{G}}
\end{array}\right]\right\|_{F}^{2} \quad \text { s.t. } \operatorname{rank}\left[\begin{array}{l}
\mathbf{F} \\
\mathbf{G}
\end{array}\right]=\hat{m}
$$

Equation B.6 is a well known singular value problem. In practice matrix $\left[\widetilde{\mathbf{F}}^{T}, \widetilde{\mathbf{G}}^{T}\right]^{T}$ has $\hat{m}$ large singular values, which belong to the signal, and $n$ small singular values, which belong to the noise. The SVD can therefore be partitioned as follows.

$$
\left[\begin{array}{c}
\widetilde{\mathbf{F}} \\
\widetilde{\mathbf{G}}
\end{array}\right]=\left[\begin{array}{ll}
\mathbf{U}_{\widetilde{\mathbf{F}} \hat{m}} & \mathbf{U}_{\widetilde{\mathbf{F}} n} \\
\mathbf{U}_{\widetilde{\mathbf{G}} \hat{m}} & \mathbf{U}_{\widetilde{\mathbf{G}} n}
\end{array}\right]\left[\begin{array}{ll}
\boldsymbol{\Sigma}_{\hat{m}} & \\
& \boldsymbol{\Sigma}_{n}
\end{array}\right]\left[\begin{array}{ll}
\mathbf{V}_{\hat{m}} & \mathbf{V}_{n}
\end{array}\right]^{H}
$$

The solution to equation B.6 is found by setting the last $n$ singular values to zero.

$$
\begin{aligned}
\mathbf{F} & =\mathbf{U}_{\widetilde{\mathbf{F}} \hat{m}} \Sigma_{\hat{m}} \mathbf{V}_{\hat{m}}^{H} \\
\mathbf{G} & =\mathbf{U}_{\widetilde{\mathbf{G}} \hat{m}} \boldsymbol{\Sigma}_{\hat{m}} \mathbf{V}_{\hat{m}}^{H} \\
\mathbf{H}_{\mathbf{g f}} & =\mathbf{U}_{\widetilde{\mathbf{G}} \hat{m}} \mathbf{U}_{\widetilde{\mathbf{F}} \hat{m}}^{-1}
\end{aligned}
$$

Equation B.10 can be implemented directly to calculate the transfer function based on experimental data. The same transfer function and singular vectors can also be calculated by applying PCA to the spectral matrix which is estimated by averaging. The estimated spectral matrix and its eigenvalue decomposition are

$$
\begin{aligned}
{\left[\begin{array}{ll}
\widetilde{\mathbf{S}}_{\mathbf{f f}} & \widetilde{\mathbf{S}}_{\mathbf{f g}} \\
\widetilde{\mathbf{S}}_{\mathbf{g f}} & \widetilde{\mathbf{S}}_{\mathbf{g g}}
\end{array}\right] } & =\frac{1}{k}\left[\begin{array}{l}
\widetilde{\mathbf{F}} \\
\widetilde{\mathbf{G}}
\end{array}\right]\left[\begin{array}{c}
\widetilde{\mathbf{F}} \\
\widetilde{\mathbf{G}}
\end{array}\right]^{H} \\
& =\left[\begin{array}{ll}
\mathbf{U}_{\widetilde{\mathbf{F}} \hat{m}} & \mathbf{U}_{\widetilde{\mathbf{F}} n} \\
\mathbf{U}_{\widetilde{\mathbf{G}} \hat{m}} & \mathbf{U}_{\widetilde{\mathbf{G}} n}
\end{array}\right]\left[\begin{array}{ll}
\Sigma_{\hat{m}}^{2} & \Sigma_{n}^{2}
\end{array}\right]\left[\begin{array}{ll}
\mathbf{U}_{\widetilde{\mathbf{F}} \hat{m}} & \mathbf{U}_{\widetilde{\mathbf{F}} n} \\
\mathbf{U}_{\widetilde{\mathbf{G}} \hat{m}} & \mathbf{U}_{\widetilde{\mathbf{G}} n}
\end{array}\right]^{H}
\end{aligned}
$$

As noted in section 5.5.2, the matrices $\mathbf{U}_{\widetilde{\mathbf{F}} \hat{m}}$ and $\mathbf{U}_{\widetilde{\mathbf{G}} \hat{m}}$ can be calculated by applying PCA to a spectral matrix which is calculated by averaging. Furthermore, equation 5.45 is the specific case of equation B.10 where $\hat{m}=m=1$ and the expected value operator is used to arrive at the spectral matrix. 


\section{Appendix C}

\section{Rank of the source matrix}

\section{Introduction}

Section 6.3.2 considers a statement concerning the rank of the source matrix in cross-spectral inverse acoustics. This appendix proves that statement. Firstly, the statement repeated in a more formal way.

Let the source matrix $\mathbf{S}_{\text {st }} \in \mathbb{C}^{n \times \hat{n}}$ be calculated based on the field matrix $\mathbf{S}_{\text {st }} \in$ $\mathbb{C}^{m \times \hat{m}}$ by an equation of the following general form.

$$
\begin{aligned}
\operatorname{vec}\left(\mathbf{S}_{\mathbf{s t}}\right) & =\mathbf{V} \boldsymbol{\Sigma}^{\dagger \alpha} \mathbf{U}^{H} \operatorname{vec}\left(\mathbf{S}_{\mathbf{f g}}\right) \quad \text { where }\left\{\begin{array}{l}
\mathbf{V} \in \mathbb{C}^{m \cdot \hat{m} \times k \cdot \hat{k}} \\
\mathbf{U} \in \mathbb{C}^{n \cdot \hat{n} \times k \cdot \hat{k}} \\
\boldsymbol{\Sigma}^{\dagger \alpha} \in \mathbb{C}^{k \cdot \hat{k} \times k \cdot \hat{k}}
\end{array}\right. \\
k & \leq \min (m, n) \quad ; \quad \hat{k} \leq \min (\hat{m}, \hat{n})
\end{aligned}
$$

Where $\mathbf{V}$ and $\mathbf{U}$ are matrices containing the singular vectors of the Kronecker product of two matrices in accordance with equation 6.25. The matrices are unitary. The matrix $\Sigma^{\dagger \alpha}$ is diagonal and real.

The statement can then be formulated as follows. If equation C.1 yields a source matrix $\mathbf{S}_{\mathbf{s t}}$ of rank $\leq r$ for any field matrix $\mathbf{S}_{\mathbf{f g}}$ of rank $r$, then the inverse calculation can be written in the following form

$$
\mathbf{S}_{\mathbf{s t}}=\mathbf{A} \mathbf{S}_{\mathbf{f g}} \mathbf{B}^{H}
$$

where $\mathbf{A} \in \mathbb{C}^{n \times m}$ and $\mathbf{B} \in \mathbb{C}^{\hat{n} \times \hat{m}}$. This section derives that statement. 


\section{Derivation}

In accordance with equation 6.25 , equation C. 1 can be written as

$$
\begin{aligned}
\operatorname{vec}\left(\mathbf{S}_{\mathbf{s t}}\right) & =\left(\overline{\mathbf{V}}_{\mathbf{g t}} \otimes \mathbf{V}_{\mathbf{f s}}\right) \Sigma^{\dagger \alpha}\left(\overline{\mathbf{U}}_{\mathbf{g t}} \otimes \mathbf{U}_{\mathbf{f s}}\right)^{H} \operatorname{vec}\left(\mathbf{S}_{\mathbf{f g}}\right) \\
\operatorname{vec}\left(\mathbf{V}_{\mathbf{f s}}^{H} \mathbf{S}_{\mathbf{s t}} \mathbf{V}_{\mathbf{g t}}\right) & =\boldsymbol{\Sigma}^{\dagger \alpha} \operatorname{vec}\left(\mathbf{U}_{\mathbf{f s}}^{H} \mathbf{S}_{\mathbf{f g}} \mathbf{U}_{\mathbf{g t}}\right) \\
\operatorname{vec} \widehat{\mathbf{S}} & =\Sigma^{\dagger \alpha} \operatorname{vec} \widehat{\mathbf{F}} \quad \text { where }\left\{\begin{array}{l}
\widehat{\mathbf{S}}=\mathbf{V}_{\mathbf{f s}}^{H} \mathbf{S}_{\mathbf{s t}} \mathbf{V}_{\mathbf{g t}} \\
\widehat{\mathbf{F}}=\mathbf{U}_{\mathbf{f s}}^{H} \mathbf{S}_{\mathbf{f g}} \mathbf{U}_{\mathbf{g t}}
\end{array}\right.
\end{aligned}
$$

The diagonal elements of the diagonal matrix $\Sigma^{\dagger \alpha}$ are rearranged to become the matrix $\widehat{\Sigma}^{\dagger \alpha} \in \mathbb{C}^{k \times \hat{k}}$ such that

$$
\begin{aligned}
\operatorname{vec} \widehat{\boldsymbol{\Sigma}}^{\dagger \alpha} & =\operatorname{diag} \boldsymbol{\Sigma}^{\dagger \alpha} \quad \text { hence } \\
\widehat{\mathbf{S}}_{i j} & =\widehat{\boldsymbol{\Sigma}}_{i j}^{\dagger \alpha} \widehat{\mathbf{F}}_{i j}
\end{aligned}
$$

The rank of the transformed source matrix $\widehat{\mathbf{S}}$ is smaller than or equal to the rank of the transformed field matrix $\widehat{\mathbf{F}}$. Hence, if $\widehat{\mathbf{F}}$ is the arbitrary rank-1 matrix $\widehat{\mathbf{F}}=\mathbf{f g}^{T}$, then $\widehat{\mathbf{S}}$ must have a rank $\leq 1$.

$$
\begin{aligned}
\widehat{\mathbf{S}}_{i j} & =\widehat{\boldsymbol{\Sigma}}_{i j}^{\dagger \alpha} \mathbf{f}_{i} \mathbf{g}_{j} \\
\widehat{\mathbf{S}} & =\operatorname{diag}(\mathbf{f}) \widehat{\boldsymbol{\Sigma}}^{\dagger \alpha} \operatorname{diag}(\mathbf{g})
\end{aligned}
$$

Hence

$$
\operatorname{rank} \mathbf{S} \leq\left\{\begin{array}{l}
\operatorname{rankdiag}(\mathbf{f}) \\
\operatorname{rank} \widehat{\boldsymbol{\Sigma}}^{\dagger \alpha} \\
\operatorname{rank} \operatorname{diag}(\mathbf{g})
\end{array}\right.
$$

Since $\mathbf{S}$ must be rank $\leq 1$ for all rank-1 matrices of the form $\mathbf{F}=\mathbf{f g}^{T}, \widehat{\mathbf{\Sigma}}^{\dagger \alpha}$ must be rank-1 such that it is of the form $\widehat{\boldsymbol{\Sigma}}^{\dagger \alpha}=\mathbf{a b}{ }^{H}$ such that it is rank-1 in general or rank-0 if it is the zero matrix. Hence, returning to equation C.8

$$
\begin{aligned}
\mathbf{S}_{i j} & =\mathbf{a}_{i} \mathbf{b}_{j} \mathbf{F}_{i j} \\
\mathbf{S} & =\operatorname{diag}(\mathbf{a}) \mathbf{F} \operatorname{diag}(\mathbf{b})
\end{aligned}
$$

With equation C.6

$$
\begin{aligned}
\mathbf{V}_{\mathbf{f s}}^{H} \mathbf{S}_{\mathbf{s t}} \mathbf{V}_{\mathbf{g t}} & =\operatorname{diag}(\mathbf{a}) \mathbf{U}_{\mathbf{f s}}^{H} \mathbf{S}_{\mathbf{f g}} \mathbf{U}_{\mathbf{g t}} \operatorname{diag}(\mathbf{b}) \\
\mathbf{S}_{\mathbf{s t}} & =\mathbf{A S}_{\mathbf{f g}} \mathbf{B}^{H} \text { where }\left\{\begin{array}{l}
\mathbf{A}=\mathbf{V}_{\mathbf{f s}} \operatorname{diag}(\mathbf{a}) \mathbf{U}_{\mathbf{f s}}^{H} \\
\mathbf{B}=\mathbf{V}_{\mathbf{g t}} \operatorname{diag}(\mathbf{b}) \mathbf{U}_{\mathbf{g t}}^{H}
\end{array}\right.
\end{aligned}
$$


It is noted that equation C.15 is a consequence of C. 14 only if the rows and columns $\mathbf{S}_{\mathbf{s t}}$ are in the range of $\mathbf{V}_{\mathbf{f s}}$ and $\mathbf{V}_{\mathbf{g t}}$ respectively. This is true because of equation C.4. Equation C.15 and C.3 are the same, which concludes the derivation. 


\section{Appendix D}

\section{DAMAS}

This appendix derives the relation between the problem of equation 6.42 and the DAMAS beamforming method [8]. This is achieved by noting that the singular values of $\mathbf{H}$ can be calculated as the square root of the eigenvalues of $\mathbf{H}^{H} \mathbf{H}$ (see also equation 3.3).

The regularized inverse matrix can be written as

$$
\begin{aligned}
\mathbf{H}^{\dagger \alpha} & =\mathbf{V} \boldsymbol{\Sigma}^{\dagger \alpha} \mathbf{U}^{H} \\
& =\mathbf{V} \boldsymbol{\Sigma}^{\dagger \alpha} \mathbf{U}^{H} \mathbf{H}^{\dagger H} \mathbf{H}^{H} \\
& =\mathbf{V} \boldsymbol{\Sigma}^{\dagger \alpha} \boldsymbol{\Sigma}^{\dagger H} \mathbf{V}^{H} \mathbf{H}^{H}
\end{aligned}
$$

The diagonal matrix $\boldsymbol{\Sigma}^{\dagger \alpha} \boldsymbol{\Sigma}^{\dagger H}$ has the following diagonal elements

$$
\frac{\mathscr{F}_{\alpha}\left(\sigma_{i}\right)}{\sigma_{i}^{2}}=\frac{\mathscr{F}_{\alpha}\left(\sqrt{\sigma_{i}^{2}}\right)}{\sigma_{i}^{2}}
$$

which is a filter applied to the eigenvalues of $\mathbf{H}^{H} \mathbf{H}$. It is denoted as $\left(\mathbf{H}^{H} \mathbf{H}\right)^{\ddagger \alpha}$

$$
\begin{aligned}
\left(\mathbf{H}^{H} \mathbf{H}\right)^{\ddagger \alpha} & =\mathbf{V}\left(\boldsymbol{\Sigma}^{H} \boldsymbol{\Sigma}\right)^{\ddagger \alpha} \mathbf{V}^{H} \\
& =\mathbf{V} \boldsymbol{\Sigma}^{\dagger \alpha} \boldsymbol{\Sigma}^{-1} \mathbf{V}^{H}
\end{aligned}
$$

By applying equation D.5, equation D.3 simplifies to

$$
\begin{aligned}
\mathbf{H}^{\dagger \alpha} & =\left(\mathbf{H}^{H} \mathbf{H}\right)^{\ddagger \alpha} \mathbf{H}^{H} \text { hence } \\
\mathbf{x} & =\left(\mathbf{H}^{H} \mathbf{H}\right)^{\ddagger \alpha} \mathbf{H}^{H} \operatorname{vec}\left(\mathbf{S}_{\mathbf{f f}}\right) \\
& =\mathbf{A}^{\ddagger \alpha} \mathbf{y} \quad ; \quad \begin{cases}\mathbf{y} & =\mathbf{H}^{H} \operatorname{vec}\left(\mathbf{S}_{\mathbf{f f}}\right) \\
\mathbf{A} & =\left(\mathbf{H}^{H} \mathbf{H}\right)\end{cases}
\end{aligned}
$$


where $\mathbf{x}, \mathbf{A}$ and $\mathbf{y}$ are denoted $\hat{X}, \hat{A}$ and $\hat{Y}$ by Brooks [8]. The inverse calculation can be performed by calculating $\mathbf{A}^{\ddagger \alpha}$, which is the filter in equation D.4 applied to the eigenvalues of $\mathbf{A}$. To take into account the fact that the elements of $\mathbf{x}$ are nonnegative, Brooks proposes to use a regularization method of the class of Landweber iteration methods, and to set each negative element to zero at each iteration. The inverse solution can be calculated without storing $\mathbf{H}$ in computer memory. The following two useful relations can be derived by writing the matrix products as summations in the same way as equation 6.40

$$
\begin{aligned}
\mathbf{y} & =\operatorname{diag}\left(\mathbf{H}_{\mathbf{s f}}^{H} \mathbf{S}_{\mathbf{f f}} \mathbf{H}_{\mathbf{s f}}\right) \\
(\mathbf{A})_{i j} & =\left|\mathbf{H}_{\mathbf{s f}}^{H} \mathbf{H}_{\mathbf{s f}}\right|_{i j}^{2}
\end{aligned}
$$

which can be implemented directly. 


\section{Bibliography}

[1] E. Anderson, Z. Bai, C. Bischof, S. Blackford, J. Demmel, J. Dongarra, J. Du Croz, A. Greenbaum, S. Hammarling, A. McKenney, and D. Sorensen. LAPACK Users' Guide. Society for Industrial and Applied Mathematics, Philadelphia, PA, third edition, 1999.

[2] T. G. H. Basten, H.-E. de Bree, W. F. Druyvesteyn, and J. W. Wind. Multiple incoherent sound source localization using a single vector sensor. In Proceedings Sixteenth International Congress on Sound and Vibration, Kraków, Poland, 2009.

[3] L. B. Beasley. Linear operators on matrices: The invariance of rank-k matrices. Linear Algebra and its Applications, 107:161-167, 1988.

[4] Å. Björk. Numerical methods for least squares problems. Society for Industrial and Applied Mathematics, Philadelphia, PA, 1996.

[5] D. T. Blackstock. Fundamentals of Physical Acoustics. Wiley-Interscience, 2000.

[6] H.-E. de Bree and W. F. Druyvesteyn. A particle velocity sensor to measure the sound field from a structure in the presence of background noise. In the proceedings of Forum Acousticum 2005, 2005.

[7] H.-E. de Bree, V. B. Svetovoy, R. Raangs, and R. Visser. The very near field, Theory, simulations and measurements of the sound pressure and particle velocity in the very near field. In the Proceedings Eleventh International Congress on Sound and Vibration, 2004.

[8] T. F. Brooks and W. M. Humphreys Jr. A deconvolution approach for the mapping of acoustic sources (DAMAS) determined from phased microphone arrays. In Proceedings 10th AIAA/CEAS Aeroacoustics Conference, 2004. 
[9] T. F. Brooks and Jr. W. M. Humphreys. Extension of damas phased array processing for spatial coherence determination (damas-c). In AIAA/CEAS Aeroacoustics Conference, pages 3337-3354, Cambridge MA, USA, 2006.

[10] F. O Castres and P F Joseph. Mode detection in turbofan inlets from near field sensor arrays. The Journal of the Acoustical Society of America, 121(2):796807, 2007.

[11] L. Cohen. Time Frequency Analysis. Prentice Hall, 1995.

[12] R. D. Cook, D. S. Malkus, and M. E. Plesha. Concepts and Applications of Finite Element Analysis. John Wiley \& Sons, third edition, 1989.

[13] I. Daubechies. The wavelet transform, time-frequency localisation and signal analysis. IEEE Transactions on Information Theory, 36(5):961-1005, September 1990.

[14] W. F. Druyvesteyn, J. W. Wind, and H. M. Ligtenberg. Near field sound source separation using a single acoustic particle velocity vector sensor. In Proceedings NAG/DAGA, Rotterdam, the Netherlands, 2009.

[15] Y. E. Eldar and A. V. Oppenheim. MMSE whitening and subspace whitening. IEEE Transactions on Iformation Theory, 49(7):1846-1851, 2003.

[16] D. J. Ewins. Modal Testing: Theory, Practice and Application (Mechanical Engineering Research Studies Engineering Design Series). Taylor \& Francis Group, February 2003.

[17] F. J. Fahy. Sound Intensity. Spon Press, London, United Kingdom, second edition, 1989,1995.

[18] M. Fischer, U. Gauger, and L. Gaul. A multipole galerkin boundary element method for acoustics. Engineering Analysis with Boundary Elements, 28(2):155 - 162, 2004.

[19] G. H. Golub and C. F. Van Loan. Matrix Computations. Johns Hopkins University Press, Baltimore, third edition, 1996.

[20] G. H. Golub, F. T. Luk, and M. L. Overton. A block Lanczos method for computing the singular values and corresponding singular vectors of a matrix. ACM Transactions on Mathematical Software, 7:149-169, 1981.

[21] M. Hanke H. W. Engl and A. Neubauer. Regularization of Inverse Problems. Kluwer Academic Publishers, Dordrecht, The Netherlands, 2000. 
[22] J. Hald. STSF - a unique technique for scan-based Near-field Acoustic Holography without restrictions on coherence. Brüel \& Kjær, Technical Review No.1, 1989.

[23] J. Hald. Time domain acoustical holography and its applications. Sound and Vibration, 2:16-25, 2001.

[24] J. Hald. Patch near-field acoustic holography using a new statistically optimal method. In Proceedings Inter-Noise 2003, pages 2203-2210, Jeju Island, Korea, 2003.

[25] J. Hald, J. Mørkholt, J. Gomes, and S. Gade. Efficient interior noise source identification based on conformal mapping using SONAH holography for details on selected panels. In Proceedings Fifteenth International Congress on Sound and Vibration, Daejeon, Korea, 2008.

[26] M. Hanke. On lanczos based methods for the regularization of discrete illposed problems, 2000.

[27] P. C. Hansen. Analysis of discrete ill-posed problems by means of the L-curve. SIAM Journal of Scientific Computing, 34:561-580, 1992.

[28] P. C. Hansen. Rank-Deficient and Discrete Ill-Posed Problems: Numerical Aspects of Linear Inversion. Society for Industrial and Applied Mathematics, Philadelphia (PA), 1998.

[29] P. C. Hansen. Deconvolution and regularization with toeplitz matrices. $\mathrm{Nu}$ merical Algorithms, 29:323-378, 2002.

[30] P. C. Hansen and D. P. O'Leary. The use of the L-curve in the regularization of discrete ill-posed problems. SIAM Journal of Scientific Computing, 14:14871503, 1993.

[31] R. J. Hanson. A numerical method for solving fredholm integral equations of the first kind using singular values. SIAM J. Numer. Anal., 8(3):616-622, 1971.

[32] S. Haykin. Adaptive filter theory. Prentice Hall, second edition, 1991.

[33] F. Jacobsen. A note on instantaneous and time-averaged active and reactive sound intensity. Journal of Sound and Vibration, 147(3):489-496, 1991.

[34] F. Jacobsen and V. Jaud. Statistically optimized near field acoustic holography using an array of pressure-velocity probes. Journal of the Acoustical Society of America, 121(3):1550-1558, mar 2007. 
[35] F. Jacobsen and Y. Liu. Near field acoustic holography with particle velocity transducers. Journal of the Acoustical Society of America, 118(5):3139-3144, nov 2005.

[36] K. Kimitei. Algorithms for Toeplitz Matrices with Applications to Image Deblurring. Master's thesis, Georgia State University, Atlanta GA, USA, 2005.

[37] J. T. King. Multilevel algorithms for ill-posed problems. Numerische Mathematik, 61:311-334, 1992.

[38] L. E. Kinstler. Fundamentals of Acoustics. John Wiley \& Sons, 2000.

[39] V. C. Klema and A. J. Laub. The Singular Value Decomposition: Its Computation and Some Applications. IEEE Transactions on Automatic Control, 25(2):164-176, 1980.

[40] H. Krim and M. Viberg. Two decades of array signal processing research: the parametric approach. Signal Processing Magazine, IEEE, 13(4):67-94, Jul 1996.

[41] H. S. Kwon and Y.-H. Kim. Moving frame technique for planar acoustic holography. Journal of the Acoustical Society of America, 103(4):1734-1741, 1998.

[42] P. K. Lamm. Inverse problems and ill-posedness. In N. Zabaras, K. A. Woodbury, and M. Raynaud, editors, Inverse Problems in Engineering: Theory and Practice, pages 1-10. The American Society of Mechanical Engineers, 1993.

[43] L. de Lathauwer, B. de Moor, and J. Vandewalle. A multilinear singular value decomposition. J. Matrix Anal. Appl., 21(4):1253-1278, 2000.

[44] J. Makhoul. Linear prediction: A tutorial review. Proceedings of the IEEE, 63(4):561-580, 1975.

[45] J. D. Maynard, E. G. Williams, and Y. Lee. Nearfield acoustical holography: I. Theory of generalized holography and the development of NAH. Journal of the Acoustical Society of America, 78(4):1395-1413, 1985.

[46] R. E. Mortensen. Random Signals and Systems. Wiley-Interscience, 1987.

[47] K.-U. Nam and Y.-H. Kim. Visualitzation of multiple incoherent sources by the backward prediction of near-field acoustic holography. Journal of the Acoustical Society of America, 109(5):1808 - 1816, 2001. 
[48] P. A. Nelson and S. H. Yoon. Estimation of acoustic source strength by inverse methods: part I, conditioning of the inverse problem. Journal of Sound and Vibration, 233(4):643-668, 2000.

[49] S. Oerlemans. Detection of aeroacoustic sound sources on aircraft and wind turbines. PhD thesis, University of Twente, Enschede, The Netherlands, 2009.

[50] A. Papoulis. Probability, random variables and stochastic processes. McGrawHill, second edition, 1984.

[51] S. H. Park and Y.-H. Kim. An improved moving frame acoustic holography for coherent bandlimited noise. Journal of the Acoustical Society of America, 104(6):3179-3189, 1998.

[52] S. H. Park and Y.-H. Kim. Effects of the speed of moving noise sources on the sound visualization by means of moving frame acoustic holography. Journal of the Acoustical Society of America, 108(6):2719-2728, 2000.

[53] S. H. Park and Y.-H. Kim. Visualization of pass-by noise by means of moving frame acoustic holography. Journal of the Acoustical Society of America, 110(5):2326-2339, 2001.

[54] A. Paulraj and T. Kailath. Direction of arrival estimation by eigenstructure methods with imperfect spatial coherence of wave fronts. Journal of the Acoustical Society of America, 83(3):1034-1040, 1988.

[55] D. B. Percival and A. T. Walden. Spectral Analysis for physical applications. Cambridge University Press, 1993.

[56] D. B. Percival and A. T Walden. Wavelet Methods for Time Series Analysis. Cambridge University Press, February 2000.

[57] G. Peters and J. H. Wilkinson. $\mathbf{A x}=\lambda \mathbf{B x}$ and the generalized eigenproblem. SIAM Journal on Numerical Analysis, 7(4):479-492, 1970.

[58] B. Picinbono. Random Signals and Systems. Prentice Hall, 1993.

[59] A. D. Pierce. Acoustics, an introduction to its physical principles and applications. Acoustical Society of America, third edition, 1994.

[60] B. Pluymers. Wave based modelling methods for steady-state vibro-acoustics. $\mathrm{PhD}$ thesis, Katholieke Universiteit Leuven, Leuven, Belgium, 2006. 
[61] F. Poisson, J. C. Valiere, and P. Herzog. High speed sound sources localization using bilinear time-frequency transformation. Applied Acoustics, 53(1-3):113, 1998.

[62] M. B. Priestley. Spectral analysis and time series, volume 2:multivariate series. Academic Press, 1981.

[63] M. B. Priestley. Spectral analysis and time series, volume 1: univariate series. Academic Press, 1981.

[64] D. W. Tufts R. J. Vaccaro and G. F Boudreaux-Bartels. Advances in Principal Component Signal Processing, pages 115-146. Elsevier Science Publishers B.V., 1988.

[65] R. Raangs. Exploring the use of the Microflown. PhD thesis, University of Twente, Enschede, The Netherlands, 2005.

[66] I. Hernández Ramírez. Multilevel Multi-Integration Algoritm for Acoustics. $\mathrm{PhD}$ thesis, University of Twente, Enschede, The Netherlands, 2005.

[67] J. W. S. Rayleigh. The Theory of Sound, volume I. Dover publications, second, revised edition, 1945.

[68] J. W. S. Rayleigh. The Theory of Sound, volume II. Dover publications, second, revised edition, 1945.

[69] N. B. Roozen and A. de Boer and C. H.Slump. Inverse acoustics. Project proposal of STW project TWO6618, The Netherlands, 2004.

[70] A. Sarkissian. Extension of measurement surface in near-field acoustic holography. Journal of the Acoustical Society of America, 115:1593-1596, 2004.

[71] A. Sarkissian. Method of superposition applied to patch near-field acoustic holography. Journal of the Acoustical Society of America, 118(2):671-678, 2005.

[72] R. Schmidt. Multiple emitter location and signal parameter estimation. IEEE Transactions on Antennas and Propagation, 34(3):276-280, 1986.

[73] R. Scholte. Fourier Based High-resolution Near-Field Sound Imaging. PhD thesis, Eindhoven University of Technology, Eindhoven, The Netherlands, 2008. 
[74] R. Scholte, I. Lopez, N. B. Roozen, and H. Nijmijer. Truncated aperture extrapolation for fourier-based near-field acoustic holography by means of border-padding. Journal of the Acoustical Society of America, 125(6):3844 3854, 2009.

[75] V. Simonci and D. B. Szyld. Recent computational developments in Krylov subspace methods for linear systems. Numerical Linear Algebra With Applications, 14:1-59, 2007.

[76] D. Slepian. On bandwidth. Proceedings of the IEEE, 64(3):292-300, March 1976.

[77] D. Slepian. Some comments on fourier analysis, uncertainty and modeling. SIAM Review, 25(3):379-393, 1983.

[78] W. Song. Beamforming applied to psychoacoustics - sound source localization based on psychoacoustic attributes and efficient auralization of $3 D$ sound. $\mathrm{PhD}$ thesis, Aalborg University, 2008.

[79] R. Steiner and J. Hald. Near-field acoustical holography without the errors and limitations caused by the use of the DFT. Journal of Acoustics and Vibration, 6, 2001.

[80] A. Tarantola. Inverse Problem Theory and methods for model parameter estimation. Society for Industrial and Applied Mathematics, Philadelphia, PA, 2005.

[81] D. J. Thomson. Spectrum estimation and harmonic analysis. Proceedings of the IEEE, 70(9):1055-1096, 1982.

[82] E. Tijs and H.-E. de Bree. Mapping 3d sound intensity streamlines in a car interior. In Proceedings SAE, Brasil, 2009.

[83] N. Totaro, C. Sandier, and J.-L. Guyader. Identify velocity of a complex source with IPTF method. In Proceedings Fifteenth International Congress on Sound and Vibration, Daejon, South Korea, 2008.

[84] L. N. Trefethen and D. Bau III. Numerical Linear Algebra. Society for Industrial and Applied Mathematics, Philadelphia, PA, 1997.

[85] W. A. Veronesi and J. D. Maynard. Nearfield acoustical holography (NAH) II. Holographic reconstruction algorithms and computer implementation. Journal of the Acoustical Society of America, 81(5):1307-1321, 1987. 
[86] W. A. Veronesi and J. D. Maynard. Digital holographic reconstruction of sources with arbitrarily shaped surfaces. Journal of the Acoustical Society of America, 85:588-598, 1989.

[87] R. Visser. Inverse Source Identification based on Acoustic Particle Velocity Measurements. In Proceedings Internoise, Dearborn MI, USA, 2002.

[88] R. Visser. Acoustic source localization based on pressure and particle velocity measurements. In Proceedings Internoise, pages 665-670, Seogwipo, Korea, 2003.

[89] R. Visser. A Boundary Element Approach to Acoustic Radiation and Source Identification. PhD thesis, University of Twente, Enschede, The Netherlands, 2004.

[90] R. Visser. A Boundary Element Approach to Acoustic Radiation and Source Identification. PhD thesis, University of Twente, Enschede, The Netherlands, 2004.

[91] R. Visser. On the use of higher order and irregularly shaped boundary elements in nearfield acoustical holography. In Proceedings Eleventh International Congress on Sound and Vibration, St. Petersburg, Russia, 2004.

[92] Z. Wang and S. F. Wu. Helmholtz Equation-Least-Squares method for reconstructing the acoustic pressure field. The Journal of the Acoustical Society of America, 102:2020-2032, 1997.

[93] P. R. White, M. H. Tan, and J. K. Hammond. Analysis of the maximum likelihood, total least squares and principal component approaches for frequency response function estimation. Journal of Sound and Vibration, 290:676-689, 2006.

[94] E. G. Williams. Fourier Acoustics, Sound Radiation and Nearfield Acoustical Holography. Academic Press, first edition, 1999.

[95] E. G. Williams. Continuation of acoustic near-fields. Journal of the Acoustical Society of America, 113(3):1273-1281, 2003.

[96] E. G. Williams. Fast fourier transform and singular value decomposition formulations for patch nearfield acoustical holography. Journal of the Acoustical Society of America, 114(3):1322-1333, 2003. 
[97] E. G. Williams, J. D. Maynard, and E. Skudrzyk. Sound source reconstruction using a microphone array. Journal of the Acoustical Society of America, 68(1):340-344, 1980.

[98] E. G. Williams, N. Valdivia, and P. C. Herdic. Volumetric acoustic vector intensity imager. The Journal of the Acoustical Society of America, 120(4):18871897, 2006.

[99] J. W. Wind. Time-frequency analysis for systems without warp drive. Internal document, University of Twente, 2007.

[100] J. W. Wind, M. H. M. Ellenbroek, and A. de Boer. Moving sensors in inverse acoustics. In Proceedings Fifteenth International Congress on Sound and Vibration, Daejon, South Korea, 2008.

[101] J. W. Wind, D. R. Philippens, and A. de Boer. Near source acoustical particle velocity measurements with ambient noise. In Proceedings International Modal Analysis Conference, 2007.

[102] J. W. Wind, E. Tijs, and H.-E de Bree. Source localization using acoustic vector sensors: A MUSIC approach. In Proceedings Novem, Oxford, United Kingdom, 2009.

[103] J. W. Wind, Y. H. Wijnant, and A. de Boer. Fast evaluation of the rayleigh integral and applications to inverse acoustics. In Proceedings Thirteenth International Congress on Sound and Vibration, Vienna, Austria, 2006.

[104] S. H. Yoon and P. A. Nelson. Estimation of acoustic source strength by inverse methods: part II, experimental investigation of methods for choosing regularization parameters. Journal of Sound and Vibration, 233(4):669-705, 2000. 


\section{Nawoord}

Dit proefschrift is niet alleen ontstaan door nadenken, schrijven en herschrijven maar ook door lezen, luisteren en discussiëren met anderen. Juist omdat het echt weleens moeilijk was, ben ik dankbaar dat er de laatste vier en een half jaar altijd mensen in de buurt zijn geweest.

In eerste plaats bedoel ik daarmee natuurlijk de promovendi van TM, want het is fijn geweest om altijd te weten dat er een lunchwandeling is, dat er meer mensen zijn die weleens over een Hilbert ruimte nadenken, en gewoon dat er fijne mensen in de buurt zijn bij wie je altijd langs kunt lopen. Het is vanwege mijn collega's dat ik mijn werk begin 2008 gelijk weer heb opgepakt. Verder bedoel ik daarmee Daniël en Arjan, de afstudeerders die ik begeleid heb. Het is heel boeiend geweest om met hen mee te kijken. Daarnaast onze secretaresse Debbie en alle andere medewerkers en studenten van TM.

Ook wil ik mijn huisgenoten van Calslaan 18 bedanken, want een huis met mensen om me heen, Wikipedia open en meestal een berg zooi, dat is het enige soort thuis dat ik me al die jaren heb gewenst. En natuurlijk mijn familie, die met Laure en Maayke de laatste paar jaar steeds een beetje completer geworden is. De mensen van Pro Deo wil ik ook bedanken. En, in het heel bijzonder, Femke die altijd vlakbij is of ik nu bij haar thuis ben, of in Roemenië zoals op het moment dat ik dit schrijf.

Daarnaast is er een aantal mensen die een directe, inhoudelijke bijdrage aan dit proefschrift hebben geleverd.

- De begeleiding bij het schrijven van het proefschrift is verzorgd door André de Boer en Marcel Ellenbroek van Toegepaste Mechanica en Akoestiek aan de Universiteit Twente.

- De metingen van de harde schijf die gebruikt zijn in hoofdstukken 4, 6 en 7 zijn uitgevoerd in samenwerking met Rick Scholte van de vakgroep Dynamics and Control aan de faculteit werktuigbouwkunde van de Technische Universiteit Eindhoven. 
- Erik Druyvesteyn van de Signals and Systems groep van Univeristeit Twente heeft een specifiek geval van vergelijking 6.21 afgeleid om te laten zien dat inverse akoestiek met kruisspectra een lineair probleem is.

- De afleiding van de DAMAS methode die is opgenomen in appendix D (vergelijkingen D.9 em D.10) ik oorspronkelijk onafhankelijk van het werk van Brooks gemaakt. De relatie met de DAMAS methode van Brooks is uitgewerkt in samenwerking met Pieter Sijtsma van het Nationaal Lucht en Ruimtevaartlaboratorium (NLR).

Graag wil ik mijn dankbaarheid kenbaar maken voor deze en vele andere bijdragen. 